
HYDROCHLORIC ACID AND HYDROFLUORIC AGID
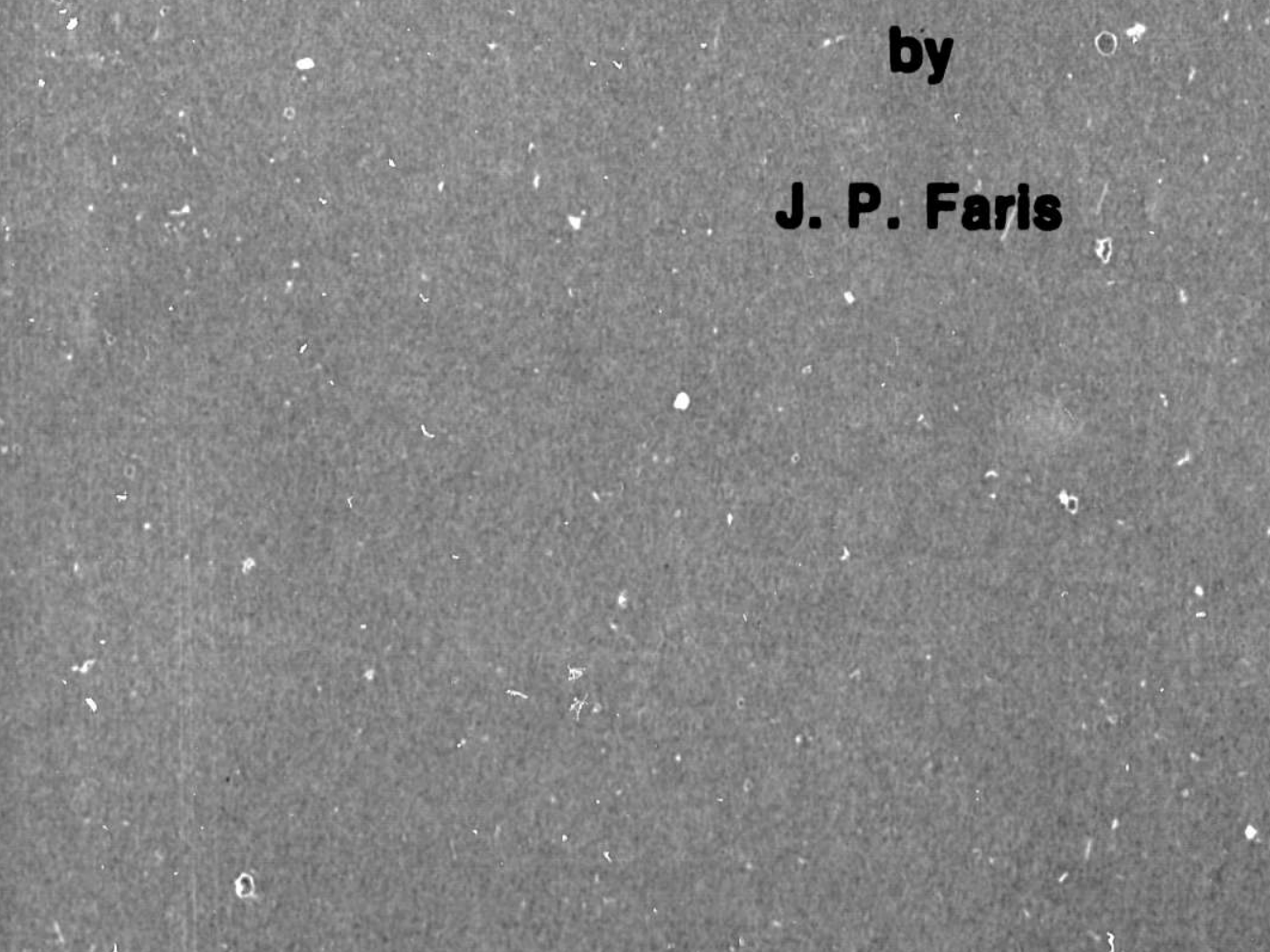

J. P. Faris
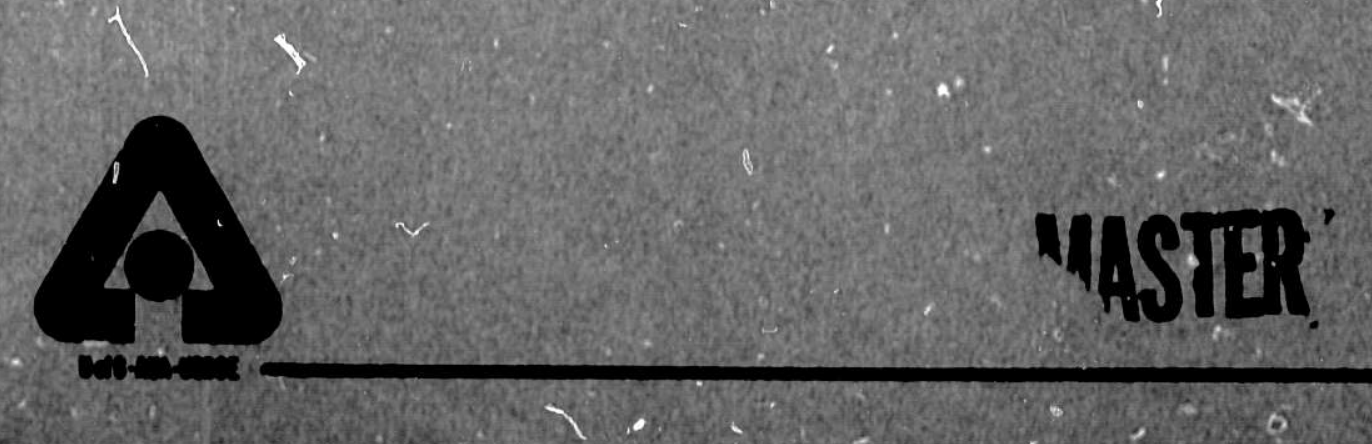

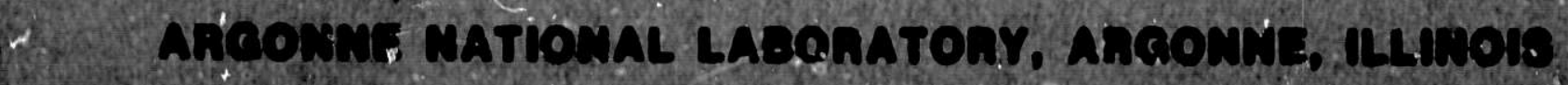

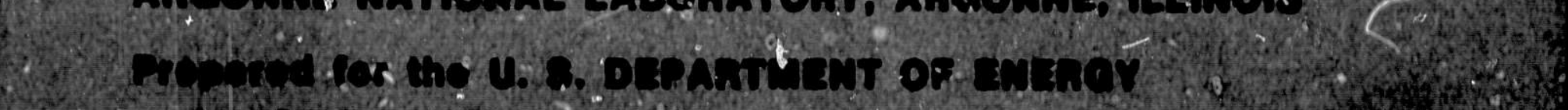

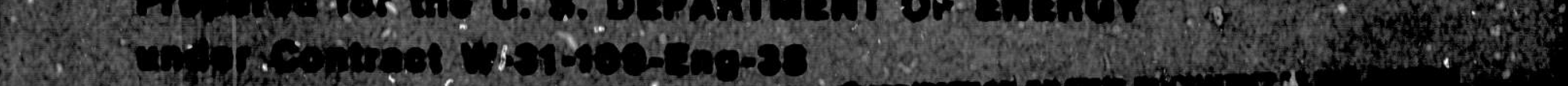


Distribution Category: Chemistry

(UC-4)

ANL-78-78

\begin{abstract}
ARGOINE NATIONAL LABORATORY
9700 South Cass Avenue

Argonne, Illinois 60439
\end{abstract}

SEPARATION OF METAL IONS BY ANION EXCHANGE IN MIXTURES OF HYDROCHLORIC ACID AND HYDROFLUORIC ACID

by

J. P. Faris

Analytica! Chemistry Labcratory

Chemical Engineering Division

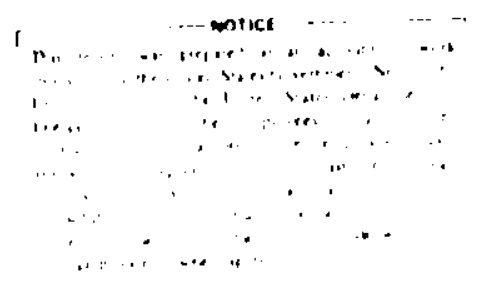

December 1978 

I. ADSORPTION OP METAL IONS ON ANION-EXCHANGE RESINS FROM HCl-HF AND HF , . . . . . . . . . . . . . . 1

A. Introduction ...................... 1

B. Experimental Methud for Determination of Distribution Coefficients . . . . . . . . . . 2

C. Results and Discussion................. 2

II. ANALYTICAL APPLICATIONS OF ANION EXCHANGE

IN THE PRESENCE OF HYDROFLUORIC ACID . . . . . . . . . . . . 3

A. Introduction . . . . . . . . . . . . . . . 3

B. Ion Exchange-Spectrochemical Procedures for

Trace-Element Analysis Using Anion Exchange

in the Presence of Hydrofluoric Acld . . . . . . . . . . 5

1. Analysis of Nlobium for Trace Impurities ......... . 5

2. Procedure for Determination of Niobium, Titanium, Zirconium, Tungaten, and Some Other Blements in Stalnless Steel .................... 6

3. Analysis of Molybdenum for Trace Impurities Using Anton Exchange .................. 6

4. Analysis of Uranium-Plutonium-Molybdenum Alloys for Trace Impuritles . . . . . . . . . . . . 8

5. Anslysis of Cadmium Telluride Semiconductor for Trace Elements . . . . . . . . . . . . . 8

6. Separation Procedure for Trace-Rlement Analysis of Urantum-NLoblum Alloys .............. 9

7. Analyels of $\mathrm{UO}_{2}-10 \% \mathrm{CeO}_{2}$ Alloys for Tungsten . . . . . . . 10

8. Ion-Exchange Procedures for Determination of Trace Elements In High-Purity Aluminum .......... 11

9. Analysis of Aluminum-IIranium Alloys . . . . . . . . . 12

10. Analyols of $80 x$ Aluminum-20x plutonfum Alloy . . . . . 12

REFERBNCES ......................... 13

APPBIDIX. DISTRIBUTION COEFPICIBNTS ROR BLEMANTS IN HC1-BF MIXIURBS, HC1, and HF . . . . . . . . . 15

BIBLIOGRAPEY .......................... 
1. Detection Limits for Ion Exchange-Copper Spark Method ...... . . . . . . . . . . . . 4

2. Aluminum Metal Sample . . . . . . . . . . . . . . 12

A-1. Distribution Coefificients on Dowex $1 \times 10$ AntonExchange Resin (200-400 mesh) as a Function of HC1 and HF Concentrations . . . . . . . . . . . . . .

A-2. Distribution Coefficients on Dowex $1 \times 4$ AntonExchange Resin (100-200 mesh) as a Function of HC1 and HF Concentrations . . . . . . . . . . . . . .

A-3. Distcibution Coefficients on Dowex $1 \times 10$ AntonExchange Resin (200-400 mesh, chloride form) as a Function of HC1 Concentration . . . . . . . . . . . .

A-4. Distribution Coefficients on Dowex $1 \times 4$ AntonBxchange Resin (100-200 mesh, chloride Evrm) as a Function of HCl Concentration ................

A-5. Distribution Coefficients on Dowex 1xiC AnionExchange Resin (100-200 mesh, fluoride form) 88 a Runction of HF Conceatration. . . . . . . . . . . .

A-6. Distribution Coefficients on Dowex 1x4 AnionExchange Resin (100-200 mesh, fluoride form) as a Punction of HE Concentration. ................

A-7. Distribution Coefficients for Several Blements in Dilute Hydrofluoric Acld .................. 


\title{
SEPARATION OR METAL IONS BY ANION EXCHANGE IN MIXTURES OF HYDROCHLORIC ACID AND HYDROFLUORIC ACID
}

J. P. Far1s

\begin{abstract}
Distribution coefficients were determined for the adsorption of more than 40 elements on anion-exchange resins from mintures of HC1 (0.1-12M) and HF (0.1-8M). Two resins, Dowex 1x10, 200-400 mesh and Dowex 1x4, 100-200 mesh, were used. Distribution coeff1clents were also determined for the adsorption of many elements on both resins from $0.1-12 M$ HCl and $0.1-12 M$ HF. Anton exchange in the presence of $\mathrm{HP}$ was found useful for separacing impurities from various materials for their subsequent determination, and specific procedures used in our spectrochemical laboratory for this purpose are out Ined. The results of a 11 terature search on the use of anton exchange in hydrofluoric acid and fluoride-contalning media are presented in an extensive bibilography.
\end{abstract}

\section{ADSORPTION OF METAL IONS ON ANION-EXCHANGE RESINS FROM HC1-HP AND HF}

\section{A. Introduction}

A considerable amount of work has been carried out in the past two or three decades to invest1gate the adsorption of metellic lons on anion-exchange resins from mixtures of hydrochloric and hydrofluoric acids. The early reports of Kraus, Moore, Nelson, and others at Oak Ridge National Leboratury, of Bague, Brown, Bright, and Machlen ac the Nactonal Bureau of Standards and of Wish and others at the U.S. Navel Tadiological Defense Laboratory contained significant Information about the anion-iaxchange behavior of a number of elements in these ac1d mfxtures. Numerous others, Including Dixon and Beadr1dge, de Gells, Farls, Kalinin et al., Nikitin, and Pakholkov et al., have supplied additional deta. inton exchange in HCl-HF mfxtures has been incorporated into separation schemes In many fields of Interest, especially in analytical procedures for isolation of elements where the presence of hydrofluorte acid to desirable to prevent hydrolyeis.

A common problem encountered in our anelytical laboratory 18 the 18olation of trace elemants for their aubsequent determination. This report describes the application of anton exchange in HCl-HF systems to the separation of trace elemants from a wide variety of meterials. An experimental program to wasure dietribution coefficlente of matal lone wap carried out in order to extend the avalleble date on the anton-axchange behavior of elemente from BCl-Bi atxrures as fully as practical. The adsorption of over 40 elements on two anfon-exehange realo, Dowex 1x10, 200-400 mabh and Dowex 1xh, 100-200 wash, we deterined from alxtures which wre 0.1-12N in hydrochloric actd and 0.1-8N In hydrofluoric acid. The extent of adeorption of the eleante from pura hydrochloric actd and from pure hydrofluortc actd was aleo dotexmined uning the can reoins. 
B. Experimental Method for Determination of Distribution Coefficients

The colum-elution technique was employed, utilizing for the most part a series of $3 / 16$ ID polyethylene columins. A typical colum contained $0.5 \mathrm{~g}$ of anion-exchange resin in the chloride form (oven $\mathrm{dried}$ at $\sim 100^{\circ} \mathrm{C}$ ) supported by a plug of plastic wool near the bottom tip. The experiments in pure hydrofluorlc acid were carried out with the resin in the fluorlde form. After a column was equilibrated with the proper acid or acid mixture, microgram amounts of a convenient number of elements, usually from 5 to 8 , were placed In a small volume $(20.2 \mathrm{~mL})$ of the appropriate solution and allowed to flow onto a column. Elution was continued and effluents were collected in fractions of $5 \mathrm{ml}$ for analysis. A continuous elution procedure was used, adding a new group of elements as was conventent. Two sets of stock solutions of Individual elements were prepared. One set contained the elements dissolved in HCl; one set contalned the elements dissolved In HF. Portions of these solutions were comb ined to prepare appropriate stock solutions as needed. Particular care was taken to completely exclude all traces of chloride from the hydrofluoric acid solutions. The effluent fractions were spectrochemically analyzed by the copper spark method.1,2 An aliquot of each effluent fraction was evaporated on the flat ends of copper electrode pairs, sparked under standard conditions, and the spectra photographed in the appropriate wavelength regions. The amount of each element in an effluent fraction was visually estimated by comparing line Intensities with those of a standard plate. Bstimations could be made rapidly and with sufficient accuracy to permit close approximations of elution-curve maxima. Weight distribution coefficients were obtained using the relation $D=(V-1) / M$, where $V$ is the volume in milliliters that had passed through a column when the elution curve maximum was reached, 1 is the first colum volume of interstitial liquid, and $M$ is the mass of resin in grams.

Determination of distribution coefficients of the more atrongly adsorbed elements is impractical using the column-elution technique. Some of the values reported here were estimated by passing relatively large volumes through a colum, but generally the elution was terminated after collecting 30-60 fractIons, and elements that did not break through were reported as "adsorbed." Preliminary experiments ${ }^{3}$ demonstrated that quantitative spectrochemical analysis could be employed to estlmate the adsorption of an element after batchwise equilibration. This procedure, however, did not offer any speclal advantage since radlochemical or other methods of analyals would generally be preferred for more accurate measurements of values for the wore strongly adsorbed lons.

\section{Results and Discussion}

The experimental values for distribution coefficlents are presented in Tables A-1 through A-7 in the Append1x. In Tables A-1 and A-2, weight distribution coefficients are given for the elements in mixtures of BCI (0.1-12M) and IF (0.1-8M) using Dowex 1x10, 206-400 mesh resin and Dowex 1x4, 100-200 mesh resin, respectively. Tables $A-3$ and $A-4$ give the respective results for the adsorption of elements in pure hydrochloric acid $(0.1-12 M)$ for the two resins, and Tables $A-5$ and $A-6$ give the respective results in pure hydrofluoric acid (0.1-12N) for the two resins. Additional experiments were run to determine the adsorption of some elements at lower HP concentrations, and these data are reported in Table A-7. The numbers marked with an asterisk ars aselgned values, extimated from curves drawn using experimental $D$ values found at other acid 
concentrations. The distribution coeff' lent of an element in any acid mixture within the experimental range may be similarly estimated.

The values presented in the tables were, as a rule, in agreement with literature values that are generally accepted as reliable. Blements tended to be more strongly adsorbed on the Dowex $1 \times 10$ resin than on the Dowex $1 \times 4$ resin, which has less cross-1inkage.* Values are not given in the tables for the alkall and alkaline earth elements; they are not adsorbed from elther acid and would not be expected to be adsorbed from a mixture.

The results orlginally published by this laboratory on the adsorption of elements from hydrofluoric acid ${ }^{4}$ have been refined for many elements and extended to $0.1 \mathrm{M}$ acidity. It was found that the strong adsorption of mercury(II) reported in the previous work was in error, probably due to traces of chloride in the stock solution.

\section{ANALYTICAL APPLICATIONS OF ANION EXCHANGE IN THE PRESENCE OF HYDROFLUORIC ACID}

\section{A. Introduction}

Anion exchange in fluoride-containing media has been applied to separations ranging from the macro level in the quantitative analysis of high-temperature alloys to the trace level after neutron activation of high-purity materials. Numerous separation schemes have been published which include anion exchange in the presence of hydrofluoric acid. An examination of the bibliography will 11lustrate the scope of applications.

The work of our spectrochemical laboratory in this area has been directed to the separation of trace elements for their determination in nuclear reactor materials and high-purity metals. Ion exchange is a convenient way to separate metal impurities for spectrochemical analysis since analytical determination may readily be performed at the parts-per-million level without the task of preparing standards for each type of matrix. Knowledge of the Ion-exchange behavior of any elements permts the analyst to select an optimim procedure for efficlent separation of the elements desired. For example, Buff ${ }^{5}$ used anton exchange in nitric-hydrofluoric acid to improve the procedure for determination of trace impurities in tanialum.

Some examples of procedures used in our spectrochemical laboratory are outlined below. Samples with known amounts of Impuritles added were frequently employed to test the efficlency of a procedure. High-purity reagents were generally used, and a control column was carried along to determine the "blank" level for the most common impurities. The nominal detection 1 imits for elements (Table 1) were obtained using a Baird three-meter grating spectrograph.

*iotribution coefficients for elements on the Dowex $1 \times 8$ resin may be estimated from the values obtained on the Dowex $1 \times 10$ and Dowex $1 \times 4$ resins. 
Table 1. Detection Limits ${ }^{a}$ for Ion Exchange-Copper Spark Method

\begin{tabular}{|c|c|c|c|c|c|}
\hline \multirow[b]{2}{*}{ Element } & \multicolumn{2}{|c|}{$\begin{array}{l}\text { Detection Iimit, } \\
\text { ppm }\end{array}$} & \multirow[b]{2}{*}{ Element } & \multicolumn{2}{|c|}{$\begin{array}{c}\text { Detection Limit, } \\
\mathrm{ppm}\end{array}$} \\
\hline & $\begin{array}{l}\text { 100-mg } \\
\text { Sample }\end{array}$ & $\begin{array}{l}1-g \\
\text { Sample }\end{array}$ & & $\begin{array}{l}100-m g \\
\text { Sample }\end{array}$ & $\begin{array}{l}1-8 \\
\text { Sample }\end{array}$ \\
\hline 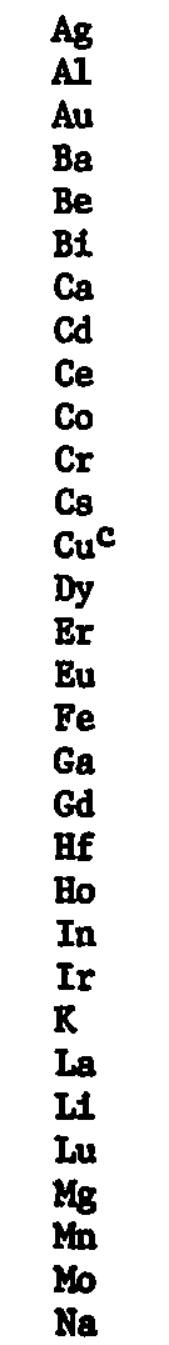 & $\begin{array}{c}2 \\
1^{\mathrm{b}} \\
4 \\
1 \\
0.01 \\
3 \\
1^{\mathrm{b}} \\
4 \\
4 \\
2 \\
2 \\
40 \\
5 \\
2 \\
1 \\
0.2 \\
10 \\
1 \\
2 \\
3 \\
2 \\
3 \\
10 \\
1 \\
0.2 \\
0.01 \\
0.1 \\
1^{\mathrm{b}} \\
0.4 \\
0.5 \\
1 \mathrm{~b}\end{array}$ & $\begin{array}{l}0.2 \\
0.1^{\mathrm{b}} \\
0.4 \\
0.1 \\
0.001 \\
0.3 \\
0.1^{\mathrm{b}} \\
0.4 \\
0.4 \\
0.2 \\
0.2 \\
4 \\
0.5 \\
0.2 \\
0.1 \\
0.02 \\
1 \\
0.1 \\
0.2 \\
0.3 \\
0.2 \\
0.3 \\
1 \\
0.1 \\
0.02 \\
0.001 \\
0.01 \\
0.1 \mathrm{~b} \\
0.04 \\
0.05 \\
0.1 \mathrm{~b}\end{array}$ & 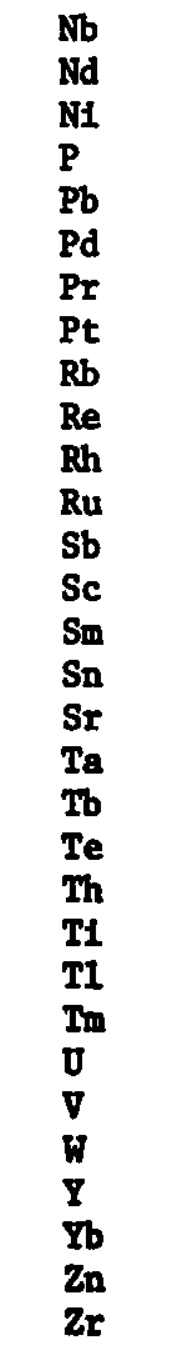 & $\begin{array}{c}4 \\
3 \\
2 \\
50 \\
4 \\
1 \\
4 \\
10 \\
3 \\
10 \\
1 \\
1 \\
40 \\
0.1 \\
5 \\
5 \\
0.02 \\
5 \\
10 \\
50 \\
8 \\
1 \\
10 \\
2 \\
40 \\
1 \\
5 \\
0.05 \\
0.1 \\
10 \\
1 \\
1\end{array}$ & $\begin{array}{l}0.4 \\
0.3 \\
0.2 \\
5 \\
0.4 \\
0.1 \\
0.4 \\
1 \\
0.3 \\
1 \\
0.1 \\
0.1 \\
4 \\
0.01 \\
0.5 \\
0.5 \\
0.002 \\
0.5 \\
1 \\
5 \\
0.8 \\
0.1 \\
1 \\
0.2 \\
4 \\
0.1 \\
0.5 \\
0.005 \\
0.01 \\
1 \\
0.1\end{array}$ \\
\hline
\end{tabular}


B. Ion Exchange-Spectrochemical Procedures for Trace-Element Analysis Using Anion Exchange in the Presence of Hydrofluoric Acid

1. Analysis of Nioblum for Trace Impurities

(J. P. Far18)

a. Experimental Procedure

Place a welghed $100-200 \mathrm{mg}$ sample of nioblum in a Teflon beaker and dissolve in $\mathrm{BNO}_{3} \mathrm{HF}$. Evaporate to dryness, add $\mathrm{HF}$ and repeat, then take up in 10-20 mL of $0.2 \mathrm{MF}$. Add sample solution to a preconditioned antonexchange colum containing resin in the fluoride form. A plastic colum, $1 / 2$ in. ID, filled with Dowex $1 \times 8$ to $a$ helght of about 4 in. 18 satisfactory. Elute Impuritles as outlined below.

(1) Wash with about $50 \mathrm{~mL}$ of $0.2 \mathrm{M} \mathrm{HF}$ to elute the non-adsorbed elements ( $\mathrm{Cd}, \mathrm{Zn}, \mathrm{Co}, \mathrm{NI}, \mathrm{Cr}$, etc.). Wash with an additional $25 \mathrm{~mL}$ of $0.5 \mathrm{M} \mathrm{HF}$ to elute $\mathrm{BI}$.

(2) Wash with about $40 \mathrm{~mL}$ of $0.15 \mathrm{M} \mathrm{HC1-0.2M} \mathrm{HF}$ to ensure elution of $\mathrm{Al}, \mathrm{Be}, \mathrm{Fe}, \mathrm{In}, \mathrm{V}, \mathrm{U}, \mathrm{Te}$, and $\mathrm{Ga}$. This may be combined with eluate (1) above.

(3) Wash with about $75 \mathrm{~mL}$ of $5 M \mathrm{HCl}-8 M \mathrm{HF} .{ }^{*}$ This elutes Mo, $W, \mathrm{Zr}, \mathrm{Hf}$, and $\mathrm{Ti}$.

(4) Wash with about $60 \mathrm{~mL}$ of $2 M \mathrm{HCl}-8 M \mathrm{HF}$ to elute $\mathrm{Sn} .+$

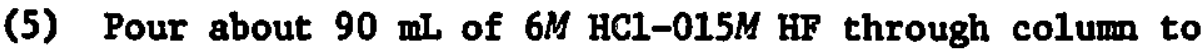
elute Nb matrix. Discard.

(6) Wash with about $75 \mathrm{~mL}$ of $12 M \mathrm{mNO}_{3}-5 M \mathrm{HF}$ to elute Ta. $\dagger$

(7) Evaporate the effluent solutions containfig the Impurities to a volume of $1 \mathrm{mil}$ and determine the metals present by the copper spark method, 1,2

\section{b. Alternative Procedure for Analyels of Mobium** \\ (B. A. Huff)}

Dissolve sample as above and take up In a $7 M$ BC1-4M BF acld mixture. Add sample to an anton-exchange colum which has been preconditioned with this mixture.

(1) Wash with about $60 \mathrm{~mL}$ of $7 \mathrm{H}$ BCl-4M HF. This alutes Al, Be, $\mathrm{Ca}, \mathrm{Cr}, \mathrm{Bf}, \mathrm{Km}, \mathrm{N1}, \mathrm{Pb}, \mathrm{T}, \mathrm{V}, \mathrm{W}, \mathrm{Zr}$, and other non-adsorbed alements.

\footnotetext{
"Acid mixtures are described by the actual, final concentration of each component in the mixture.

tElution of $8 \mathrm{n}$ may be Incomplete. Thls step way be owlted whenever the deternination of $8 \mathrm{a}$ is not required. 
(2) Wash with about $40 \mathrm{~mL}$ of $3 M \mathrm{HCl}-8 M \mathrm{HF}$. This elutes Co, $\mathrm{Fe}, \mathrm{Ga}, \mathrm{Mo}$, and $\mathrm{U}$.

(3) Proceed as in steps 5, 6, and 7 in B.1.a above to obtain Ta and complete analysis.

2. Procedure for Determination of Niobium, Titanium, Zirconium, Tungsten, and Some Other Elements in Stainless Steel

(J. P. Far18)

Weigh 100 mg of sample and dissolve in HCl-HF mixture using a Teflon beaker. Evaporate to inciplent dryness and take up in about $10 \mathrm{~mL}$ of $0.1 M$ $\mathrm{HCl}-0.5 \mathrm{M} \mathrm{HF}$. Pour through a small preconditioned anion-exchange column. A Blo-Rad plastic column $(0.7 \times 10 \mathrm{~cm})$ filled with Dowex $1 \times 8,100-200$ mesh resin Is satisfactory.

(1) Wash well with the $0.1 \mathrm{M} \mathrm{HC1-0.5M}$ HF mixture to remove $\mathrm{Fe}, \mathrm{Cr}$, $\mathrm{NI}, \mathrm{Mn}$, etc. and discard washings. Under these conditions, $\mathrm{Nb}$, Mo, $W, \mathrm{Zr}$, Hf, $\mathrm{TI}, \mathrm{Sn}$, and $\mathrm{Ta}$ remain on the column.

(2) To elute the $\mathrm{Zr}, \mathrm{Hf}, \mathrm{T1}, \mathrm{Nb}$, and $\mathrm{W}$, wash with $6 \mathrm{M} \mathrm{HC1-0.15M} \mathrm{HF}$ unt 11 about $20 \mathrm{~mL}$ have been collected.

(3) Evaporate to a small volume (1 mL) and determine the elements present by the copper spark method. 1,2

The Mo, $\mathrm{Sn}$, and $\mathrm{Ta}$ remalning on the column may be recovered for analysis by washing the column with a strong nitric acid-hydrofluoric acid mixture. Elution of Mo and $\mathrm{Sn}$ may be completed with about $20 \mathrm{~mL}$ of $4 \mathrm{M} \mathrm{HNO}_{3}-4 \mathrm{M} \mathrm{HF}$ mixture; Ta may be eluted with a similar amount of $12 \mathrm{M} \mathrm{HNO}_{3}-5 \mathrm{M} \mathrm{HF}$. Evaporate the solutions to dryness to remove any free nitric acld, take up in $1 \mathrm{~mL}$ of $\mathrm{HCl}-\mathrm{HF}$ solution and determine the elements present by the copper spark method.

3. Analysis of Molybdenum for Trace Impurtt1es Using Anion Exchenge (J. P. Paris)

The determination of trace impurities in molybdenum metal 18 not readily accomplished by direct spectrochemical analyois. The published methods are not practical for occasional samples because they may require installation of apecialized equipment as well as the lengthy task of preparing adequate standards. Furthermore, no single method is applicable for the detection of every element that could likely be present in high-purity material. An anionexchange separation procedure has been developed to 1solate a number of Impurfties and permit their determinațion at the parts-per-milition level by the copper spark method. Good agreement of results was obtained on one sample that was also anelyzed by spark-80urce mass spectroscopy. The procedure 18 presented below:

\section{Procedure for Fluoride-Soluble Blemente}

(1) Weigh 100-300 ing semple, plece in Teflon beaker and diseolve In distilled $7 W$ nitric acid. Bvaporete and convert to an BCl-HF medium by taking to drynese severel times, adding 
HC1 plus a small amount of HF. Take up the final residue In $215 \mathrm{~mL}$ of $6 M \mathrm{HCl}-0.1 M$ HF mixture.

(2) Add the sample solution to a preconditioned anion-exchange column. A plast1c colum, 1/2 In. ID, containing about 4 in. Dowex 1x4, 100-200 mesh resin, is sultable. Wash with $6 M$ HCl-0.1M HF until about $100 \mathrm{~mL}$ of effluent 18 collected. This fraction (A) contains $\mathrm{Nb}, \mathrm{Zr}$, Hf, (W), * $\mathrm{Pb},(\mathrm{In}), \mathrm{V}, \mathrm{As},+(\mathrm{Co}), \mathrm{Al}, \mathrm{Be}, \mathrm{Cr}, \mathrm{Mn}, \mathrm{N} 1$, and other non-adsorbed elements.

(3) Wash with $225 \mathrm{~mL} 6 M \mathrm{HCl}-0.7 M \mathrm{HF}$ to ensure complete elution of $W$. This fraction (B) contains $W$, (Co), (In). It may be combined with fraction A for analysis.

(4) Wash with $100 \mathrm{ml} 1 M \mathrm{HF}$. This fraction (C) contains Co, $\mathrm{Bi}, \mathrm{In},(\mathrm{Fe}), \mathrm{Ga}, \mathrm{Cd}, \mathrm{Zn},(\mathrm{U})$.

(5) Wash with $50 \mathrm{~mL}$ of $0.15 \mathrm{M} \mathrm{HCl}-0.2 \mathrm{MFF}$. This fraction (D) contains $\mathrm{Te}$ ar.: any remaining $\mathrm{U}, \mathrm{Fe}$.

(6) Remove molybden ic $[i$. column with about $200 \mathrm{~mL} 1 M$ HCl."

(7) Wash with $275 \mathrm{~mL} 12 \mathrm{x}: \mathrm{aNO}_{3}-5 \mathrm{M}$ HF. Th1s fraction (E) contains $\mathrm{Ta}, \mathrm{Sn}, \mathrm{Pd}, \mathrm{Re}{ }^{\dagger}$

(8) The various 1mpurity fractions are evaporated to inciplent dryness and examined visually. If no appreciable residue is observed, ${ }^{* *}$ fractions $A$ and $B$ and fractions $C$ and $D$ may be combined for analysis. Dissolve in HC1-HF and adfust volume of each sample to $1 \mathrm{~mL}$. Determine metals present by the copper spark method.1,2

b. Procedure for Determination of the Rare Earths and Thorlum

Weigh a separate portion of sample and proceed as described in steps 1 and 2 of B.3.a above but use 6 to $7 \mathrm{M} \mathrm{HCl}$ solution, omitting the HF. The effluent $A$ will contain the rare-earth elements, Th, and other non-adsorbed metal 1ons. Evaporate to $1 \mathrm{~mL}$ and analyze by the copper spark method.1,2

\footnotetext{
*Elements enclosed in parentheses may not be completely eluted.

tLoses of As, Re, and poselbly Sn may occur by volatilization. Recoveries of $\mathrm{Sn}_{\mathrm{n}} \mathrm{Pd}$, and $\mathrm{Re}$ have not been verified.

中

Th1s part of the procedure needs further work, beceuse an appreciable anount of molybdenum sometimes remains on the colum and 18 eluted with fraction $B$. A second pass through the colum 18 then required. ** Any mafor Impurity or breakthrough of matrix would greatly raduce detection 1infte.
} 
4. Analysis of Uranium-Plutonium-Molybdenum Al.1oys for Trace Impurities (E. A. Huff and J. P. Faris)

Analysis of uranium-plutontum-molybdenum alloys has previously been carried out by determining the impurities in the presence of molybdenum after separating the uranium and plutonium. The amount of molybdenum present (2-4\%) limited the sample aliquot that could be taken for copper spark analysis. The previously described procedure which was developed for the analysis of molybdenum was subsequently applied to the analysis of these alloys to 1mprove the detection 1imits for trace impurities as outlined below.

Procedure:

(1) Dissolve a weighed sample, usually $100-300 \mathrm{mg}$, in $8 M$ nitric ac1d and pass through a dual anton exchange-partition chromatography coluniz to remove $U$ and $\mathrm{Pu}$, as described previously. ${ }^{7}, 7 \mathrm{a}$ Plutonium is alinost selectively retained by an anfon exchange resin and uraniuin is extracted from solution by diethylhexy1ethylhexyl phosphonate fixed on plastic powder.

(2) Evaporate the effluent, which contains Mo and Impurities, and convert to an HCl system by taking to dryness several times with HCl. Take up the final residue in $6 M$ HCl and pass through a preconditioned anion-exchange colum in the chloride form. A 1/2-1a. plastic column containing approximately $5 \mathrm{in}$. of Dowex 1x4, 100-200 mesh is sultable. Wash with 60-80 mL $6 M$ HCl and evaporate effluent to $1 \mathrm{~mL}$ for analysis. This fraction contains $\mathrm{Na}, \mathrm{Lf}, \mathrm{K}, \mathrm{Rb}, \mathrm{Cs}, \mathrm{Ba}, \mathrm{Sr}, \mathrm{Ca}, \mathrm{Mg}, \mathrm{B}, \mathrm{N1},(\mathrm{Co}), \mathrm{Cr}$, $\mathrm{Mn}, \mathrm{Cu}, \mathrm{V}, \mathrm{Al}, \mathrm{As}, \mathrm{Pb}, \mathrm{TI},(\mathrm{In}),\left(\mathrm{Ag}_{\mathrm{g}}\right.$ ?), $\mathrm{Am}$, and rare-earth elements.

(3) Wash column with 80-100 mL $1 M$ HF. This fraction contains Cd, $\mathrm{Zn}, \mathrm{Ga}, \mathrm{BI},(\mathrm{Pe})$ and any remaining $\mathrm{Co}$ and $\mathrm{In}$.

(4) Wash colum with 230 mL $0.15 M$ HC1-0.2M HF to ensure complete elution of $\mathrm{Fe}$ and $\mathrm{Te}$. Combine with previous HF fraction from step 3 and evaporate to a small volume for analysis by the copper-spark method. 1,2

\section{Analyals of Cadmium Telluride Semiconductor for Trace Elements (J. P. Faris)}

Direct analysis of cadmium telluride samples using a direct current (dc) arc gave falrly good detection limita for many of the common elements. To determine the rare earths and to increase the sensitivity for a number of other elements, the anton-exchange procedure outlined below was found to be effective. Determination of many elements could be made in the fractional parts-per-million range.

\section{Procedura:}

(1) D1880." e a welghed 100-5C0 mg eample in aqua regia, evaporate to dryness and take up in $3 \mathrm{H} \mathrm{BCl}$ ( $(15 \mathrm{~mL})$. 
(2) Add the sample solution to a preconditiotisd anton-exchange colum. A plastic 1/2-1n. colum, containing about $4 \mathrm{in}$. Dowex 1x4, 100-200 mesh resin, is convenient. Wash with about $75 \mathrm{~mL} 3 \mathrm{H}$ HCI. The rare-earth elements and most common 1mpurities are eluted while the matrix material (cadmium and tellurium) is retained on the resin.

(3) Elute Te with 50-60 mL $1 M$ HC1-0.1M HF mixture. Discard.

(4) Elute Mo and Sn with 2100 ml of $2 M$ HCl- $8 M$ HF mixture.

(5) Elute $\mathrm{Zn}$ with $250 \mathrm{~mL}$ of $0.1 \mathrm{BCl}$.

(6) Evaporate the three Impurity solutions to a small volume (1 mL) and determine the elements present by the copper spark method.1,2

A control column should be carried along using the same procedure to determine the "blank" level for the most common Impurities.

6. Separation Procedure for Trace-Element Analysis of Uranium-Nioblum Alloys (J. P. Faris)

The carrier-distillation method 8 is routinely used in the spectrochemical analysis of high-purity uranium-nioblum alloys. This method is effective for many impurities but may not include some of the more refractory elements (i.e., Ta, $W, \mathrm{Zr}$ ). A two-step separation procedure designed primarily for the determination of this group is outlined below. * Th1s procedure also allows the determination of a number the elements normally found by the dc arc technique and is useful for verification or cross-checking of results. The nioblum content of the samples ranged up to $30 \%$, but analygis of alloys of any uranium-niobium ratio could be made with only glight modification in the size of the Ion-exchange column.

Procedure:

(1) Place a weighed sample, $200 \mathrm{mg}$, In a Teflon beaker and dissolve In $8 M$ nitric acid plus a small amount of hydrofluoric acid. Remove uranfum by partition chromatography, as described previously., 78 Diethylhexylethylhexyl phosphonate was used as the extractant because of 1 ts good stability 1 . the presence of hydrofluoric acid.

(2) Bvaporate the effluent containing the niobium plus impurities to dryness several times after dissolving in BCl-BR. Binelly diseolve in $\mathrm{U15}$ m of $0.1 \mathrm{H}$ BCI-0.5N BF solution. Pass through a praconditfoned anton-axxhange column and wash the resin with 260 in of $0.1 \mathrm{~W}$ BCI-0.5N HF. A plestic colum, 1/2 In. ID containing about 4 in. Dowex 1x8, 100-200 mesh resin is sultable. The effluent contains the cownon ispurities that are not adsorbed from this acid mixture. 
(3) Wash the resin column with $050 \mathrm{~mL}$ of $6 M$ HC1-6M HF solution. The effluent contains $\mathrm{Zr}$, Hf, $\mathrm{TI}, \mathrm{W},(\mathrm{Mo}),{ }^{*}(\mathrm{Re})$ and any remaining V. Possibly some $\mathrm{Pd}, \mathrm{Be}$ or $\mathrm{Cd}$ may break through.

(4) Wash the column wich $\sim 75$ mL of $7 M$ HCl $-0.2 M$ HF to remove nioblum. Discard.

(5) Wash columm with $60 \mathrm{~mL}$ of $12 \mathrm{M}$ HNO $_{3}-5 \mathrm{M}$ HF to elute $\mathrm{Ta}, \mathrm{Sn}, \mathrm{Be}$, $\mathrm{Cd}, \mathrm{Pd}$, Mo and Re. (Results for the latter four elements lacked good reproduclbility; further checking is needed.)

(6) Evaporate affluents obtained in steps 2, 3, and 5 and take each up in 1 wL of HCl-HF solution. Determine elements present by the copper spark method.1,2

\section{Analygis of $\mathrm{UO}_{2}-10 \% \mathrm{CeO}_{2}$ Alloys for Tungeten (J. P. Faris)}

A procedure used for the analysis of uranium oxide-10\% certum oxide is outlined below. Matrix elements (uranium and cerfum) were removed by precipitation and anion exchange, after which tungsten was 1solated for spectrochemical determination at the parts-per-million level. Other impurities which could also be determined using this procedure are $\mathrm{TI}, \mathrm{Zr}, \mathrm{Hf},(\mathrm{Nb}), \mathrm{V}, \mathrm{Al}, \mathrm{Be}$, and $\mathrm{N1}$, as well as other elements that are soluble in $\mathrm{HF}$ and are not adsorbed by an anton-exchange resin under the conditions used.

Procedure:

(1) Weigh sample, place in Teflon beaker, and dissolve in $27 \mathrm{M} \mathrm{HNO}_{3}$ plus a few drops of HF. A sample weight of 200-400 $\mathrm{mg}$ is preferred.

(2) After dissolution, add to 2-5 mL concentrated HF to precipitate $\mathrm{CeF}_{3}$ and to dissolve the tungsten. Digest for one-half hour or longer, breaking up the larger particles with a plastic rod.

(3) Evaporate to less than $10 \mathrm{~mL}$, transfer to a plastic vial and adjust the volume to $10-15 \mathrm{~mL}$ with water. Mix, allow precipitate to settle, and decant an allquot, usually about one-half of the sample solution, into a Teflon beaker. Evaporate to dryness and dissolve in $7.7 \mathrm{M}$ HCl-3M HF.

(4) Prepare an anion-exchange column and precondition the resin with the 7.7M HC1-3M HF mixture. A plastic colum, 1/2 in. ID, containing about 3 or $4 \mathrm{in}$. Dowex 1x8, 100-200 mesh 18 suitable. Add the sample solution to the colum and wash with $7.7 \mathrm{M}$ HCl-3M HF collecting about $75 \mathrm{~mL}$ of the effluent. Evaporate to a $8 \mathrm{mall}$ volume ( $1 \mathrm{~mL}$ ) and analyze by the copper epark method.1,2

Hote: For some samples an add1t1onal $50 \mathrm{~mL}$ of wash was collected and analyzed to check for completeness of tungsten elution. Since none was detected In any of these fractions, It was assumed that the tungsten recovery was quantitative in the firat fraction. 
This procefure could ale. be modified to include determination of niobium by first washing the coluri. Ath about $50 \mathrm{~mL}$ of $6 \mathrm{M}$ HCl-0.1M HF to elute $\mathrm{Nb}$, then continuing as above.

\section{Ion-Exchange Frocedures for Determination of Trace Elements in High-Purity Aluminum}

Direct spectrochemical analysis of aluminum metal for most trace Impurities is readily accomplished using the dc arc. However, analysis for additional impurities may be carried out using Ion-exchange procedures to separate the elements of interest for their determination. The procedures described below take advantage of the fact that aluminum is not retained by either anion or cation exchange resins from dilute HC1-HF solutions.

Procedures:

Welgh two sample portions of 100-500 mg each, depending upon detection limits desired. Dissolve in distilled $6 \mathrm{M} \mathrm{HCl}$ plus a stall amount of HF, eveporate to inciplent dryness and dissolve in $0.15 \mathrm{M} \mathrm{HCl}-0.2 \mathrm{M} \mathrm{HF}$.

a. Anion Exchange

(1) Pass the solution through a small preconditioned anionexchange column. A plastic colum, $0.7 \mathrm{~cm} \times 4 \mathrm{~cm}$ (supplied by Blo Rad Laboratories) containing Dowex 1x4 or Dowex 1x8 is satisfactory.

(2) Wash thoroughly with $0.15 \mathrm{M} \mathrm{HCl}-0.2 \mathrm{M} \mathrm{HF}$ and finally with several colunin volumes of $0.35 \mathrm{M}$ HC1 $-0.8 \mathrm{M}$ HF to ersure complete removal of the aluminum matrix.

(3) Elute most of the retained elements (see Tables A-1 and $\mathrm{A}-2$ ) with $\sim 25 \mathrm{~mL}$ of $4 M \mathrm{HNO}_{3}-1 M \mathrm{HF}$.

(4) Elute tantalum with an additional $225 \mathrm{~mL}$ of $12 \mathrm{M} \mathrm{HNO}_{3}-5 \mathrm{M} \mathrm{HF}$. Collect effluents in separate plastic beakers, evaporate each to dryness and dissolve in 1 ml of HCl-hr solution. Determine elements present by the copper spark method.1,2

\section{b. Cation Exchange}

(1) Pass solution through a sintlar colum containing cationexchange resin. Dowex 50x8, 100-200 mesh, 1s satisfactory. Wash thoroughly with $0.15 \mathrm{M}$ BCl-0.2N HF and finally with a few mL of water.

(2) Elute the retained impurities with 25 ml of $4.5 \mathrm{~N}$ BCl, evaporate to $1 \mathrm{~mL}$, and determine the elements present by the copper spark method.1,2 A number of elements, Including the rare earthe, may be determined by this procedure.9-11

Besults for a previously analyzed sample to which some Impurities were added to test the separation procedures are given In Table 2. 
Table 2. Alumirum Metal Sample (Blution with $0.15 \mathrm{M}$ HCl-0.2M HF; visual comparlson results, $\pm 50 \%$ )

\begin{tabular}{|c|c|c|c|c|}
\hline \multirow[b]{2}{*}{ Impurity } & \multicolumn{2}{|c|}{$\begin{array}{l}\text { Amount Impurity } \\
\text { Present, ppm }\end{array}$} & \multicolumn{2}{|c|}{$\begin{array}{l}\text { Amount Impurity } \\
\text { Found, } \mathrm{ppm}\end{array}$} \\
\hline & Reported & Added & By Anton $\mathrm{X}$ & By Cation X \\
\hline $\mathrm{Ag}$ & $<1$ & 100 & 100 & \\
\hline Co & $<5$ & 50 & & 40 \\
\hline Cd & $<1$ & 100 & & Very Low \\
\hline $\mathrm{Fe}$ & 2000 & - & & Very Low \\
\hline $\mathrm{Ga}$ & 200 & - & & 300 \\
\hline Hf & $<5$ & 100 & 50 & \\
\hline Mn & 40 & - & & 40 \\
\hline $\mathbf{T 1}$ & 60 & -- & 60 & \\
\hline $\boldsymbol{\nabla}$ & 60 & - & & 50 \\
\hline$W$ & $<10$ & 100 & 100 & \\
\hline $\mathbf{L a}$ & $<0.5$ & 50 & & 40 \\
\hline $\mathbf{E u}$ & $<0.5$ & 50 & & 40 \\
\hline Sc & $<0.5$ & 50 & & 0 \\
\hline
\end{tabular}

9. Analysis of Aluminum-Urantum Alloys

A procedure simflar to Procedure 8 has been used for the analysis of aluminum-urantum alloys for trace Impurities. Uranium, 11ke aluminum, is not retained by an anion-exchange resin from dilute HCl-HF nor by a cationexchange resin from dilute $\mathrm{HF}$. Samples are dissolved in $\mathrm{HNO}_{3}-\mathrm{HF}$, evaporated to dryness and taken up in an appropriate solution. The anfon-exchange method is identical to that given for the analysis of aluminum metal. For cationexchange separation, the sample allquot 18 placed in $20.15 \mathrm{M}$ HF and passed through the column. After the colum is washed well with $0.15 \mathrm{M} \mathrm{HF}$, the procedure is carried out in the same way as that described above.

10. Analysis of $80 \%$ Aluminum-20\% Plutonium Alloy

DLs801ve a weighed sample ( $200-300 \mathrm{mg}$ ) in HCl plus some BNO $_{3}$, take to Inciplent dryness and take up in $8 \mathrm{~N} \mathrm{BNO}_{3}$. Pass sample solution through a column containing an anion-exchange resin in the nitrate form to remove the plutonium. 12 Wash thoroughly with $8 \mathrm{BHO}_{3}$. Divide the effluent into three equal portions and evaporate to dryness.

Portion A. Take up In Ellute BCI-IIF and pase through a emall cationaxchange resin colum. Proceed as described above for aluninum analyeis. 
Portion B. Take up in $0.1 \mathrm{M} \mathrm{HC1-0.5M} \mathrm{HF} \mathrm{and} \mathrm{pass} \mathrm{through} \mathrm{a} \mathrm{small}$ preconditioned anion -exchange column. Wash thoroughly with $0.1 M$ HCl-0.5M HF. Elute the adsorbed Impurities with $4 M \mathrm{BNO}_{3}-1 \mathrm{M}$ HF and proceed as described for analysis of high-purity aluminum.

Portion C. Take up in $6 M$ HCl and pass through a small preconditioned anlon-exchange column. Wash thoroughly with $6 \mathrm{M} \mathrm{HCl,} \mathrm{then} \mathrm{elute} \mathrm{the} \mathrm{adsorbed}$ elements ( $\mathrm{Fe}, \mathrm{Ga}, \mathrm{Te}, \mathrm{Cd}, \mathrm{U}, \mathrm{Zn}$ ) with dilute HCl. Evaporate to a small volume and determine impurities present by the copper spark method.1,2

\section{REFERENCES}

1. M. Yred, N. H. Nachtrieb, and F. S. Tomkins, Spectrochemical Analysis by the Copper Spark Method, J. Opt. Soc. An. 37, 279 (1947).

2. J. P. Faris, Application of the Copper Spark Method for Spectrographic Analysis, Proc. 6th Conf. Anal. Chem. Nuc1. Reactor Tech., Gatilnburg, Tenn., 1962, TID-7655, p. 193 (1962).

3. J. P. Faris and J. K. Brody, Adsorption of Elements from HCl-HF Mixtures, Paper No. 34, P1tteburgh Conf. on Anal. Ch'em. and Appl. Spec., 1961.

4. J. P. Faris, Adsorption of the Elements from Hydrufluoric Acid by Anion Exchange, Ana1. Chem. 32, 520 (1960).

5. E. A. Huff, Anton Exchange Study of a Number of Blemente in NitricBydrofluoric Acld Metxtures. Analytical Applications of the System, Anal. Chem. 36, 1921 (1964).

6. J. M. Morris and F. X. P1nk, Symposium on Spectrochemical Analysis for Trace Element8, ASTM Spec. Tech. Pub. No. 221, p. 39, (1957).

7. E. A. Buff, Trace Impur1ty Analye1s of Thorfum-Uranium and PlutontumThortum-Uranium Alloys by AnIon Exchange-Partition Chromatography, Anal. Chem. 37, 533 (1965).

7a. E. A. Huff and S. J. Kulpa, Trace Impurity Analysis of Plutonium-UraniumZirconium AlloyB by Anion Bxchange - Partition Chromatography, Anal. Chem. 38, 939 (1966).

8. B. F. Scribner and B. R. Mullin, Carrier-Dist1llation Mothod for Spectrographic Analysis and Its Application to the Anelyels of Uranium-Base Yateriale, J. Res. Nat1. Bur. Stand. 37, 379 (1946).

9. J. S. Fritr, B.' B. Garralda, and S. R. Karraker, Catton Exchange Soparation of Yatal Ions by Blution whth Bydrofluoric Actd, Ana1. Chew. 33, 882 (1961).

10. L. Danialoson and T. Bletrom, Cation Exchange separation of Ireces of

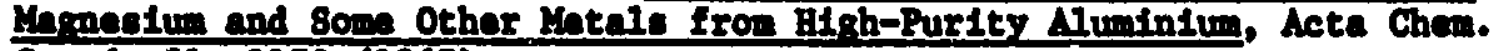
8cand. 21, 1173 (1967). 


\section{REFERENCES (contd)}

11. J. Kork18ch and' A, Huber, Cation-Exchange Behavlour of Several Elements In Hydrofluortc Acid - Organic Solvent Media, Talanta 15, 119 (1968).

12. J. K. Brody, J. P. Faris, and R. F. Buchanan, Ion Exchange Spectrographic Method for Determination of Impurities in Uranium and Plutonium, Anal. Chem. 30, 1909 (1958). 
15

APPBNDIX

DISTRIBUTIO COEFFICIENTS FOR ELEMANTS

IN HCI-HF MIXIURES, HCI, AND HF 
Table A-1. Distribution Coefficiente on Dowex $1 \times 10$ Anion-Bxchange Reain (200-400 mash) as a Punction of HCl and HF Concentrations (concentrations in molarity)

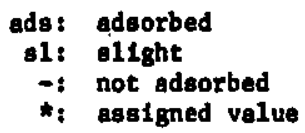

\begin{tabular}{|c|c|c|c|c|c|c|c|c|c|c|c|}
\hline \multirow[b]{2}{*}{ Element } & \multirow[b]{2}{*}{ HF } & \multicolumn{10}{|c|}{ HCl } \\
\hline & & 0 & 0.1 & 0.2 & 0.5 & 1 & 3 & 6 & 9 & 10 & 12 \\
\hline$T 1(I V)$ & $\begin{array}{l}0 \\
0.1 \\
0.2 \\
0.5 \\
1 \\
4 \\
8\end{array}$ & $\begin{array}{r}\text { ads } \\
\text { ads } \\
\text { ads } \\
\text { ads } \\
320 \\
72\end{array}$ & $\begin{array}{r}- \\
\text { ads } \\
\text { ads } \\
\text { ads } \\
\text { ads } \\
290 \\
65\end{array}$ & $\begin{array}{r}- \\
295 \\
390 \\
400 \\
360 \\
158 \\
45\end{array}$ & $\begin{array}{l}- \\
42 \\
49 \\
58 \\
60 \\
44 \\
25\end{array}$ & $\begin{array}{l}15 \\
15 \\
15 \\
15 \\
14 \\
10\end{array}$ & $\begin{array}{l}- \\
4.1 \\
5.4 \\
2.3 \\
1.4 \\
- \\
-\end{array}$ & $\begin{array}{l}- \\
-* \\
- \\
-* \\
- \\
- \\
-\end{array}$ & $\begin{array}{l}- \\
- \\
- \\
- \\
- \\
-\end{array}$ & $\begin{array}{l}24 \\
-* \\
-* \\
-* \\
-* \\
-*\end{array}$ & $\begin{array}{l}55 \\
01 ? \\
- \\
-k \\
-\end{array}$ \\
\hline $\mathrm{zr}(\mathrm{IV})$ & $\begin{array}{l}0 \\
0.1 \\
0.2 \\
0.5 \\
1 \\
4 \\
8\end{array}$ & $\begin{array}{r}\text { ads } \\
\text { ads } \\
\text { ads } \\
\text { ads } \\
320 \\
60\end{array}$ & $\begin{array}{r}- \\
\text { ads } \\
\text { ads } \\
\text { ads } \\
\text { ads } \\
280 \\
58\end{array}$ & $\begin{array}{r}310 \\
400 \\
410 \\
340 \\
140 \\
45\end{array}$ & $\begin{array}{l}-5 \\
39 \\
47 \\
56 \\
58 \\
43 \\
24\end{array}$ & $\begin{array}{l}- \\
7 \\
12 \\
14.5 \\
15 \\
14 \\
7.5\end{array}$ & $\begin{array}{l}- \\
- \\
- \\
- \\
- \\
-\end{array}$ & $\begin{array}{l}- \\
- \\
- \\
- \\
- \\
- \\
-\end{array}$ & $\begin{array}{c}81-30 \\
- \\
- \\
- \\
- \\
= \\
=\end{array}$ & $\begin{array}{r}\text { ade } \\
-* \\
-* \\
-* \\
-* \\
-*\end{array}$ & $\begin{array}{c}\text { ade } \\
- \\
- \\
-k \\
-\end{array}$ \\
\hline HE (IV) & $\begin{array}{l}0 \\
0.1 \\
0.2 \\
0.5 \\
1 \\
4 \\
8\end{array}$ & $\begin{array}{l}\text { ads } \\
\text { ads } \\
\text { ads } \\
\text { ads } \\
325 \\
60\end{array}$ & $\begin{array}{r}\text { ads } \\
\text { ads } \\
\text { ads } \\
\text { ads } \\
280 \\
58\end{array}$ & $\begin{array}{r}- \\
340 \\
400 \\
410 \\
340 \\
140 \\
45\end{array}$ & $\begin{array}{l}-5 \\
52 \\
57 \\
58 \\
43 \\
24\end{array}$ & $\begin{array}{l}- \\
9 \\
13 \\
14.5 \\
15 \\
14 \\
7.5\end{array}$ & $\begin{array}{l}- \\
\overline{-} \\
\overline{-} \\
\overline{-} \\
\bar{z}\end{array}$ & $\begin{array}{l}- \\
- \\
- \\
- \\
- \\
- \\
-\end{array}$ & $\begin{array}{l}4 \\
= \\
- \\
= \\
=\end{array}$ & $\begin{array}{r}\text { (75) } \\
-* \\
-* \\
-\star \\
-* \\
-*\end{array}$ & $\begin{array}{c}\text { ada } \\
- \\
- \\
- \\
-\end{array}$ \\
\hline $\mathrm{Nb}(\mathrm{V})$ & $\begin{array}{l}0 \\
0.1 \\
0.2 \\
0.5 \\
1 \\
4 \\
8\end{array}$ & $\begin{array}{r}\text { ada } \\
\text { ada } \\
\text { ada } \\
\text { ada } \\
2900 \\
180\end{array}$ & $\begin{array}{r}w 75 \\
2500 \\
2625 \\
2720 \\
475 \\
135\end{array}$ & $\begin{array}{l}150 \\
155 \\
180 \\
190 \\
190 \\
108\end{array}$ & $\begin{array}{l}61 \\
59 \\
59 \\
59 \\
59 \\
75\end{array}$ & $\begin{array}{l}35 \\
30 \\
28 \\
28 \\
35 \\
55\end{array}$ & $\begin{array}{l}13.5 \\
13.5 \\
14 \\
14 \\
37 \\
90\end{array}$ & $\begin{array}{c}4.8 \\
6 \\
11 \\
20 \\
97 \\
123\end{array}$ & $\begin{array}{r}14 \\
7 \\
18 \\
40 \\
125 \\
85\end{array}$ & $\begin{array}{l}35 \\
12 \\
23 \\
50 \\
95\end{array}$ & $\begin{array}{r}121 \\
42 \\
34 \\
71\end{array}$ \\
\hline $\operatorname{Ta}(\boldsymbol{V})$ & $\begin{array}{l}0 \\
0.1 \\
0.2 \\
0.5 \\
1 \\
4 \\
8\end{array}$ & $\begin{array}{l}\text { ado } \\
\text { ade } \\
\text { ade } \\
\text { ado } \\
\text { ade } \\
410\end{array}$ & $\begin{array}{l}\text { ade } \\
\text { ade } \\
\text { ade } \\
\text { ade } \\
\text { ade } \\
\text { n90 }\end{array}$ & $\begin{array}{l}\text { ade } \\
\text { ade } \\
\text { ade } \\
\text { ade } \\
\text { ada } \\
\text { w40 }\end{array}$ & $\begin{array}{l}\text { ads } \\
\text { ade } \\
\text { ade } \\
\text { ade } \\
\text { ade } \\
2360\end{array}$ & $\begin{array}{l}\text { ads } \\
\text { ade } \\
\text { ade } \\
\text { ads } \\
\text { ade } \\
\text { v295 }\end{array}$ & $\begin{array}{l}\text { ads } \\
\text { ade } \\
\text { ade } \\
\text { ade } \\
\text { ads } \\
175\end{array}$ & $\begin{array}{l}\text { ade } \\
\text { ads } \\
\text { ade } \\
\text { ads } \\
275 \\
104\end{array}$ & $\begin{array}{r}\text { ed } \\
390 \\
325 \\
275 \\
143 \\
65\end{array}$ & $\begin{array}{c}230 \\
230 * \\
220 * \\
\checkmark 180 \\
90\end{array}$ & $\begin{array}{l}125 \\
122 \\
115 \\
102\end{array}$ \\
\hline H(VI) & $\begin{array}{l}0 \\
0.1 \\
0.2 \\
0.5 \\
1 \\
4 \\
8\end{array}$ & $\begin{array}{l}\text { ade } \\
\text { ade } \\
\text { ade } \\
\text { adi } \\
\text { ade } \\
110\end{array}$ & $\begin{array}{r}v 475 \\
v 750 \\
v 1150 \\
v 1400 \\
v 560 \\
95\end{array}$ & $\begin{array}{r}115 \\
158 \\
208 \\
230 \\
185 \\
75\end{array}$ & $\begin{array}{l}36 \\
45 \\
52 \\
53 \\
52 \\
35\end{array}$ & $\begin{array}{l}17.5 \\
22 \\
24 \\
25 \\
22 \\
14\end{array}$ & $\begin{array}{l}11 \\
13 \\
12.5 \\
11 \\
5.2 \\
11\end{array}$ & $\begin{array}{c}10 \\
8 \\
4.5 \\
1 \\
. . \\
=\end{array}$ & $\begin{array}{r}36 \\
26 \\
12 \\
6 \\
-1 \\
5\end{array}$ & $\begin{array}{l}54 \\
36 \\
20 k \\
12\end{array}$ & $\begin{array}{l}50 \\
42 \\
29 \\
21\end{array}$ \\
\hline $\operatorname{sn}(\mathrm{IV})$ & $\begin{array}{l}0 \\
0.1 \\
0.2 \\
0.3 \\
1 \\
4 \\
8\end{array}$ & $\begin{array}{l}\text { ado } \\
\text { ade } \\
\text { ade } \\
\text { ade } \\
230 \\
64\end{array}$ & $\begin{array}{l}\text { ppt } \\
\text { ade } \\
\text { ado } \\
\text { ado } \\
\text { ede } \\
v 300 \\
57\end{array}$ & $\begin{array}{c}\text { (ppe)? } \\
340 \\
380 \\
390 \\
360 \\
153 \\
44\end{array}$ & $\begin{array}{l}315 \\
58 \\
62.5 \\
66 \\
64 \\
41 \\
24\end{array}$ & $\begin{array}{l}\text { ade } \\
10 \\
18 \\
26 \\
22 \\
15 \\
10.5\end{array}$ & $\begin{array}{l}\text { eds } \\
\text { ade } \\
\text { ads } \\
165 \\
58 \\
15 \\
12\end{array}$ & $\begin{array}{l}\text { ado } \\
\text { ade } \\
\text { ade } \\
\text { ads } \\
\text { ade } \\
\text { w280 } \\
\text { w20s }\end{array}$ & $\begin{array}{r}\text { ade } \\
\text { ade } \\
\text { ade } \\
\text { ado } \\
\text { ads } \\
\text { ads } \\
\text { vioos }\end{array}$ & $\begin{array}{l}\text { ads } \\
\text { adnk } \\
\text { adok } \\
\text { adok } \\
\text { adek } \\
\text { ado }\end{array}$ & $\begin{array}{l}\text { ado } \\
\text { ade } \\
\text { ade } \\
\text { ade }\end{array}$ \\
\hline $\mathrm{H}(\mathrm{VI})$ & $\begin{array}{l}0 \\
0.1 \\
0.2 \\
0.3 \\
1 \\
4 \\
8\end{array}$ & $\begin{array}{l}\text { ade } \\
\text { ade } \\
\text { ade } \\
\text { ade } \\
185 \\
70\end{array}$ & $\begin{array}{c}(1115) \\
1160 \\
w 230 \\
w 300 \\
w 320 \\
273 \\
56\end{array}$ & $\begin{array}{c}\text { (438) } \\
68 \\
99 \\
212 \\
110 \\
86 \\
49\end{array}$ & $\begin{array}{c}\text { (416) } \\
31 \\
34 \\
42 \\
44 \\
34 \\
24\end{array}$ & $\begin{array}{l}(49) \\
24 \\
25 \\
25.5 \\
25 \\
17.9 \\
14\end{array}$ & $\begin{array}{l}98 \\
35 \\
32 \\
23.5 \\
15.5 \\
7 \\
5.5\end{array}$ & $\begin{array}{r}210 \\
170 \\
140 \\
72 \\
40 \\
14 \\
10\end{array}$ & $\begin{array}{c}153 \\
130 \\
130 \\
120 \\
59 \\
(298) \\
2957\end{array}$ & $\begin{array}{r}215 \\
2140 \\
2130 \\
2110 \\
(210)\end{array}$ & $\begin{array}{l}150-250 \\
(w 280) \\
(w 275) \\
(n 265) \\
(n 250)\end{array}$ \\
\hline
\end{tabular}


Tab10 A-1. (coatd)

\begin{tabular}{|c|c|c|c|c|c|c|c|c|c|c|c|}
\hline \multirow[b]{2}{*}{ Element } & \multirow[b]{2}{*}{ HF } & \multicolumn{10}{|c|}{$\mathrm{HC1}$} \\
\hline & & 0 & 0.1 & 0.2 & 0.5 & 1 & 3 & 6 & 9 & 10 & 12 \\
\hline $\operatorname{Re}(V I I)$ & $\begin{array}{l}0 \\
0.1 \\
0.2 \\
0.5 \\
1 \\
4 \\
8\end{array}$ & $\begin{array}{l}\text { ada } \\
\text { ada } \\
\text { ads } \\
\text { ads } \\
\text { ada } \\
\text { its }\end{array}$ & $\begin{array}{l}\text { ade } \\
\text { ade } \\
\text { ads } \\
\text { ads } \\
2770 \\
185\end{array}$ & $\begin{array}{l}\text { ade } \\
\text { ade } \\
\text { ado } \\
\text { ads } \\
\text { ade } \\
\text { 550* } \\
176\end{array}$ & $\begin{array}{l}\text { ade } \\
\text { ads } \\
\text { ads } \\
\text { ado } \\
\text { ads } \\
340 \\
128\end{array}$ & $\begin{array}{r}\text { ade } \\
\text { ada } \\
\text { ade } \\
\text { ado } \\
\text { ade } \\
\text { 215 } \\
85\end{array}$ & $\begin{array}{r}380 \\
\text { ade } \\
\text { ade } \\
280 \\
180 \\
72 \\
32\end{array}$ & $\begin{array}{l}81 \\
75 \\
75 \\
62 \\
47 \\
21 \\
14\end{array}$ & $\begin{array}{l}22.5 \\
21 \\
21 \\
16.3 \\
15 \\
14 \\
(15-80)\end{array}$ & $\begin{array}{l}16.5 \\
16 \\
16 \star \\
16 \star \\
16 \\
16\end{array}$ & $\begin{array}{l}26 \\
23 \\
25 \\
24 \\
27\end{array}$ \\
\hline $\mathrm{H}_{\mathrm{g}}(\mathrm{II})$ & $\begin{array}{l}0 \\
0.1 \\
0.2 \\
0.5 \\
1 \\
4 \\
8\end{array}$ & $\begin{array}{l}81 \\
01 \\
817 \\
= \\
=\end{array}$ & $\begin{array}{l}\text { ads } \\
\text { ads } \\
\text { ads } \\
\text { ads } \\
\text { ads } \\
\text { ads } \\
\text { ads }\end{array}$ & $\begin{array}{l}\text { ade } \\
\text { - ado } \\
\text { ade } \\
\text { ade } \\
\text { ade } \\
\text { ada } \\
\text { ada }\end{array}$ & $\begin{array}{l}\text { ads } \\
\text { ads } \\
\text { ads } \\
\text { ade } \\
\text { ads } \\
\text { adsk } \\
\text { adsk }\end{array}$ & $\begin{array}{l}\text { ada } \\
\text { ads* } \\
\text { ads* } \\
\text { ads } \\
\text { ada } \\
\text { ads } \\
\text { ads }\end{array}$ & $\begin{array}{l}\text { ade } \\
\text { ads* } \\
\text { ada* } \\
\text { ads" } \\
\text { ade* } \\
\text { ada } \\
\text { vilo }\end{array}$ & $\begin{array}{l}\text { ads } \\
\text { ada* } \\
\text { ada* } \\
\text { ada* } \\
\text { ada* } \\
255 \\
2100\end{array}$ & $\begin{array}{r}200 \\
165 \\
164 \\
140 \\
116 \\
69 \\
37\end{array}$ & $\begin{array}{l}w 120 \\
w 110 \\
w 108 * \\
w 92 \star \\
76 \\
45\end{array}$ & $\begin{array}{l}58 \\
58 \\
58 \\
48 \\
45\end{array}$ \\
\hline $\operatorname{Pd}(\mathrm{II})$ & $\begin{array}{l}0 \\
0.1 \\
0.2 \\
0.5 \\
1 \\
4 \\
8\end{array}$ & $\begin{array}{l}\text { ade } \\
\text { ads } \\
\text { ade } \\
\text { ade - }\end{array}$ & $\begin{array}{l}\text { ada } \\
\text { ada } \\
\text { ada } \\
\text { ads } \\
\text { ads } \\
\text { ads } \\
\text { ads }\end{array}$ & $\begin{array}{l}\text { ads } \\
\text { sds } \\
\text { ada } \\
\text { ada } \\
\text { adn* } \\
\text { ads* } \\
\text { ads }\end{array}$ & $\begin{array}{r}\text { ads } \\
\text { ado } \\
\text { ads } \\
\text { ads } \\
\text { ads } \\
\text { ads } \\
\text { v } 400\end{array}$ & $\begin{array}{r}\text { ada } \\
\sim 2300 \\
\sim 2200 \\
\sim 1900 \\
\sim 1500 \\
\sim 850 \\
490\end{array}$ & $\begin{array}{r}3500 \\
5.10 \\
500 \\
450 \\
385 \\
190 \\
95\end{array}$ & $\begin{array}{r}120 \\
119 \\
212 \\
102 \\
91 \\
54 \\
33\end{array}$ & $\begin{array}{l}35 \\
45 \\
45 \\
43 \\
40 \\
27 \\
17\end{array}$ & $\begin{array}{c}38 \\
235 \\
35 \star \\
33 \star \\
30 \\
22\end{array}$ & $\begin{array}{l}28 \\
26 \\
26 \\
25 \\
25\end{array}$ \\
\hline$B 1$ (III) & $\begin{array}{l}0 \\
0.1 \\
0.2 \\
0.5 \\
1 \\
4 \\
8\end{array}$ & $\begin{array}{r}33 \\
14 \\
- \\
= \\
=\end{array}$ & $\begin{array}{c}\text { ppt } \\
\text { ads } \\
\text { ade } \\
2580 \\
106 \\
16 \\
10.5\end{array}$ & $\begin{array}{r}\text { ads } \\
\text { ads } \\
\text { ads } \\
\text { ads } \\
22500 \\
225 \\
145\end{array}$ & $\begin{array}{l}\text { ads } \\
\text { ade } \\
\text { ads } \\
\text { ads } \\
\text { ads } \\
\text { ado } \\
\text { ade }\end{array}$ & $\begin{array}{l}\text { ado } \\
\text { ado } \\
\text { ads } \\
\text { ads } \\
\text { ads } \\
\text { ads } \\
\text { ads }\end{array}$ & $\begin{array}{l}\text { ads } \\
\text { ads } \\
\text { ade } \\
\text { ads } \\
w 1400 \\
\sim 500 \\
\sim 220\end{array}$ & $\begin{array}{r}202 \\
200 \\
198 \\
178 \\
156 \\
85 \\
48\end{array}$ & $\begin{array}{l}78 \\
56 \\
65 \\
64 \\
59 \\
40 \\
28\end{array}$ & $\begin{array}{l}57 \\
50 \\
50 \hbar \\
50 \star \\
50 \\
34\end{array}$ & $\begin{array}{l}43 \\
43 \\
43 \\
41 \\
38\end{array}$ \\
\hline $\mathrm{Pb}$ (II) & $\begin{array}{l}0 \\
0.1 \\
0.2 \\
0.5 \\
1 \\
4 \\
8\end{array}$ & $\begin{array}{l}= \\
= \\
= \\
=\end{array}$ & $\begin{array}{l}- \\
- \\
(4) \\
(4.5) \\
(5) \\
(4)\end{array}$ & $\begin{array}{l}11 \\
(10) \\
(10) \\
(9) \\
(8.5) \\
(8) \\
(5)\end{array}$ & $\begin{array}{l}19 \\
18 \\
17.5 \\
16 \\
15 \\
12 \\
10\end{array}$ & $\begin{array}{l}24 \\
22 \\
22 \\
21 \\
20 \\
15 \\
14\end{array}$ & $\begin{array}{l}15 \\
14 \\
14 \\
14 \\
14 \\
6.5 \\
5.5\end{array}$ & $\begin{array}{l}23 \\
= \\
= \\
= \\
=\end{array}$ & $\begin{array}{l}- \\
-* \\
-* \\
-* \\
- \\
-\end{array}$ & $\begin{array}{l}- \\
-* \\
-* \\
-* \\
-* \\
-\end{array}$ & $\begin{array}{l}- \\
\overline{-} \\
\dot{-}\end{array}$ \\
\hline In(III) & $\begin{array}{l}0 \\
0.1 \\
0.2 \\
0.5 \\
1 \\
4 \\
8\end{array}$ & $\begin{array}{r}50 \\
-23 \\
-1 \\
- \\
= \\
=\end{array}$ & $\begin{array}{l}\overline{-} \\
\bar{z} \\
\bar{z}\end{array}$ & $\begin{array}{l}11 \\
= \\
= \\
= \\
=\end{array}$ & $\begin{array}{l}01 \\
01 \\
01 \\
017 \\
= \\
=\end{array}$ & $\begin{array}{c}10 \\
8 \\
6 \\
6 \\
4.4 \\
1 \\
1\end{array}$ & $\begin{array}{l}20 \\
18 \\
17 \\
16 \\
14.5 \\
13.5 \\
10\end{array}$ & $\begin{array}{l}18 \\
16.5 \\
16 \\
15 \\
13 \\
11 \\
7.5\end{array}$ & $\begin{array}{l}13 \\
12 \\
12 \\
12 \\
10 \\
6.5 \\
5.5\end{array}$ & $\begin{array}{l}11 \\
11 \\
11 * \\
10 * \\
8.5 \\
5\end{array}$ & $\begin{array}{l}7.5 \\
7 \\
7 \\
6.5 \\
6\end{array}$ \\
\hline $\mathbf{v}$ & $\begin{array}{l}0 \\
0.1 \\
0.2 \\
0.5 \\
1 \\
4 \\
8\end{array}$ & $\begin{array}{l}(>400) \\
(>200) \\
(10-250) \\
(20-280) \\
(55) \\
(23)\end{array}$ & $\begin{array}{c}- \\
(6) \\
(14) \\
(35) \\
(60) \\
(42) \\
(18)\end{array}$ & $\begin{array}{c}- \\
- \\
- \\
(10) \\
(22) \\
(21) \\
14\end{array}$ & $\begin{array}{c}- \\
- \\
- \\
\overline{(8)} \\
(11) \\
10\end{array}$ & $\begin{array}{l}= \\
= \\
10\end{array}$ & $\begin{array}{l}- \\
= \\
= \\
=\end{array}$ & $\begin{array}{l}- \\
= \\
= \\
= \\
=\end{array}$ & $\begin{array}{l}- \\
= \\
= \\
= \\
-\end{array}$ & $\begin{array}{l}-* \\
-* \\
-* \\
-* \\
-* \\
-*\end{array}$ & $\begin{array}{l}- \\
- \\
-\end{array}$ \\
\hline U(VI) & $\begin{array}{l}0 \\
0.1 \\
0.2 \\
0.5 \\
1 \\
1 \\
8\end{array}$ & $\begin{array}{c}\text { ade } \\
\text { adek } \\
\text { cdet } \\
690 \\
72 \\
23\end{array}$ & $\begin{array}{c}- \\
-1 \\
8 \\
15.3 \\
21 \\
15\end{array}$ & 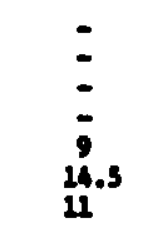 & $\begin{array}{l}- \\
- \\
= \\
01\end{array}$ & $\begin{array}{l}1.2 \\
= \\
= \\
= \\
-\end{array}$ & $\begin{array}{r}35 \\
18 \\
16 \\
6 \\
-1 \\
- \\
=\end{array}$ & $\begin{array}{r}\text { ade } \\
\text { ade } \\
\text { ade } \\
2260 \\
120 \\
33 \\
26\end{array}$ & $\begin{array}{l}\text { ado } \\
\text { ado } \\
\text { ado } \\
\text { ads } \\
\text { aso } \\
350 \\
210\end{array}$ & $\begin{array}{l}\text { ado } \\
\text { adek } \\
\text { edst } \\
\text { adst } \\
\text { adsk }\end{array}$ & $\begin{array}{l}\text { ade } \\
\text { edet } \\
\text { edot } \\
\text { adot } \\
\text { edot }\end{array}$ \\
\hline$T 0(\pi v)$ & $\begin{array}{l}0 \\
0.1 \\
0.2 \\
0.5 \\
1 \\
4 \\
8\end{array}$ & $\begin{array}{l}155 \\
170 \\
172 \\
158 \\
68 \\
36\end{array}$ & $\begin{array}{l}- \\
= \\
12 \\
31 \\
35\end{array}$ & $\begin{array}{c}- \\
= \\
117 \\
14.5 \\
47\end{array}$ & $\begin{array}{c}- \\
- \\
- \\
22 \\
23\end{array}$ & $\begin{array}{l}= \\
= \\
14\end{array}$ & $\begin{array}{r}730 \\
2 d 0 \\
2 d 0 \\
270 \\
230 \\
30 \\
32\end{array}$ & $\begin{array}{l}\text { ado } \\
\text { ad } \\
\text { ad } \\
\text { ade } \\
\text { adr } \\
\text { ade } \\
\text { ade }\end{array}$ & $\begin{array}{l}\text { ad } \\
\text { ads } \\
\text { als } \\
\text { che } \\
\text { ad } \\
\text { ad }\end{array}$ & $\begin{array}{l}\text { ade } \\
\text { adat } \\
\text { adat } \\
\text { adek }\end{array}$ & $\begin{array}{l}\text { ads } \\
\text { ads } \\
\text { ade } \\
\text { adnt }\end{array}$ \\
\hline
\end{tabular}


Table A-1. (contd)

\begin{tabular}{|c|c|c|c|c|c|c|c|c|c|c|c|}
\hline \multirow[b]{2}{*}{ Blement } & \multirow[b]{2}{*}{ HF } & \multicolumn{10}{|c|}{$\mathrm{HCl}$} \\
\hline & & 0 & 0.1 & 0.2 & 0.5 & 1 & 3 & 6 & 9 & 10 & 12 \\
\hline Fe(III) & $\begin{array}{l}0 \\
0.1 \\
0.2 \\
0.5 \\
1 \\
4 \\
8\end{array}$ & $\begin{array}{r}200 \\
80 \\
24 \\
10 \\
- \\
-\end{array}$ & $\begin{array}{l}= \\
= \\
=\end{array}$ & $\begin{array}{l}- \\
- \\
- \\
-\end{array}$ & $\begin{array}{l}- \\
- \\
- \\
- \\
-\end{array}$ & $\begin{array}{c}21.5 \\
= \\
= \\
=\end{array}$ & $\begin{array}{c}(45) \\
(250) \\
(235) \\
12 \\
4 \\
= \\
=\end{array}$ & $\begin{array}{l}\text { ads } \\
\text { ade } \\
\text { ade } \\
\text { ade* } \\
\text { ado* } \\
\text { ads } \\
\text { ads }\end{array}$ & $\begin{array}{l}\text { ads } \\
\text { ads" } \\
\text { ads } \\
\text { ads } \\
\text { ads } \\
\text { ads } \\
\text { ads }\end{array}$ & $\begin{array}{l}\text { ads } \\
\text { ade* } \\
\text { ads* } \\
\text { ads* } \\
\text { ads* } \\
\text { ads* }\end{array}$ & $\begin{array}{l}\text { ads } \\
\text { ade } \\
\text { adot } \\
\text { adet }\end{array}$ \\
\hline $\operatorname{co}(I I)$ & $\begin{array}{l}0 \\
0.1 \\
0.2 \\
0.5 \\
1 \\
4 \\
0\end{array}$ & $\begin{array}{l}= \\
= \\
= \\
=\end{array}$ & $\begin{array}{l}= \\
= \\
= \\
= \\
=\end{array}$ & $\begin{array}{l}- \\
- \\
- \\
- \\
-\end{array}$ & $\begin{array}{l}- \\
\overline{-} \\
\overline{-} \\
\overline{-}\end{array}$ & $\begin{array}{l}- \\
- \\
- \\
- \\
-\end{array}$ & $\begin{array}{l}- \\
\overline{-} \\
\overline{-} \\
\overline{-} \\
\overline{-}\end{array}$ & $\begin{array}{r}9 \\
8 \\
8 \\
9 \\
12 \\
17 \\
41\end{array}$ & $\begin{array}{l}71 \\
63 \\
63 \\
61 \\
60 \\
46 \\
33\end{array}$ & $\begin{array}{l}64 \\
59 \\
59 k \\
56 k \\
53 \\
36\end{array}$ & $\begin{array}{l}45 \\
45 \\
44 \\
42 \\
37\end{array}$ \\
\hline Ga(III) & $\begin{array}{l}0 \\
0.1 \\
0.2 \\
0.5 \\
1 \\
4 \\
8\end{array}$ & $\begin{array}{r}45 \\
20 \\
= \\
= \\
= \\
=\end{array}$ & $\begin{array}{l}- \\
= \\
= \\
=\end{array}$ & $\begin{array}{l}= \\
= \\
= \\
=\end{array}$ & $\begin{array}{l}- \\
= \\
= \\
=\end{array}$ & $\begin{array}{c}22 \\
= \\
= \\
=\end{array}$ & $\begin{array}{c}(81-90) \\
(81-60) \\
(=1-50) \\
(81-35) \\
(81-15) \\
=1 \\
81\end{array}$ & $\begin{array}{l}\text { ads } \\
\text { ads } \\
\text { ads } \\
\text { ads } \\
\text { ads } \\
\text { ads } \\
\text { ads }\end{array}$ & $\begin{array}{l}\text { ade } \\
\text { ade } \\
\text { ads } \\
\text { ade } \\
\text { ads } \\
\text { ade } \\
\text { ade }\end{array}$ & $\begin{array}{l}\text { ade } \\
\text { ade* } \\
\text { ade* } \\
\text { adat } \\
\text { ads" } \\
\text { ads }\end{array}$ & $\begin{array}{l}\text { ada } \\
\text { ads } \\
\text { ads } \\
\text { adsk } \\
\text { ads }\end{array}$ \\
\hline $\cos (I I)$ & $\begin{array}{l}0 \\
0.1 \\
0.2 \\
0.5 \\
1 \\
4 \\
8\end{array}$ & $\begin{array}{l}- \\
- \\
- \\
= \\
=\end{array}$ & $\begin{array}{r}400 \\
2380 \\
2355 \\
2300 \\
2225 \\
250 \\
25\end{array}$ & $\begin{array}{r}\text { ade } \\
\text { ade } \\
2560 \\
w 550 \\
w 325 \\
180 \\
105\end{array}$ & $\begin{array}{l}\text { ads } \\
\text { ads } \\
\text { ads } \\
\text { ads } \\
\text { ads } \\
\text { ads } \\
\text { ada }\end{array}$ & $\begin{array}{l}\text { ads } \\
\text { ads } \\
\text { ada } \\
\text { ads } \\
\text { ads } \\
\text { ads } \\
\text { ads }\end{array}$ & $\begin{array}{l}\text { ade } \\
\text { ads } \\
\text { ads } \\
\text { ads } \\
\text { vs50 } \\
\text { ads } \\
\text { v210 }\end{array}$ & $\begin{array}{r}\checkmark 370 \\
w 315 \\
310 \\
287 \\
220 \\
92 \\
44\end{array}$ & $\begin{array}{l}68 \\
60 \\
59 \\
54 \\
48 \\
26 \\
15\end{array}$ & $\begin{array}{l}36 \\
34 \\
33 * \\
29 * \\
25 \\
15\end{array}$ & $\begin{array}{l}15 \\
15 \\
15 \\
15 \\
15\end{array}$ \\
\hline $\mathrm{Zn}(\mathrm{II})$ & $\begin{array}{l}0 \\
0.1 \\
0.2 \\
0.5 \\
1 \\
4 \\
8\end{array}$ & $\begin{array}{l}- \\
\dot{-} \\
- \\
- \\
-\end{array}$ & $\begin{array}{l}9 \\
25 \\
25 \\
25 \\
25 \\
= \\
=\end{array}$ & $\begin{array}{l}25 \\
21 \\
21 \\
17 \\
16 \\
15 \\
16\end{array}$ & $\begin{array}{r}92 \\
92 \\
92 \\
92 \\
92 \\
88 \\
158\end{array}$ & $\begin{array}{l}\text { ads } \\
\text { ads } \\
\text { ads } \\
\text { ads } \\
\text { ads } \\
\text { ads } \\
290\end{array}$ & $\begin{array}{l}\text { ada } \\
\text { ada } \\
\text { ads } \\
\text { ada } \\
\text { ads } \\
n 300 \\
165\end{array}$ & $\begin{array}{r}245 \\
240 \\
240 \\
210 \\
180 \\
100 \\
57\end{array}$ & $\begin{array}{l}90 \\
84 \\
82 \\
80 \\
75 \\
51 \\
30\end{array}$ & $\begin{array}{l}68 \cdots \\
65 \\
63 \star \\
59 * \\
52 \\
35\end{array}$ & $\begin{array}{l}45 \\
45 \\
43 \\
39 \\
36\end{array}$ \\
\hline$A s(V)$ & $\begin{array}{l}0 \\
0.1 \\
0.2 \\
0.5 \\
1 \\
4 \\
0\end{array}$ & $\begin{array}{c}160 \\
59 \\
(40-140) \\
(170-260) \\
(230) \\
95\end{array}$ & $\begin{array}{c}= \\
= \\
= \\
1 \\
230 \\
(190-240) \\
92\end{array}$ & $\begin{array}{r}= \\
\overline{-} \\
\overline{6} \\
2170 \\
88\end{array}$ & $\begin{array}{r}= \\
= \\
= \\
6100 \\
65\end{array}$ & $\begin{array}{l}- \\
= \\
= \\
= \\
11 \\
65 \\
46\end{array}$ & $\begin{array}{c}- \\
- \\
-\ldots \\
- \\
(5-15) \\
28 \\
22\end{array}$ & 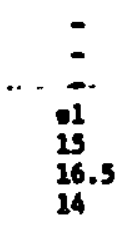 & $\begin{array}{l}- \\
- \\
- \\
14 \\
12 \\
10\end{array}$ & $\begin{array}{l}- \\
- \\
14 \\
11\end{array}$ & $\begin{array}{c}-18 \\
\overline{7} \\
14\end{array}$ \\
\hline B(III) & $\begin{array}{l}0 \\
0.1 \\
0.2 \\
0.5 \\
1 \\
4 \\
8\end{array}$ & $\begin{array}{l}\text { ads } \\
\text { ads } \\
\text { ads } \\
\text { ads } \\
200 \\
56\end{array}$ & $\begin{array}{l}\text { ads } \\
\text { ado } \\
\text { ads } \\
2900 \\
2190 \\
69\end{array}$ & $\begin{array}{r}w 570 \\
w 540 \\
w 510 \\
410 \\
142 \\
53\end{array}$ & $\begin{array}{r}280 \\
250 \\
220 \\
188 \\
75 \\
38\end{array}$ & $\begin{array}{l}143 \\
130 \\
112 \\
94 \\
48 \\
26\end{array}$ & $\begin{array}{l}45 \\
43 \\
38 \\
32 \\
19 \\
12\end{array}$ & $\begin{array}{l}19 \\
17 \\
15 \\
14.5 \\
10 \\
7.5\end{array}$ & $\begin{array}{c}-5 \\
10 \\
7.5 \\
5.5 \\
5 \\
7 \\
6.5\end{array}$ & $\begin{array}{l}\overline{9} \\
9 k \\
7 k \\
25 \\
26\end{array}$ & $5^{5}$ \\
\hline 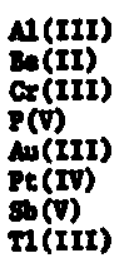 & $\begin{array}{l}0.1-8 \\
0.1-8 \\
0.1-8 \\
0.1-8 \\
0.1-8 \\
0.1-8 \\
0.1-8 \\
0.1-8\end{array}$ & & $\begin{array}{l}\dot{-} \\
\dot{-} \\
\text { ads } \\
\text { ads }\end{array}$ & $\begin{array}{r}\ddot{E} \\
\ddot{E} \\
\text { ade } \\
\text { ade }\end{array}$ & $\begin{array}{l}\overline{-} \\
\overline{-} \\
\text { eds } \\
\text { ade }\end{array}$ & $\begin{array}{l}- \\
\text { ads } \\
\text { ads }\end{array}$ & i & $\begin{array}{l}\overline{-} \\
\overline{-} \\
\text { ade } \\
\text { ado } \\
\text { ado }\end{array}$ & $\begin{array}{l}\overline{-} \\
\dot{-} \\
\text { ads } \\
\text { ade } \\
\text { ade }\end{array}$ & $\begin{array}{l}\text { - } \\
\text { ado } \\
\text { ads } \\
\text { ade }\end{array}$ & $\begin{array}{l}\overline{-} \\
\bar{a} \\
\text { ads } \\
\text { adi }\end{array}$ \\
\hline
\end{tabular}




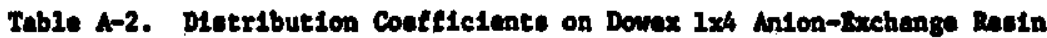
(100-200 ansh) a Dunct10n of HCL and tis Concentrations (concentrations in molarity)

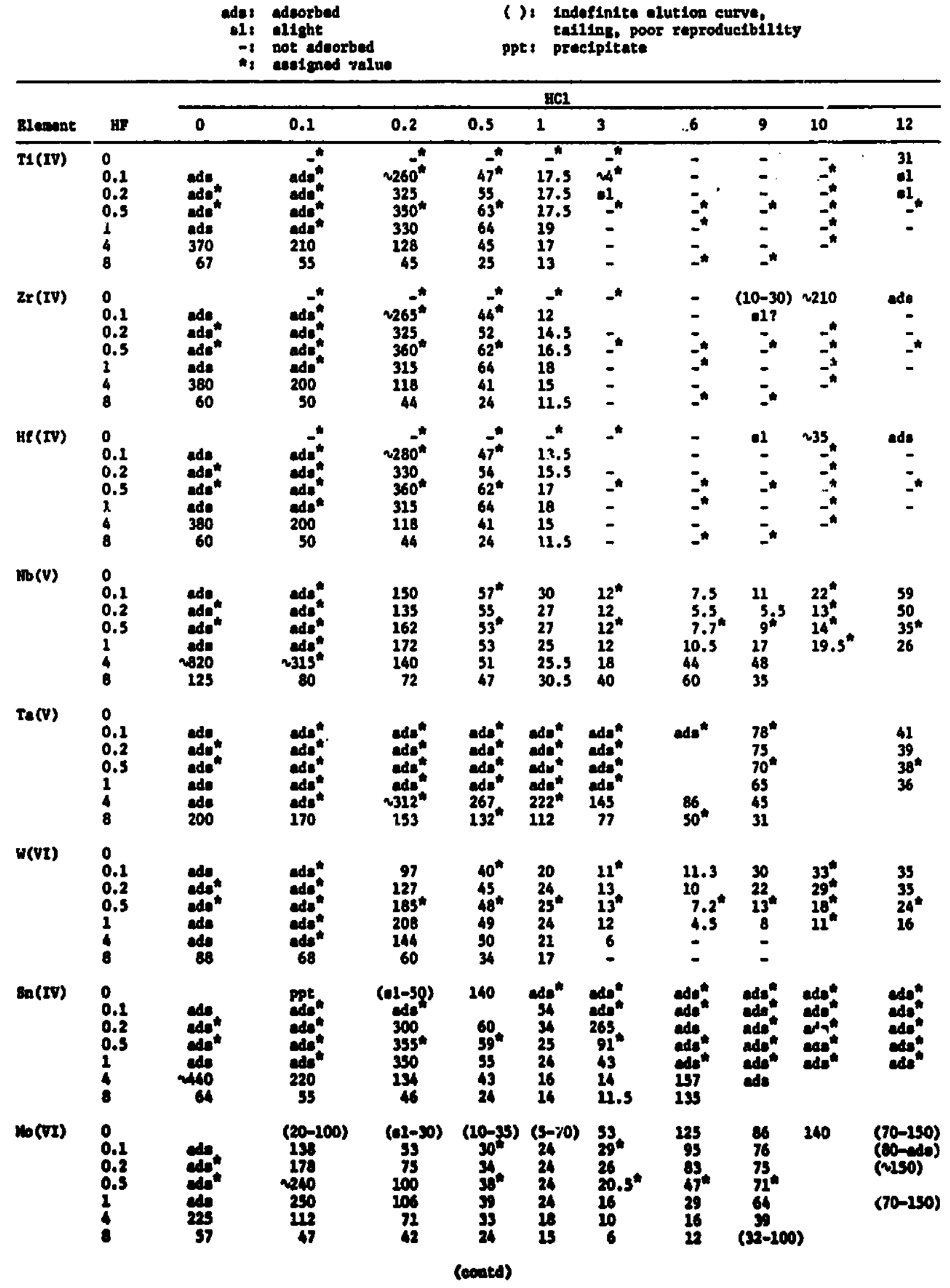


Table A-2. (contd)

\begin{tabular}{|c|c|c|c|c|c|c|c|c|c|c|c|}
\hline \multirow[b]{2}{*}{ Blemont } & \multirow[b]{2}{*}{ HF } & \multicolumn{10}{|c|}{$\mathrm{ACl}$} \\
\hline & & 0 & 0.1 & 0.2 & 0.5 & 1. & 3 & 6 & 9 & 10 & 12 \\
\hline$R \bullet(V I I)$ & $\begin{array}{l}0 \\
0.1 \\
0.2 \\
0.5 \\
1 \\
4 \\
8\end{array}$ & $\begin{array}{l}\text { ads } \\
\text { ads } \\
\text { ads" } \\
\text { ads } \\
\text { ads } \\
112\end{array}$ & $\begin{array}{l}\text { ads" } \\
\text { ads" } \\
\text { ads" } \\
\text { ads" } \\
340 \\
105\end{array}$ & $\begin{array}{l}\text { ads" } \\
\text { ada" } \\
\text { ads" } \\
\text { ads" } \\
\text { ads" } \\
2 B 2 \\
\text { v105 }\end{array}$ & $\begin{array}{l}\text { ads" } \\
\text { ads" } \\
\text { ads" } \\
\text { ads" } \\
\text { ads" } \\
170 \\
82 *\end{array}$ & $\begin{array}{l}\text { ada" } \\
\text { ads" } \\
\text { ads" } \\
\text { ada" } \\
\text { ads" } \\
100 \\
54\end{array}$ & $\begin{array}{c}107 \\
107 \\
95^{\star} \\
77 \\
35 \\
22\end{array}$ & $\begin{array}{l}29 \\
26 \\
26 \\
23^{\star} \\
20 \\
14 \\
10\end{array}$ & $\begin{array}{l}12 \\
13 \\
13 \\
13^{*} \\
13 \\
13\end{array}$ & 14 & $\begin{array}{l}21 \\
21 \\
24 \\
23^{*} \\
23\end{array}$ \\
\hline Hg(II) & $\begin{array}{l}0 \\
0.1 \\
0.2 \\
0.5 \\
1 \\
4 \\
8\end{array}$ & $\begin{array}{l}81+ \\
81+ \\
- \\
= \\
-\end{array}$ & $\begin{array}{l}\text { ads } \\
\text { ads } \\
\text { ads* } \\
\text { ads* } \\
\text { ads }\end{array}$ & $\begin{array}{l}\text { ads"* } \\
\text { ads" } \\
\text { ads" } \\
\text { ade" } \\
\text { ads } \\
\text { ads }\end{array}$ & $\begin{array}{l}\text { ads" } \\
\text { ads* } \\
\text { ads" } \\
\text { ads" } \\
\text { ads* } \\
\text { ads* } \\
\text { ads* }\end{array}$ & $\begin{array}{l}\text { ads"* } \\
\text { ads*" } \\
\text { ads" } \\
\text { ads* } \\
\text { ads* } \\
\text { ads* } \\
\text { ads* }\end{array}$ & $\begin{array}{l}\text { ods* } \\
\text { ads* } \\
\text { ads* } \\
\text { ads* } \\
\text { ads* } \\
\text { ads" } \\
250\end{array}$ & $\begin{array}{l}2295 \\
2300 \\
2280^{\star} \\
2260^{\star} \\
120 \\
267^{\star}\end{array}$ & $\begin{array}{l}75 \\
77 \\
76 \\
74^{\mathrm{W}} \\
70 \\
41 \\
31\end{array}$ & $\begin{array}{l}54 \\
55^{\star} \\
54^{*} \\
54^{*} \\
53^{*}\end{array}$ & $\begin{array}{l}30 \\
30 \\
30 \\
30^{n} \\
30\end{array}$ \\
\hline $\operatorname{Pd}(I I)$ & $\begin{array}{l}0 \\
0.1 \\
0.2 \\
0.5 \\
1 \\
4 \\
8\end{array}$ & $\begin{array}{l}\text { ads" } \\
\text { ads" } \\
\text { ads* } \\
\text { ads" }\end{array}$ & $\begin{array}{l}\text { ads" } \\
\text { ads } \\
\text { ads } \\
\text { ads* } \\
\text { ads* } \\
\text { ads* } \\
\text { ads" }\end{array}$ & $\begin{array}{l}\text { ads* } \\
\text { ads* } \\
\text { ads" } \\
\text { ads* } \\
\text { ads" } \\
\text { ads* } \\
\text { ade* }\end{array}$ & $\begin{array}{l}\text { ads" } \\
\text { ads" } \\
\text { ads* } \\
\text { ads" } \\
\text { ads" } \\
\text { ads" } \\
\text { ads" }\end{array}$ & $\begin{array}{l}\text { ads* } \\
\text { ads* } \\
\text { ads* } \\
\text { ads* } \\
\text { ads* } \\
\text { ads* } \\
\text { ads* }\end{array}$ & $\begin{array}{l}225 \\
225 \\
212^{\star} \\
190 \\
106 \\
71\end{array}$ & $\begin{array}{l}71 \\
70 \\
68 \\
65^{\star} \\
62 \\
43 \\
30\end{array}$ & $\begin{array}{l}35 \\
35 \\
35 \\
33^{k} \\
31 \\
24 \\
18\end{array}$ & $\begin{array}{l}30 \\
30^{*} \\
30^{\star} \\
29^{\star} \\
28^{\star}\end{array}$ & $\begin{array}{l}24 \\
24 \\
24 \\
23^{\star} \\
21\end{array}$ \\
\hline BI (III) & $\begin{array}{l}0 \\
0.1 \\
0.2 \\
0.5 \\
1 \\
4 \\
8\end{array}$ & $\begin{array}{r}42 \\
24 \\
12 \\
- \\
- \\
-\end{array}$ & $\begin{array}{c}\text { Ppt } \\
\text { ady* } \\
\text { ads* } \\
2220 \\
62 \\
12 \\
5.4\end{array}$ & $\begin{array}{l}\text { ade* } \\
\text { ads } \\
\text { ads" } \\
\text { ads" } \\
\text { ade" } \\
98 \\
71\end{array}$ & $\begin{array}{l}\text { ads* } \\
\text { ads* } \\
\text { ads* } \\
\text { ads" } \\
\text { ads* } \\
\text { ads }\end{array}$ & $\begin{array}{l}\text { ads" } \\
\text { ads* } \\
\text { ads" } \\
\text { ads" } \\
\text { ads" } \\
\text { ads" } \\
\text { ads" }\end{array}$ & $\begin{array}{c}550 \\
\text { ads" } \\
\text { ads* } \\
\text { ads* } \\
\text { ads* } \\
2270 \\
148\end{array}$ & $\begin{array}{l}125 \\
110 \\
110 \\
108^{\star} \\
105 \\
68 \\
45\end{array}$ & $\begin{array}{l}55 \\
54 \\
54 \\
51^{*} \\
49 \\
36 \\
36\end{array}$ & $\begin{array}{l}46 \\
45^{\star} \\
45^{\star} \\
44^{\star} \\
42^{\star}\end{array}$ & $\begin{array}{l}39 \\
38 \\
38 \\
37^{\star} \\
35\end{array}$ \\
\hline $\mathrm{Pb}$ (II) & $\begin{array}{l}0 \\
0.1 \\
0.2 \\
0.5 \\
1 \\
4 \\
8\end{array}$ & $\begin{array}{l}- \\
- \\
- \\
-* \\
-*\end{array}$ & $\begin{array}{l}- \\
- \\
- \\
81 ? \\
81 ? \\
81 ?\end{array}$ & $\begin{array}{c}5.5 \\
(5) ? \\
5.5 \\
7 \\
4 \\
e 1 ? \\
(7) ?\end{array}$ & $\begin{array}{l}14 \\
14^{*} \\
14 \\
14^{\star} \\
14 \\
14 \\
13\end{array}$ & $\begin{array}{l}17.5 \\
16 \\
16 \\
16 \\
15 \\
15 \\
14\end{array}$ & $\begin{array}{l}13 \\
12^{\star} \\
11.5 \\
11{ }^{4} \\
10.5 \\
6.5 \\
17\end{array}$ & $\begin{array}{l}- \\
- \\
-* \\
-\end{array}$ & $\begin{array}{l}= \\
= \\
= \\
-\end{array}$ & & $\begin{array}{l}- \\
= \\
=* \\
-*\end{array}$ \\
\hline In (III) & $\begin{array}{l}0 \\
0.1 \\
0.2 \\
0.5 \\
1 \\
\ddot{8}\end{array}$ & $\begin{array}{r}60 \\
31 \\
13 \\
= \\
= \\
=\end{array}$ & $\begin{array}{l}- \\
= \\
= \\
= \\
-\end{array}$ & $\begin{array}{l}- \\
- \\
z \\
- \\
=\end{array}$ & $\begin{array}{l}6 \\
5.5^{\star} \\
5 \\
- \\
- \\
-\end{array}$ & $\begin{array}{r}11.5 \\
11.5 \\
11.5 \\
10.5 \\
7.5 \\
6.5 \\
7.5\end{array}$ & $\begin{array}{l}18 \\
17.5^{*} \\
17 \\
16.5^{*} \\
15.5 \\
14 \\
12\end{array}$ & $\begin{array}{l}16 \\
16 \\
15,5 \\
15^{\star} \\
14 \\
12 \\
7.5\end{array}$ & $\begin{array}{l}12 \\
12 \\
12 \\
11.5^{\star} \\
11 \\
7.5 \\
5.5\end{array}$ & $\begin{array}{l}10.5 \\
10.5^{*} \\
10.5^{\star} \\
10^{*}\end{array}$ & $\begin{array}{l}8 \\
8 \\
8 \\
8^{*} \\
8\end{array}$ \\
\hline v & $\begin{array}{l}0 \\
0.1 \\
0.2 \\
0.5 \\
1 \\
4 \\
8\end{array}$ & $\begin{array}{l}(>220) \\
(>250) \\
(45-230) \\
(40-300) \\
(62) \\
(24)\end{array}$ & $\begin{array}{c}\overline{7} \\
24 \\
(38) \\
(60) \\
(40) \\
(22)\end{array}$ & $\begin{array}{c}- \\
- \\
11 \\
15 \\
(30) \\
(34) \\
16\end{array}$ & $\begin{array}{l}- \\
= \\
- \\
12 \\
15 \\
13.5\end{array}$ & $\begin{array}{r}- \\
- \\
- \\
\overline{13} \\
9\end{array}$ & $\begin{array}{l}- \\
= \\
= \\
= \\
=\end{array}$ & $\begin{array}{l}- \\
- \\
- \\
-* \\
-\end{array}$ & $\begin{array}{l}- \\
- \\
- \\
-\end{array}$ & & $\begin{array}{l}\overline{-} \\
\overline{-} \\
\overline{-}\end{array}$ \\
\hline$U(V I)$ & $\begin{array}{l}0 \\
0.1 \\
0.2 \\
0.5 \\
1 \\
4 \\
8\end{array}$ & $\begin{array}{l}\text { ada } \\
\text { ade } \\
\text { add" } \\
n 57: \\
61 \\
20\end{array}$ & $\begin{array}{l}- \\
= \\
13.5 \\
25 \\
21 \\
14\end{array}$ & $\begin{array}{l}= \\
- \\
= \\
13 \\
12\end{array}$ & $\begin{array}{l}= \\
= \\
- \\
= \\
-\end{array}$ & $\begin{array}{l}= \\
= \\
= \\
=\end{array}$ & $\begin{array}{r}13 \\
7 \\
= \\
=\end{array}$ & $\begin{array}{c}142 \\
2125 \\
2121 \\
85 \\
52 \\
18 \\
16\end{array}$ & $\begin{array}{l}\text { ads } \\
\text { ads" } \\
\text { ads" } \\
\text { ade* } \\
225 \\
129 \\
90\end{array}$ & $\begin{array}{l}\text { adn } \\
\text { ads" } \\
\text { ads" } \\
\text { ads* } \\
\text { ads" }\end{array}$ & $\begin{array}{l}\text { ado } \\
\text { ada" } \\
\text { adse" } \\
\text { ade" } \\
\text { ade" }\end{array}$ \\
\hline$T e(t V)$ & $\begin{array}{l}0 \\
0.1 \\
0.2 \\
0.5 \\
1 \\
1 \\
8\end{array}$ & $\begin{array}{r}93 \\
107 \\
115 \\
108 \\
56 \\
29\end{array}$ & $\begin{array}{l}= \\
- \\
10 \\
25 \\
43 \\
27\end{array}$ & $\begin{array}{l}= \\
= \\
16 \\
32 \\
26\end{array}$ & $\begin{array}{c}- \\
- \\
- \\
18 \\
16 \\
\text { (eostd) }\end{array}$ & $\begin{array}{l}- \\
= \\
- \\
12\end{array}$ & $\begin{array}{c}123 \\
93 \\
58 \\
35 \\
16 \\
14\end{array}$ & $\begin{array}{l}\text { ads } \\
\text { ads } \\
\text { ads } \\
\text { ads. } \\
\text { ado" } \\
\text { ads" }\end{array}$ & 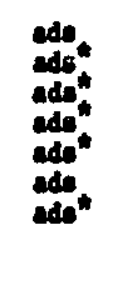 & $\begin{array}{l}\text { ads" } \\
\text { ads" } \\
\text { ado* } \\
\text { ado* } \\
\text { ado* } \\
\text { ada* }\end{array}$ & $\begin{array}{l}\text { ade } \\
\text { ads" } \\
\text { dede" } \\
\text { edo" } \\
\text { ede" }\end{array}$ \\
\hline
\end{tabular}


Table A-2, (contd)

\begin{tabular}{|c|c|c|c|c|c|c|c|c|c|c|c|}
\hline \multirow[b]{2}{*}{ Element } & \multirow[b]{2}{*}{ HE } & \multicolumn{10}{|c|}{ IC1 } \\
\hline & & 0 & 0.1 & 0.2 & 0.5 & 1 & 3 & 6 & 9 & 10 & 12 \\
\hline $\mathrm{P} \in$ (III) & $\begin{array}{l}0 \\
0.1 \\
0.2 \\
0.5 \\
1 \\
4 \\
8\end{array}$ & $\begin{array}{r}265 \\
123 \\
44 \\
20 \\
- \\
-\end{array}$ & $\begin{array}{l}= \\
- \\
- \\
-\star \\
- \\
-\end{array}$ & $\begin{array}{l}= \\
=" \\
= \\
= \\
=\end{array}$ & $\begin{array}{l}-* \\
-* \\
-* \\
-* \\
-*\end{array}$ & $\begin{array}{l}-* \\
- \\
-* \\
= \\
=* \\
-*\end{array}$ & $\begin{array}{l}8 \\
5 \\
= \\
-\end{array}$ & $\begin{array}{l}\text { ads } \\
\text { ads } \\
2295 \\
v 290^{*} \\
v 280^{*} \\
v 230\end{array}$ & $\begin{array}{l}\text { ads" } \\
\text { ads" } \\
\text { ads" } \\
\text { ads" } \\
\text { ads" } \\
\text { ads }\end{array}$ & $\begin{array}{l}\text { ads" } \\
\text { ads" } \\
\text { ads" } \\
\text { ads" } \\
\text { ads" }\end{array}$ & $\begin{array}{l}\text { ads" } \\
\text { adt" } \\
\text { ade* } \\
\text { ade" } \\
\text { eds" }\end{array}$ \\
\hline $\operatorname{co}(I I)$ & $\begin{array}{l}0 \\
0.1 \\
0.2 \\
0.5 \\
1 \\
4 \\
8\end{array}$ & $\begin{array}{l}= \\
- \\
-* \\
-*\end{array}$ & $\begin{array}{l}- \\
=* \\
= \\
= \\
=\end{array}$ & $\begin{array}{l}- \\
\overline{-}^{*} \\
\overline{-} \\
\overline{-}\end{array}$ & $\begin{array}{l}- \\
= \\
-* \\
-* \\
-*\end{array}$ & $\begin{array}{l}- \\
- \\
- \\
- \\
-\end{array}$ & $\begin{array}{l}- \\
- \\
- \\
- \\
-\end{array}$ & $\begin{array}{l}4.5 \\
4.5 \\
4.5 \\
4.5^{\star} \\
4.5 \\
15.5 \\
28\end{array}$ & $\begin{array}{l}40 \\
40 \\
40 \\
38^{*} \\
36 \\
31 \\
26\end{array}$ & $\begin{array}{l}41 \\
41^{*} \\
41^{*} \\
40^{*}\end{array}$ & $\begin{array}{l}30 \\
30 \\
30 \\
29^{\star} \\
28\end{array}$ \\
\hline $\operatorname{Ca}$ (III) & $\begin{array}{l}0 \\
0.1 \\
0.2 \\
0.5 \\
1 \\
4 \\
8\end{array}$ & $\begin{array}{l}68 \\
37 \\
14.5 \\
5.5 \\
- \\
-\end{array}$ & $\begin{array}{l}- \\
- \\
- \\
- \\
- \\
-\end{array}$ & $\begin{array}{l}- \\
- \\
- \\
- \\
- \\
-\end{array}$ & $\begin{array}{l}- \\
-* \\
-^{*} \\
\overline{-}^{*} \\
-^{*}\end{array}$ & $\begin{array}{l}- \\
- \\
- \\
- \\
-\end{array}$ & $\begin{array}{c}(9-30) \\
(5-10) \\
81^{2} \\
= \\
- \\
-\end{array}$ & $\begin{array}{l}\text { ads } \\
\text { ads* } \\
\text { ads } \\
\text { ads" } \\
\text { ads" } \\
\text { ads } \\
\text { ads* }\end{array}$ & $\begin{array}{l}\text { ads } \\
\text { ads" } \\
\text { ads" } \\
\text { ads" } \\
\text { ade* } \\
\text { ade } \\
\text { ads" }\end{array}$ & $\begin{array}{l}\text { ads"* } \\
\text { ads"* } \\
\text { ado" } \\
\text { ads" } \\
\text { ads" }\end{array}$ & $\begin{array}{l}\text { ads } \\
\text { ads" } \\
\text { ads" } \\
\text { adse" } \\
\text { ads" }\end{array}$ \\
\hline Cd(II) & $\begin{array}{l}0 \\
0.1 \\
0.2 \\
0.5 \\
1 \\
4 \\
8\end{array}$ & $\begin{array}{l}- \\
\overline{-} \\
\overline{-} \\
\overline{-}\end{array}$ & $\begin{array}{r}143 \\
120 \\
108 \\
94 \\
71 \\
21 \\
14\end{array}$ & $\begin{array}{r}260 \\
4190 \\
185 \\
160 \\
130 \\
72 \\
60\end{array}$ & $\begin{array}{l}470 \\
\text { ads" } \\
\text { ads } \\
\text { ads" } \\
\text { ads } \\
230 \\
210\end{array}$ & $\begin{array}{l}\text { ads } \\
\text { ads" } \\
\text { ads" } \\
\text { ads } \\
\text { ads } \\
\text { خ } 320 \\
280\end{array}$ & $\begin{array}{c}465 \\
450 \\
425 \star \\
2400 \\
195 \\
105\end{array}$ & $\begin{array}{c}122 \\
117 \\
112 \\
108^{\star} \\
105 \\
58 \\
34\end{array}$ & $\begin{array}{l}31 \\
31 \\
31 \\
30^{\star} \\
28 \\
19 \\
13\end{array}$ & $\begin{array}{l}21 \\
21 * \\
21 * \\
20^{*}\end{array}$ & $\begin{array}{l}12 \\
12 \\
12 \\
12 \\
12\end{array}$ \\
\hline $\mathrm{Zn}(\mathrm{I} T)$ & $\begin{array}{l}0 \\
0.1 \\
0.2 \\
0.5 \\
1 \\
4 \\
8\end{array}$ & $\begin{array}{l}- \\
= \\
= \\
-\end{array}$ & $\begin{array}{l}4.2 \\
=17 \\
= \\
= \\
=\end{array}$ & $\begin{array}{r}11 \\
9 \\
9 \\
9 \\
9 \\
6 \\
13\end{array}$ & $\begin{array}{l}47 \\
46^{*} \\
46 \\
45^{*} \\
40 \\
57 \\
98\end{array}$ & $\begin{array}{l}175^{\star} \\
172^{\star} \\
170 \\
162^{\star} \\
151 \\
135 \\
205\end{array}$ & $\begin{array}{c}320 \\
310 \\
308 \\
303^{*} \\
288 \\
151 \\
99\end{array}$ & $\begin{array}{l}118 \\
113 \\
108 \\
106 \\
105 \\
65 \\
42\end{array}$ & $\begin{array}{l}51 \\
50 \\
50 \\
49^{*} \\
47 \\
34 \\
25\end{array}$ & $\begin{array}{l}40 \\
39^{*} \\
39^{*} \\
38^{*}\end{array}$ & $\begin{array}{l}29 \\
29 \\
29 \\
29 \\
27.5\end{array}$ \\
\hline$A_{s}(V)$ & $\begin{array}{l}0 \\
0.1 \\
0.2 \\
0.5 \\
1 \\
4 \\
8\end{array}$ & $\begin{array}{c}200 \\
80 \\
55 \\
(150-240) \\
(2185) \\
80\end{array}$ & $\begin{array}{c}- \\
- \\
\overline{4} \\
4.5 \\
19 \\
(2110) \\
60\end{array}$ & $\begin{array}{c}\overline{-} \\
- \\
\overline{-} \\
\bar{n} \\
(80-140) \\
n 64\end{array}$ & $\begin{array}{c}- \\
- \\
- \\
- \\
(14,65)^{\circ} \\
53\end{array}$ & $\begin{array}{c}- \\
\overline{-} \\
\overline{-} \\
\overline{(14,53)} \\
38\end{array}$ & $\begin{array}{c}- \\
= \\
(81-20) \\
{ }_{21}^{4} \\
18\end{array}$ & $\begin{array}{l}- \\
- \\
- \\
11 \\
16 \\
13\end{array}$ & $\begin{array}{l}- \\
- \\
12 \\
12 \\
10\end{array}$ & & $\begin{array}{c}017 \\
- \\
10.5\end{array}$ \\
\hline$B(I I I)$ & $\begin{array}{l}0 \\
0.1 \\
0.2 \\
0.5 \\
1 \\
4 \\
8\end{array}$ & $\begin{array}{l}\text { ads } \\
\text { ade } \\
\text { ado } \\
\text { ads } \\
162 \\
46\end{array}$ & $\begin{array}{l}a^{-} \\
\text {ade" } \\
\text { ads" } \\
\text { ads" } \\
110 \\
45\end{array}$ & $\begin{array}{l}\text { ads" } \\
2235 \\
2220^{\star} \\
200 \\
85 \\
45\end{array}$ & $\begin{array}{r}- \\
2125 \\
115 * \\
2100 \\
46 \\
34\end{array}$ & $\begin{array}{l}-\overline{70} \\
70 \\
67^{\star} \\
60 \\
34 \\
22\end{array}$ & $\begin{array}{l}-\bar{t}^{\star} \\
21 \\
19.5^{\star} \\
18 \\
14 \\
12.5\end{array}$ & $\begin{array}{l}-7 \\
9 \\
9 \\
9 \\
9 \\
9 \\
8.3\end{array}$ & $\begin{array}{c}0 \\
7 \\
7 \\
7 \\
27 \\
6\end{array}$ & $-*$ & $\begin{array}{l}7.5 \\
7.5 \\
7.5 \\
4.2\end{array}$ \\
\hline$B(I I)$ & $\begin{array}{l}0 \\
0.1 \\
0.2 \\
0.5 \\
1 \\
4 \\
8\end{array}$ & $\begin{array}{r}\text { ado } \\
\text { ade } \\
420 \\
250 \\
20 \\
7\end{array}$ & $\begin{array}{l}-17 \\
01 \\
7 \\
10 \\
11 \\
11\end{array}$ & $\begin{array}{l}-5 \\
219 \\
11 \\
24 \\
6 \\
21\end{array}$ & $\begin{array}{c}- \\
\bullet 11\end{array}$ & $\begin{array}{l}- \\
- \\
= \\
- \\
-\end{array}$ & $\begin{array}{l}= \\
= \\
= \\
=\end{array}$ & $\begin{array}{l}= \\
= \\
= \\
=\end{array}$ & $\begin{array}{l}=* \\
=* \\
=\end{array}$ & & \\
\hline
\end{tabular}

\footnotetext{
an mobere zoprenent two diatinct peake.
} 
Table A-3. Distribution Coefficlents on Dowex $1 \times 10$ Anton-Exchange Resin (200-400 mesh, chloride form) as a Punction of HCl Concentration (concentrations in molarity)

ada: adsorbed

s1: slight

-: not adsorbed

*: assigned value

( ): Indefinite elution curve, talling, poor reproducibility

ppt: precipitate

HCl

\begin{tabular}{|c|c|c|c|c|c|c|c|c|c|}
\hline Blewent & 0.1 & 0.2 & 0.5 & 1 & 3 & 6 & 9 & 10 & 12 \\
\hline$T 1(I V)$ & - & - & - & - & - & - & - & 24 & 55 \\
\hline $\mathrm{Zr}(I V)$ & - & - & - & - & - & - & $(81-30)$ & ads & ads \\
\hline Hf(IV) & - & - & - & - & - & - & 24 & $(10-80)$ & ads \\
\hline Sn(IV) & ppt & (ppt?) & 315 & ads & ads & ads & ads & ads & \\
\hline Mo(VI) & $(80-200)$ & $(25-100)$ & $(15-70)$ & $(5-50)$ & 98 & 210 & 153 & 115 & $(150-250)$ \\
\hline $\operatorname{Re}$ (VII) & & ads & ads & ads & 380 & 81 & & $\begin{array}{l}16.5 \\
2120\end{array}$ & 26 \\
\hline $\begin{array}{l}\text { 日g(II) } \\
\mathrm{Pd}(\mathrm{II})\end{array}$ & ads & ads & & $\begin{array}{l}\text { ads } \\
\text { ads }\end{array}$ & $\begin{array}{r}\text { ada } \\
\sim 500\end{array}$ & $\begin{array}{l}\text { ads } \\
120\end{array}$ & $\begin{array}{r}2200 \\
55\end{array}$ & $\begin{array}{r}2120 \\
38\end{array}$ & $\begin{array}{l}58 \\
28\end{array}$ \\
\hline BI (III) & ppt & ads & ads & ads & ads & 202 & 74 & 57 & $\begin{array}{l}20 \\
43\end{array}$ \\
\hline $\mathrm{Pb}(\mathrm{II})$ & - & 11 & 19 & 24 & 15 & 23 & - & - & - \\
\hline In(III) & - & $81 ?$ & 81 & 10 & 20 & 18 & 13 & 11. & 7.5 \\
\hline$\nabla$ & - & - & - & - & - & - & - & $-*$ & - \\
\hline$U(V I)$ & - & - & - & 21.2 & 35 & ads & ads & ads & ads \\
\hline $\mathrm{Te}$ (IV) & - & - & - & - & 730 & ads & ads & ade & ads \\
\hline$T e(V I)$ & - & - & - & - & & & & & \\
\hline Fo(III) & - & - & - & थ1.5 & (45) & ads & ads & ads & ads \\
\hline $\operatorname{co}(I I)$ & - & - & - & - & - & 9 & 71 & 64 & 45 \\
\hline G(III) & - & - & - & 32 & $(81-90)$ & ads & ads & ada & ads \\
\hline $\operatorname{Cd}(\mathrm{II})$ & 400 & ads & ads & ads & ads & 2370 & 68 & 36 & 15 \\
\hline $2 n(I I)$ & 9 & 25 & 92 & ads & ads & 245 & 90 & 68 & 45 \\
\hline $\boldsymbol{M}(\mathbf{v})$ & - & - & - & - & - & - & - & - & - \\
\hline B(III) & - & - & - & - & - & - & - & - & - \\
\hline$A$ (III) & - & - & - & - & - & - & - & & - \\
\hline$B \in(I I)$ & - & - & - & - & - & - & - & & - \\
\hline $8 b(v)$ & ppt & ppt & Ppt & pt?)- & & & & & \\
\hline $8 e$ (III) & 1 & - & - & - & - & - & $=$ & & - \\
\hline$P(\nabla)$ & - & - & - & - & - & - & $=$ & $\because$ & - \\
\hline$G$ (III) & - & - & - & - & - & - & - & - & \\
\hline $\sin (\mathrm{II})$ & - & - & - & - & $-1 ?$ & $-1 ?$ & 2.8 & & 3.8 \\
\hline 48 (II) & - & - & - & - & - & - & - & & $=$ \\
\hline $8 x(I I)$ & - & - & - & - & - & - & - & & - \\
\hline $\mathrm{Ba}(I I)$ & - & - & - & - & - & - & - & - & - \\
\hline$M(I I)$ & - & - & - & - & - & - & - & & - \\
\hline
\end{tabular}


Tab1e A-4. Dietribution Coctficiente on Dowex 1x4 Anton-Bxchenge Beain (100-200 mesh, chloride form) as a Function of BC1 Concentration (concentration in molerity)

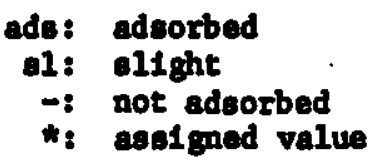

( ): Indefinite elution curve, talling, poor reproduclbility ppt: precipltate

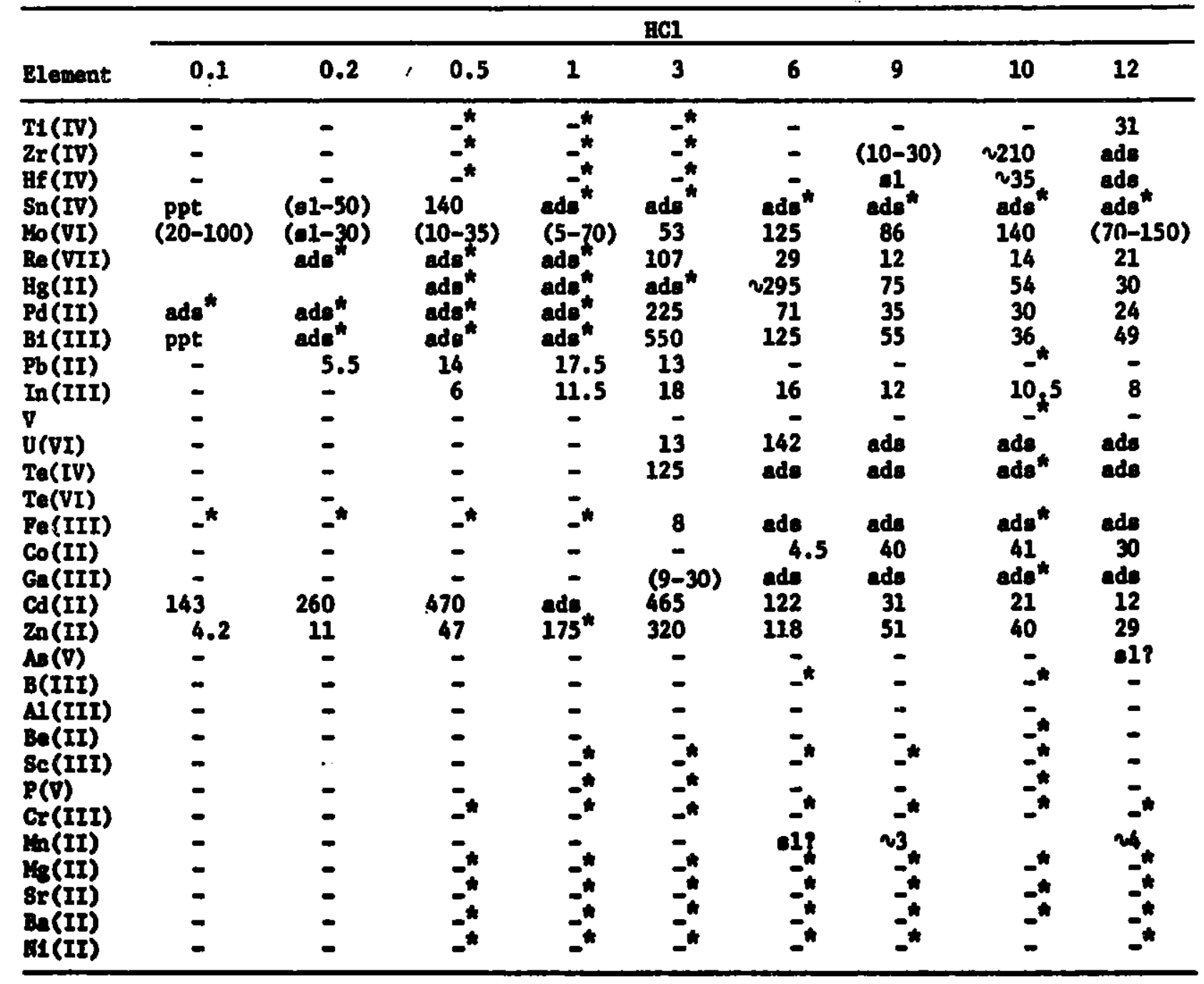


Table A-5. Distribution Coefficiente on Dowex 1x10 An1on-Exchange Resin (100-200 mesh, fluoride form) as a Function of HF Concentration (concentrations in molarity)

ads: adsorbed

al: slight

-: not adsorbed

*: assigned value
( ): Indefinite elution curve, talling, poor reproductbility

ppt: precipitate

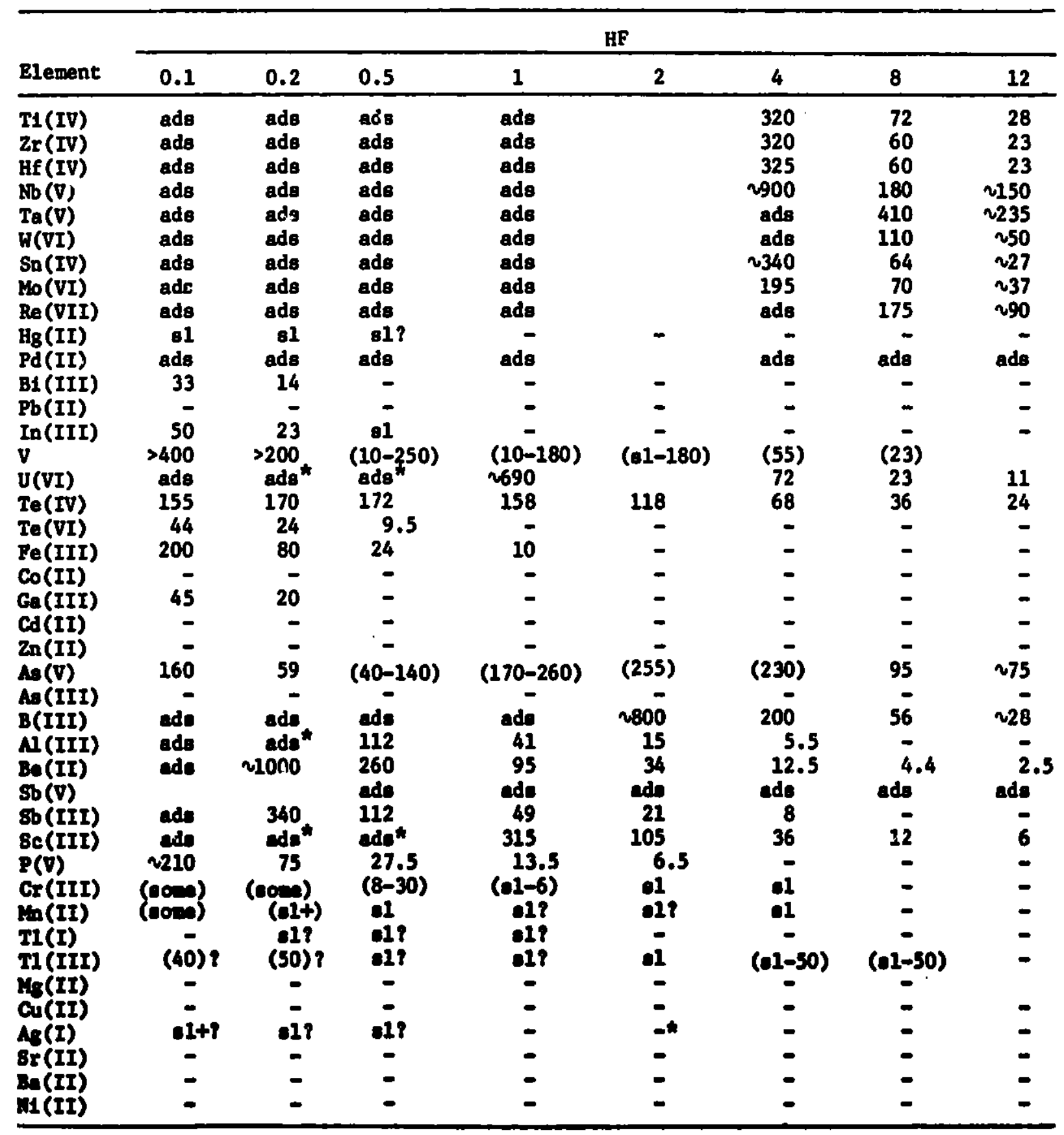


Table A-6. Diatribution Coefficlents on Dowex $1 \times 4$ Anton-Exchange Resin (100-200 mesb, fluoride form) as a Function of HF Concentration (concentrations in molarity)

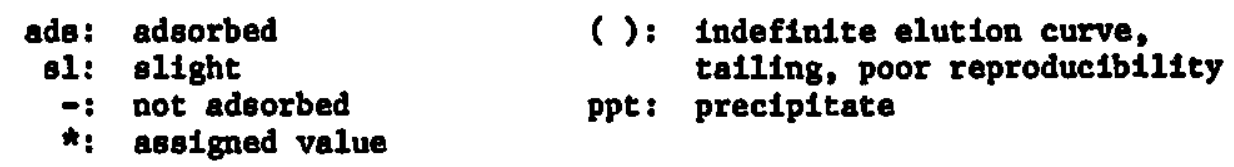

( ): Indefinite elution curve, talling, poor reproduclbility ppt: precipitate

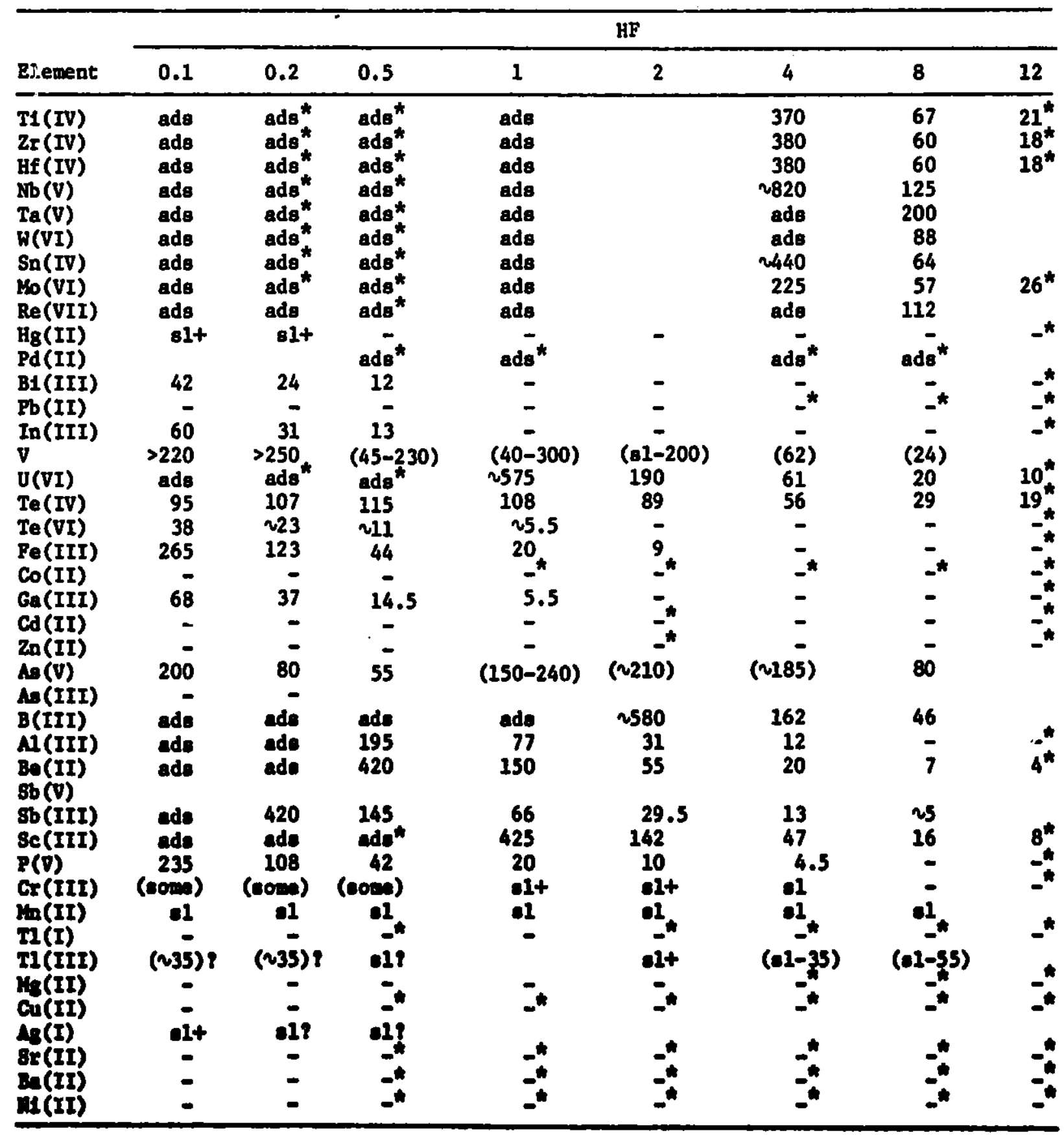


Table A-7. Distribution Coefficients for Several Elements in Dilute Hydrofluoric Acid (Dowex anion-exchange resin, 200-400 mesh and 100-200 mesh, fluoride form; HF concentrations in molarity)

\begin{tabular}{|c|c|c|c|c|c|c|c|c|}
\hline \multirow[b]{2}{*}{ Element } & \multicolumn{8}{|c|}{ Distribution Coefficient at Indicated HF Concentration } \\
\hline & 0.1 & 0.15 & 0.2 & 0.3 & 0.4 & 0.5 & 0.6 & 1.0 \\
\hline & \multicolumn{8}{|c|}{ Dowex $1 \times 10,200-400$ Mesh } \\
\hline \multirow[t]{2}{*}{$\begin{array}{l}\mathrm{BI} \text { (III) } \\
\mathrm{Ga} \text { (III) } \\
\mathrm{In} \text { (III) } \\
\mathrm{Fe} \text { (III) } \\
\mathrm{P}(\mathrm{V})\end{array}$} & $\begin{array}{r}33 \\
45 \\
50 \\
200 \\
2210\end{array}$ & $\begin{array}{r}17 \\
26 \\
32 \\
112 \\
103\end{array}$ & $\begin{array}{l}14 \\
20 \\
23 \\
80 \\
75\end{array}$ & $\begin{array}{l}10.8 \\
13 \\
14 \\
46 \\
48\end{array}$ & $\begin{array}{l}- \\
w 6 \\
w 9 \\
32 \\
34\end{array}$ & $\begin{array}{c}- \\
- \\
81 \\
24 \\
27.5\end{array}$ & & $\begin{array}{l}- \\
- \\
\overline{10} \\
13.5\end{array}$ \\
\hline & \multicolumn{8}{|c|}{ Dowex $1 \times 4,100-200$ Megh } \\
\hline $\begin{array}{l}\mathrm{BI} \text { (III) } \\
\mathrm{Ga} \text { (III) } \\
\text { In (III) } \\
\mathrm{Fe} \text { (III) } \\
\mathrm{P} \text { (V) }\end{array}$ & $\begin{array}{r}42 \\
68 \\
60 \\
265 \\
235\end{array}$ & $\begin{array}{r}31 \\
49 \\
42 \\
185 \\
155\end{array}$ & $\begin{array}{r}24 \\
37 \\
31 \\
123 \\
108\end{array}$ & $\begin{array}{l}17 \\
25 \\
21 \\
80 \\
68\end{array}$ & $\begin{array}{l}14 \\
18 \\
16 \\
57 \\
51\end{array}$ & $\begin{array}{l}12 \\
14.5 \\
13 \\
44 \\
42\end{array}$ & $\begin{array}{l}10.7 \\
12.5 \\
10.5 \\
35 \\
34\end{array}$ & $\begin{array}{l}- \\
5.5 \\
\overline{20} \\
20\end{array}$ \\
\hline
\end{tabular}




\section{BIBLIOGRAPHY}

1. A. A. Abde1-Rassoul, H. F. Aly, and A. B1-Haggan, Interseparation of Bafnium-Z1rcontum from Uranlum Matr1x, Radiochem. Radioanal. Lett. 18, 267 (1974); Nucl. Sc1. Abstr. 30, 23588 (1974); Anal. Abstr. 28, 4B72 (1975).

2. W. H. Adams, J. R. Buchholz, C. W. Chrlstenson, G. I. Johnson, and B. B. Fowler, Studies of Plutonium, Americium, and Uranium in Environmental Matr1ces, LA-5661 (1975); Chem. Abstr. 83, 3899 (1975).

3. R. Albert, M. Cuypers, A. LesbatB, and E. Mlgnonsin, New Developments in the Systemat1c Analyeis of High Purfty Metals and Bspectally of Aluminum, Copper and Zirconium, Proc. Conf. On Modern Trends In Activation Analysis, College Sta., Texas, 1965, p. 310.

4. G. Alberti, V. Caglioti, and M. Lederer, Chromatography on Paper Impregnated with Ion Exchange Resins, $V$. The Study of Complex Equilibria, J. Chromatogr. 7, 242 (1962); Nucl. Scl. Abstr. 16, 11548 (1962).

5. A. Allan and W. Sanad, Analogy between Amine Extraction of Metals and Their Anlon-Exchange Sorption on Solid Resins, Microchem. J. 12, 418 (1967); Nuc1. Sc1. Ab8tr. 21, 45224 (1967).

6. 0. V. Al'tshuler and E. A. Subbotina, II. Removal of Traces of Niobium and Titanium from Tantalum by Ion Exchange, Russ. J. Inorg. Chem. 4 , 11 (1959); [Trans1. of $\mathrm{Zh}$. Neorg. KhIm. 4, 28 (1959)]; Chem. Abstr. 53, 12972 (1959).

7. 0. V. Al'tshuler, B. A. Subbotina, and A. F. Afanas'eva, Separation of N1oblum and Titantum by the Method of Ion-Bxchange, Rus8. J. Inorg. Chem. 3(5), 175 (1958); [Trans1. of Zh. Neorg. KhIm. 3, 1192 (1958)]; Chem. $\overline{A b s t r}$. 53, 12085 (1959); Nuc1. Sc1. Abstr. 13, 3638 (1959).

8. American Society for Testing and Materials, Chemical Analygis of Titanium and Titantum-Base Alloys, B 120-69, 1971 Book of ASTM Standards, Part 32, ASTM, PhIladelphia, Pa., p. 554 (1971).

9. Ibid., Chemical Analygis of Zircontum and Zirconium-Base Alloys, B 146-68, p. 631 .

10. Ib1d., Chentcal Analyels of Berrocolumblum (Tentative Yathod), B 367-70T, p. 947 .

L1. M. S. Ander8on, Determination of Ta-182 by Ion Brehange, Ann. Prog. Report of Diviston Analyt1cal Branch for 1964, DD0-14655, p. 201 (1965); Kucl. 8c1. Abstr. 19, 32059 (1965).

12. I. I. Angelov, V. 8. Hechaeva, and A. A. Zakeev, States of Areentc In solutions of Bydrofluortc Ac1d, Tr. Vees. Mauchno.-Iseled. Int. Whim. Reakt. Osobo Chist. Khim. Veshchestv. 29, 155 (1966), (Rusalan); Chem. Abstr. 67, 57679 (1967). 
13. E. Antal, E. Szabo, A. Simonfts, and P. Quittner, Determination of Sodium and Potassium in Tungsten by Neutron Activation Analys1s, Ratiochem. Conf. Abstr. Papers, Brat1slova, 1966, p. 79; Chem. Abstr. 68, 56312 (1968).

14. J. W. Arden and J. W. McMillan, Determination of Zirconfum-95 in Graphite from H.T.G.C. Loops; Anion-Exchange Separation of Zirconium-95 from Scandlum-46 and Iron-59, AERE-R 4872 (1965); Anal. Abstr. 12, 6420 (1965); Nuc1. Sci. Abstr. 19, 26224 (1965).

15. M. Artel and E. Kirowa, The Anion-Exchange Separation of Tin, Antimony, Lead and Copper, Talanta 8, 214 (1961); Chem. Abstr. 56, 921 (1962).

16. P. I. Artyukhin, E. N. GII'bert, and V. A. Pronin, Activation Determination of Impuritles in Gallium, Izv. Stb. Otd. Akad. Nauk SSSR, Ser. Kh1m. Nauk, 1966(3), 138 (1966), (Russian); Anal, Abstr. 14, 3874 (1967); Chem. Abstr. 65, 9715 (1966).

17. P. I. Artyukhin, E. N. Gil'bert, and V. A. Pronin, Radioactivation Determination of Impurities in Antimony, J. Anal. Chem. 21, 452 (1966); [Trans1. of $\mathrm{Zh}$. Anal. KhIm. 21, 504 (1966)]; Anal. Abstr. 15, 1354 (1968); Nucl. Sc1. Abstr. 20, $2 \overline{6814}$ (1966).

18. P. I. Artyukhin, E. N. Gil'bert, and V. A. Pronin, Determination of Impurities in Iron by Neutron Activation, J. Anal. Chem. USSR 22, 92 (1967); [Transl. of Zh. Anal. KhIm. 22, 111 (1967)]; Nucl. Sc1. Abstr. 21, 12362 (1967), 23, 26199 (1969); Chem. Abstr. 67, 7695 (1967).

19. P. I. Artyukhin, E. N. G11'bert, and V. A. Pronin, ActIvation Determination of Impurities in Some Semiconductor Materlals, Tr. Kom. Anal. Khim. Akad. Nauk SSSR, Inst. Geokhim. Anal. Kh1m. 16, 169 (1968), (Russian); Chem. Abstr. 69, 56701 (1968).

20. V. T. Athavale, M. N. Nadkarn1, and C. H. Venkateswarlu, Ion-Exchange Separation of Titanium from Steels and Its De:ermination with Hydrogen Peroxide, Anal. Chim. Acta 23, 438 (1961); Chem. Abstr. 55, 1276 (1961).

21. V. T. Athavale, P. P. Parekh, and M. Sankar Das, Studies on the Radioactive Disequilibrium of Certain Uranium Bearing Ores of India: Part I - Isolation and Est imation of Uranium and Its Daughter Products, Indian J. Chem. 6, 206 (1968); Chem. Abstr. 69, 63893 (1968).

22. G. Aubouin, Activation Analysis of Mineral Impurities in Terphenyls and Their Determination by Gamma-Spectrometry, Radlochim. Acta 1, 117 (1963), (French); Nuc1. Sc1. Abstr. 17, 28813 (1963).

23. G. Aubouin, H. Dabrowsk1, J. Laverlochere, and J. V1al, Deatructive and Nondestructive Analysis of Solls and Minerals by Neutron Activation, CEA-CONF-1183 (1968), (French); Nuc1. Sc1. Abstr. 23, 13671 (1969); Chem. Abstr. 71, 35644 (1969).

24. G. Aubouin, J. Diebolt, E. Junod, and J. Laverlochere, Some Applications of Ion-Exchange Rasins in Activation Microanalysis, Proc. Conf. on Wodern Trends In Activation Analysis, College Station, Texas, 1965, p. 344. 
25. G. Aubouin, F. Mugain, and J. Laverlochere, Determination of Impurities In Tantalum and Niobium by Neutron Radioactivation, Bul1. Soc. Chim. Fr. (2), 547 (1965), (French); Chem. Abstr. 62, 15406 (1965); Nuc1. Sc1. Abstr. 19, 24416 (1965).

26. G. Aubouin and J. Laverlochere, Ion Exchange Resin Separations Applied to Radioactivation Analysis, CEA-2359 (1963), (French); Anal. Abstr. 13, 2935 (1966); Chem. Libstr. 60, 13856 (1964).

27. G. Aubouin and J. Laverlochere, Improvement of Chemfcal Separations on Ion Exchange Resins: Influence of Particle Size, J. Radioanal. Chem. 1. 123 (1968), (French); Chem. Abstr. 68, 111019 (1968); UCLR-TRANS-10324 (1968); Nuc1. Sc1. Abstr. 23, 17521 (1)

28. G. Bagliano and L. Ossicln1, The Adsorption of Metal Ions from HF-HC10 Mixtures on Some Ion Exchange Papers, J. Chromatogr. 21, 498 (1965); Chem. Abstr. 64, 16679 (1966).

29. V. I. Baranovskil and M. R. Nikftin, Study of Ion Exchange In HF Solutions. Non-Exchange Sorption of Hydrofluoric Acld by Ion Exchangers, Collo1d J. USSR 26, 126 (1967); [Trans1. of Kolloldn. Zh. 26, 153 (1964)]; Chem. Abstr. 61, 3739 (1964).

30. M. K. Barnett, The Concentration of Protactintum by Re-Cycling Through an Anion-Exchange Column, J. Inorg. Nucl. Chem. 4, 358 (1957); Chem. Abstr. 51, 16173 (1957).

31. D. L. Barney and C. E. Kent, Ion Exchange Separation Processes for Nioblum and Tantalum, Ind. Eng. Chem. Process Des. Dev. 7, 1 (1968); Nuc1. Sc1. Abstr. 22, 6940 (1968).

32. E. R. Bautista and G. Dominguez, Radioanalytical Purification of Protactinum by Anton Exchange In Hydrofluoric AcId - Sulfuric Actd Medium, An. Quim. 67, 375 (1971), (Spanish); Nucl. Sc1. Abstr. 26, 33121 (1972).

33. N. V. Bausova and G. A. Reshetnikova, Sorption of Fluoride Complexes of Tantalum, Nioblum, Titanium, and Vanadium(V) on Anion Exchanger AV-17 from Aqueous Acetone Solutions, Tr. Inst. Khim. Akad, Nauk SSSR, Ural. F11. (17), 201 (1970), (Russian); Chem. Abstr. 74, 25362 (1971).

34. R. K. Bell and 0 . Menis, Ion Exchange Separation of Mo-W in the SIntered Compact, Annu. Rept., 1965-66, NBS-Ti-402, p. 24 (1967).

35. P. A. Benson, W. D. Holland, and R. H. Smlth, Determination of Iron and Uranium In High Purity Lead Foil by Neutrojn Activation Analysis, Anal. Chem. 34, 1113 (1962); Proc. Conf. on Modern Trends In Activation Analysis, College Station, Texas, 1961, p. 7; Chem. Abstr. 57, 7895 (1962).

36. K. S. Bergstresser, Spectrophotonetric Determination of Klobium in Tantalum, Anal. Chem. 31, 1812 (1959). 
37. G. Beurton, B. Beyssier, and Y. Angella, Determination of About Th1rty Elements in Aluminum and Its Alloys by Means of Neutron Activation Analysis, J. Radioanal. Chem. 38, 257 (1977).

38. T. R. Bhat and Y. W. Gokhale, Anton-Exchange Study of the Uranyl Fluorine Complexes, J. Sc1. Ind. Research (Ind1a) 18B, 532 (1959); Nuc1. Sc1. Abstr. 14, 9428 (1960).

39. E. J. Bieleck1, Use of Ion-Exchange Techniqueg for Production of H1ghPurfty Potassium Fluorotantalate, Proc. Conf. on Advances in Extractive Metallurgy, Inst. Mining and Metallurgy, London, 1968, p. 776; Nuc1. Sc1. Abstr. 24, 295 (1970).

40. E. J. Bieleck1, C. E. Mosheim, and G. J. Nessle, Use of Ion Exchange for Production and Recovery of High-Purity Potassium Flurotantalate, TMS Paper No. A71-57, Am. Inst. of Mining, Metallurglcal and Petroleum Eng., New York (1971;; Nucl. Sc1. Abstr. 26, 20032 (1972).

41. T. H. B1shay, Application of Radioactlvation to the Sequential Separation of Antimony, Cadmium, Chromium, Cobalt, Iron, Tin, and Zinc from Aluminum and Lead by Ion-Exchange Chromatography, Anal. Chem. 44, 1087 (1972).

42. S. Bjornholm and L. Westgaard, Preparation of Curle Amounts of ${ }^{234} \mathrm{Th}\left(\mathrm{UX}_{1}\right.$ ) and Millicuries of ${ }^{234} \mathrm{~Pa}(\mathrm{UZ})$ by Extraction of ${ }^{238} \mathrm{U}$, Radiochim. Acta 8 , 204 (1968); Nuc1. Sc1. Abstr. 22, 33382 (1968).

43. J. Blachot, A. Benabed, J. Herment, and E. Monnand, Radiochemical Separations of Hafnium, Tantalum and Germanium from Tungsten and Selenium Irradiated by 14-MeV Neutrors, CEA-R-3678 (1968), (French); Nuc1. Sc1. Abstr. 23, 21775 (1969); Chem. Abstr. 71, 8770 (1969); ORNL-tr-2390 (1970) (English Trans.).

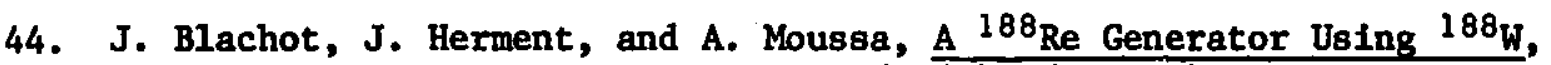
Int. J. App1. Radiat. Isot. 20, 467 (1969), (French); Nuc1. Sc1. Abstr. 23, 31382 (1.969).

45. N. N. Bocharova, G. N. Razumova, and I. D. Shuba, Neutron Activation Method of Determining Trace Impurities of $\mathrm{Au}, \mathrm{Cu}$, and $\mathrm{Zn}$ In Mercury Teliurlde and in the Ternary Compound $\mathrm{Cs}_{\mathrm{H}} \mathrm{H}_{3-\mathrm{Te}}$, Sov. Radiochem. 14, 165 (1972); [Trans1. of Rad1okhimfya 14, 158 (1972)]; Nuc1. Sc1. Abstr. 26, 50227 (1972).

46. C. Bondy, Application of Various Methods In Activation Analysis for Iron and Cobalt, Anal. Chim. Acta 38, 579 (1967), (French); Chem. Abstr. 67, 60625 (1967).

47. A. L. Bonl, Quantitative Analysis of Radionuclides in Process and Environmental Samples, Anal. Chem. 32, 599 (1960).

48. M. C. de Bortoll, Radiochemicel Determination of Plutonium In Sol1 and Other Bnvironmental Samples, Anal. Chem. 39, 375 (1967). 
49. R. F. Brigevich and R. A. Kuznetsov, Isolation of Radloactive Phosphorous from Irradiated Sulfur, Sov. Radiochem. 10, 475 (1968); [Trans1. of RadiokhImiya 10, 487 (1968)]; Chem. Abstr. 69, 92096 (1968).

50. U. A. Th. Brinkman, P. J. J. Sterrenburg and G. de Vries; Chromatographic Techniques using Liquid Anton Exchangers, IV. HF Systems, J. Chromatogr. 54,449 (1971); Anal. Abstr. 21, 3985 (1971).

51. J. K. Brody, Spectrochemical Research in an Atomic Energy Laboratory, IX. Colloquim Spectroscopicum Internationale, Vo1. 1, Pub1. G.A.M.S., Par1s, p. 231 (1961).

52. F. I. Brouchek and Ts. N. Gudushaur1, Study of the Desorption of Niobtum from the Anion Exchangers of Fluoroforms, Soobshch. Akad. Nauk Gruz. SSSR 70, 85 (1973), (Russian); Nuc1. Sc1. Abstr. 28, 7891 (1973).

53. F. I. Brouchek and Ts. N. Gudushaur1, Adsorption of Titanium, Tungsten, Molybdenum and Vanadium from Tartrate Medium on the Fluoride Forms of Anlonites AV-16 and AV-17, Tr. Gruz. Politekh. Inst. 4(177), 57 (1975), (Russian); Anal. Abstr. 32, 2B16 (1977).

54. F. I. Brouchek and Ts. N. Gudushauri, Desorption of Titanium, Tungsten, Molybdenum and Vanadium from the Fluoride Forms of Anionites AV-16 and AV-17, Tr. Gruz. Polltekh. Inst. 4(177), 60 (1975), (Russtan); Anal. Abstr. 32, 2B17 (1977).

55. M. N. Bukhsh, J. Flegenhelmer, F. M. Ha11, A. G. Maddock, and C. Ferre1ra de Miranda, The Chemistry of ProtactInium, VII. The Fluoro-Complexes, J. Inorg. Nuc1. Chem. 28, 421 (1966); Nuc1. Sc1. Abstr. 20, 18681 (1966); Physicr-Chemfe du Protactinium, Proc. 1965 Orsay Conf., Centre Nat. de la Rech. Sc1., Paris, p. 195 (1966); Nucl. Sc1. Abstr. 21, 8313 (1967).

56. R. M. Burd, G. W. Goward, M. D. Hartman, M. A. McCracken, and B. B. W1lson, The Determination of Aluminum as an Impurity in Zirconium and Zircaloy, WAPD-C(AR)-143 (1957); Chem. Abstr. 54, 20646 (1960); Nucl. Sc1. Abstr. 13, 13242 (1959).

57. G. A. Burney, Ion Bxchange Process for the Recovery of $\mathrm{Pu}^{238}$ from Irradlated Np237, Ind. Eng. Chem. Process Des, Dev. 3, 328 (1964); Chem. Abstr. 61, 11566 (1964).

58. Yu. A. Buslaev and N. S. Nkolaev, The $\mathrm{HF}-\mathrm{TaF}_{2}-\mathrm{H}_{2} \mathrm{O}$ System, Russ. J. Inorg. Chem. 4, 210 (1959); [Transl. Zh. Neorg. Khim. 4, 465 (1959)]; Chem. Abstr. 53, 15739 (1959).

59. J. F. Byrum, Anton Exchange from BF Media, MIT Prog. Rept. NY0-2668, p. 20 (1961); Nuc1. Sc1. Abstr. 16, 2664i (1962).

60. M. J. Cabell and I. Milner, The Preparation of Tantalum-Free Mlobium Pentoxdde, J. Appl. Chem. 5, 482 (1955).

61. M. J. Cabell and I. Milner, The Quantitative Separation and Determination of Tantalum and Miobium using Anion Exchange Chromatography, Ans1. Chim. Acta 13, 258 (1955); Nuc1. Sc1. Abstr. 9, 7703 (1955); Chem. Abstr. 50, 16550 (1956). 
62. A. Cassol, Methy1 TIn(IV) Fluoride Complexes. Anton-Exchange and Potentiometric Studies, Gazz. Chim. Ital. 96, 1764 (1966), (Itallan); Chem. Abstr. 67, 15436 (1967).

63. A. Cassol, L. Magon, and R. Basbier1, Complexes of Organometallic Compounds, IX. Invest1gations on Organotin-Fluor1de Systems by Means of Anion Exchange Paper Chromatography and Paper Electrophoresis, J. Chromatogr. 19, 57 (1965); Chem. Abstr. 63, 12356 (1965).

64. A. Cassol and R. Portanova, Dimethyl Tin(IV) Fluoride Complexes. AnionExchange and Potentiometric Studies, Gazz. Chim. Ital. 96, 1734 (1966), (Italian); Chem. Abstr. 67, 15434 (1967).

65. A. Cazaussus, H. Arapaki-Strapelias, and R. Muxart, Behavior of Protactinium(V) According to Indicator Scale, in Aqueous Alkaline Solutions, Radiochem. Radioanal. Lett. 6, 297 (1971), (French); Chem. Abstr. 75, 58185 (1971).

66. B. R. Chamberlain and R. J. Leech, Determination of Microgram Quant1t1e8 of TIn (IV) by a Combined Ion-Exchange/X-Ray Fluorescence Technique, Talanta 14, 597 (1967); Chem. Abstr. 67, 17576 (1967).

67. K. M. Chan and J. P. RAley, Determination of Tungsten In S1licates and Natural Waters, Anal. Chim. Acta 39, 103 (1967); Chem. Abstr. 67, 96578 (1967).

68. F. W. Chapman, Jr., G. G. Marvin, and S. Young Tyree, Jr., Volat1lization of Elements from Perchloric and Hydrofluoric Acld Solutions, Anal. Chem. 21, 700 (1949).

69. S. M. Chernobrov and N. P. Kolonina, Separation of Nloblum, Tantalum and TItanium on Anion-Exchange Resins, Khromatog., ee Teoriya 1 Primenenie, Akad. Nauk SSSR, Trudy Vsesoyuz.-Soveshchaniya, Moscow, 1958, p. 214 (1960), (Russian); Chem. Abstr. 55, 24384 (1961); Anal. Abstr. 8, 4596 (1961).

70. A. Chetham-Strode, Jr. and 0. L. Keller, Jr, Ion Exchange of Protactinium(V) In HP Solutions and Some Appilcatinns, Physico-Chemie du Protactinium, Proc. Orsay 1965 Conf., Centre Nat. de la Rech. Sc1., Par18, p. 189 (1966); Nucl. Sc1. Abstr. 21, 8418 (1967).

71. J. M. Chilton, Observations on the Behavior of Protactinium in HC1-HF Solutions, Proc. Protactinium Chem. Symp., Gatlinburg, Tenn., 1963, TID-7675 (1964); Nuc1. Sc1. Abstr. 18, 10049 (1964); Chem. Abstr. 60, 13928 (1964).

72. G. R. Choppin and T. Sikkeland, Scheme for the Separation of the Elements Franctum through Urantum, The Radiochemistry of Thorfun, . $_{\text {. }}$. Hyde, NAS-NS 3004, p. 60 (1960); Nucl. Sc1. Abstr. 14, 10499 (1960).

73. N. Y. Chu, Plutonlum Determination In Soll by Leaching and Ion-Exchange Separation, Anal. Chem. 43, 449 (1971). 
74. D. A. Collins, J. J. Hillary, J. S. Nairn, and G. M. PhIllips, The Development and Application of a Process for the Recovery of Over $100 \mathrm{~g}$ of Protactinium-231 from a Uranium Refinery Waste Material, J. Inorg. Nuc1. Chem. 24, 441 (1962); Nuc1. Sc1. Abstr. 17, 4632 (1963).

75. M. Csajka, M. Ordogh, and E. Szabo, Determination of Contaminants (Arsenic, Copper, Zinc, Antimony and Bismuth) in Nickel of Graded Purity, Acta Chim. Acad. Sc1. Hung. 51, 161 (1967); Anal. Abstr. 15, 2652 (1968).

76. A. R. Dale and P. A. Jones, Study of the Estimation of Certain Trace Impuritles by Fluorimetrlc Methods, the J. A. Radley Res. Inst. Final Tech. Rept. AD-467884 (1965).

77. R. Dams and J. Hoste, Neutron ActIvation of Traces in Electrolyt1c Zinc. Part I. SImultaneous Determination of Mercury; Uranium and Yttrium, Anal. ChIm. Acta 39, 423 (1967); Chem. Abstr. 67, 104868 (1967); Nuc1. Sci. Abstr. $21,4 \overline{0790}$ (1967).

78. L. Danielsson, Adsorption of a Number of Elements from $\mathrm{HNO}_{3}-\mathrm{HF}$ nnd $\mathrm{H}_{2} \mathrm{SO}_{4}-\mathrm{HF}$ Solutions by Cation and Anfon Exchange, Acta Chem. Scand. 19, 1859 (1965); Chem. Abstr. 64, 10441 (1966).

79. L. Danielsson, Separation of Traces of Elements from Large Amounts of Iron by Anton Exchange, Ark. Reml 27, 453 (1967); Nucl. Sc1. Abstr. 22, 10328 (1968).

80. L. Danielsson, Anion Exchange Study of Some Elements Strongly Adsorbed from Sulphurlc-Bydrofluorle Ac1d Mixtures, Ark. Reml 27, 459 (1967); Nucl. Sc1. Abstr. 22, 10336 (1968).

81. L. Danielsson, Studies of Ion Exchange Separations with Particular Reference to the Analysis of Iron and Steel, Ark. Kem1 27, 467 (1967); Chem. Abstr. 67, 121923 (1967).

82. I. Danielsson, Colorimetric Determination of Small Amounts of Nioblum In Steel after Separation by AnIon Exchange, Jernkontorets Ann. 151, 325 (1967); Muc1. Sc1. Abstr. 22, 1979 (1968); Chem. Abstr. 68, 45953 (1968).

83. F. De Corte, Activation Analysis of Trace Impurities in Silicon, Thesis, State Univ. of Ghent (1970); Nucl. Sc1. Abstr. 25, 15547 (1971).

84. F. De Corte, A. Speecke, and J. Hoste, Activation Analysis of High-Purtty S111con, II. Multi-Blement Analysis: Separation Scheme for 21 Elements, J. Badioane1. Chea. 8, 287 (1971); Mucl. Sc1. Abstr. 26, 11555 (1972).

85. J. P. De Kle1jn and B. Van Zanten, 18 F-Radiometric Determination of the Fuoride Content of an Anton Brchange Redin In the Fluoride Form, J. Badionnal. Chen. 35, 207 (1977).

86. J. P. Da Kleijn and B. Van zanten, The Fluoride Content of an Anton

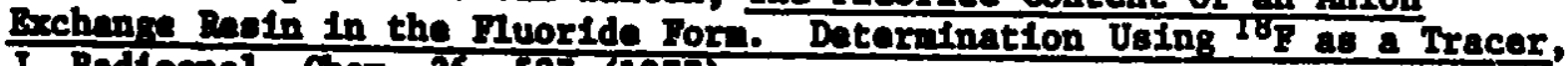
J. Radtormat. Chen. 36,587 (1977). 
87. C. De W1spelaere, Determination of Trace Amounts In Pure Iron by Neutron Activation, Thesis, Ghent State Univ. (1973), (Dutch); Nucl. Sc1. Abstr. 29, 18036 (1974).

88. C. De Wispelaere, J. P. Op De Beeck, and J. Hoste, Determination of Fourteen Elements in Pure Iron by Destructive Neutron Activation Analysib, Anal. Chim. Acta 70, 1 (1974); Anal. Abstr. 28, 1B155 (1975); Nuc1. Sc1. Abstr. 30, 2455 (1974).

89. R. M. Diamond and D. C. Whitney, Resin Selectlvity in Dilute to Concentrated Aqueous Solutions, Ion Exchange. A Series of Advances,

J. A. MarInsky, Ed., Marcel Dekker, p. 277-351 (1966).

90. E. J. Dixon and J. B. Headridge, The Anton-Exchange Separation of Titanium, Zircontum, Nloblum, Tantalum, Molybdenum and Tungsten, with Particular Reference to the Analys1s of Alloys, Analyst 89, 185 (1964); Chem. Abstr. 60, 15112 (1964); Nuc1. Sct. Abstr. 18, 17941 (1964).

91. H. -G. Döge and H. Grosse-Ruyken, Separation and Determination of Impur1t1es in Molybdenum and Tungsten by Activation AnaIysis, Isotopenpraxis 4, 262 (1968), (German); Anal. Abstr. 17, 3429 (1969).

92. G. Dominguez and J. Adrada, Radioanalytical Purification of Protactinium by Anton Exchange in Hydrochlorlc Acld-Hydrofluorlc Acld Medium, An. Quim. 67, 591 (1971), (Span1sh); Nuc1. Sc1. Abstr. 26, 52903 (1972); Anal. Abstr. 22, 2180 (1972).

93. F. Dugain, C. Castre, and B. Beyssier, Analysis of Chromium by GammeSpectrometry after Separation of the Radioelements by Ion Exchange, Ana1. Ch1m. Acta 42, 39 (1968), (French); Chem. Abstr. 69, 15806 (1968).

94. F. Dugain and J. Laverlochere, Determination of Micro Amounts of Tantalum in Niobium by Using Neutron Activation and Gama Spectrometry, Anal. Chem. 37, 998 (1965).

95. M. C. Dupuls and M. Dupuls, Separation and Purification of Ag-111, Mo-99, Te-132 and Cd-115 in a Mixture of F1ssion Products, Radioch1m. Acta 2, 4 (1963), (French); Nucl. Sc1. Abstr. 18, 5427 (1964).

96. D. N. Edgington, The Estimation of Thorium and Uranium at the Submicrogram Level in Bone by Neutron Activation, Int. J. App1. Radiat. Isot. 18, 11 (1967); Nucl. Sc1. Abstr. 21, 10271 (1967).

97. D. N. Edgington and H. F. Lucas, Jr., A System for the Neutron Activation Analysis of Trace Elements in Samples of Blological and Environmental Interest, J. Radfoanal. Chem. 5, 233 (1970); Argonne Nat1. Lab. Rev. 5, 98 (1969); Nucl. Sc1. Abstr. 24, 11485 (1970).

98. A. M. Bgorov, T. S. Kas, and Yu. I. Mitrofanov, Sorpt1on of Germantum by EDE-10P Resin In Different Ionic Forms, Rus8. J. Phys. Chem. 51, 556 (1977); [Trans1. of 2h. Flz. KhIm. 51, 944 (1977)].

99. M. M. E1-Dessouky, Anton-Exchange Separation of Thorfum and Protactinfum from Uranium Matrix, Mikrochim. Acta, 461 (1976); Anal. Abstr. 32, 6B63 (1977). 
100. A. Elek and E. Szabo, Investigation of Tungsten by Neutron Activation Analysis. II. Determination of the Sodium and Potassium Content, Acta Ch1m. (Budapest) 57, 385 (1968), (Russian); Chem. Abstr. 70, $4383 \%$ (1969).

101. H. K. E1-She.my, A. A. Abde1-Rassoul, and T. 2. B1shay, Determination of Certain Trace Impurities in Pure Lead and Aluminum by Activation Analysis, Third U. N. Conf. on Peaceful Uses of Atomfc Energy, A/Conf.28/P/829 (1964); Extract in Isot. Radiat. Technol, 2, 348 (1965); Nucl. Sci. Abstr. 18, 37071 (1964).

102. D. I. Eristav1, F. I. Brouchek, and V. D. Eristav1, Anion Exchangers In the Analytical Chemistry of Beryllium. I. and II. Tr. Gruz. Polltekh. Inst., 104(6), 23,33 (1965), (Russian); Chem Abstr. 66, 72079,72080 (1967).

103. D. I. Eristavi, F. I. Brouchek, and V. D. Eristavi, Use of Anionites in Analytical Chemistry of Beryllium. III. Tr. Gruz. Polltekh. Inst. No. 3 108, 29 (1966), (Russian); Anal. Abstr. 14, 6681 (1967); Chela. Abstr. 68, 9017 (1968).

104. D. I. Eristavf, F. I. Brouchek, and V. D. Eristav1, Anion Exchangers in the Analyt1cal Chemistry of Beryllium, Tr. Gruz. Politekh. Inst. 3(108), 41 (1966), (Russtan); Chem. Abstr. 68, 6057 (1968); Anal. Abscr. 14, 7169 (1967).

105. D. I. Eristavi, F. I. Brouchek, and V. D. Eristavi, Determination of Beryllium in Natural Water, Bul. Inst. Politeh. Ias1 13, 201 (1967), (Russian); Anal. Abstr. 15, 6330 (1968).

106. D. I. Eristav1, F. I. Brouchek, V. D. Eristav1, L. A. Berishvili, A. G. Kakabadze, and N. A. Kutslava, The Sorption of Uranyl Ions on Anton-Exchange Resins Saturated with Anlonic LIgands, Russ. J. Phys. Chem. 46, 672 (1972); [Trans1. of Zh. F1z. Khim. 46, 1165 (1972)]; Nucl. Sc1. Abstr. 27, 19550 (1973).

107. D. I. Bristav1, F. I. Brouchek, and T. G. Macharashv111, Use of AnIon Exchangers in the Analytical Chemistry of Molybdenum. I. Tr. Gruz. Politekh. Inst., No. 2, 52 (1968), (Russian); Chem. Abstr. 70, 73796 (1969).

108. D. I. Bristav1, F. I. Brouchek, and T. G. Macharashv111, Use of Antün Exchangers in the Analytical Chemistry of Molybdenum. II., Tr. Gruz. Politekh. Inst., 3(123), 53 (1968), (Russian); Chem. Abstr. 71, 45346 (1969); Nucl. Sc1. Abstr. 24, 11417 (1970); Anal. Abstr. 17, 2090 (1969).

109. D. I. Erlstav1, F. I. Brouchek, and T. G. Macharashvil1, Use of Anton Brchangers in the Analyt Ical Chemistry of Nolybdenum. III., Tr. Gruz. PoIItekh. Inst., No. 8, 19 (1968), (Russian); Chem. Abstr. 73, 126635 (1970).

110. D. I. Erlotav1, F. I. Brouchek, and T. G. Macharashvd11, Use of Anion Exchangers In the Analytical Chentstry of Molybdenum. IV., Tr. Grux. Politekh. Inst., No. 1, 30 (1969), (Russian); Chem. Abstr. 73, 51892 (1970). 
111. D. I. Eristav1, V. D. Eristav1, F. I. Brouchek, and G. V. Tsintsadze, $X$-Ray Investigation of the Fluorlde Form of an Anton Exchanger during Beryllium Sorption, Sov. Prog. Chem. 34, 87 (1968); [Trans1. of Ukr. KhIm. Zh. 34, 858 (1968)]; Chem. Abstr. 69, 99808 (1968).

112. V. D. Eristav1, D. I. Eristavi, and F. I. Brouchek, Determination of Beryllium after Its Separation by Means of an Anion-Exchange Resin, J. Anal. Chem. USSR 23, 676 (1968); [Trans1. of Zh. Anal. Khim. 23, 782 (1968)]; Chem. Abstr. 69, 40951 (1968); Anal. Abstr. 18, 56 (1970).

113. D. A. Everest and W. J. Pople1, Ion-Exchange Studies of Solutions of Tellurates, J. Inorg. Nuc1. Chem. 6, 153 (1958); Chem. Abstr. 52, 12510 (1958).

114. M. Eychenne, P. Bayle, D. Blanc, J. Laverlochere, and J. Le Strat, Determination, by (Neutron) Activation, of Impurfties in Tungsten Application to the Electric Iamp Industry, Chim. Analyt. (Parls) 49, 355 (1967), (French); Chem. Abstr. 67, 113471 (1967); Anal. Abstr. 15, 5969 (1968); Nuc1. Sc1. Abstr. 21, 38911 (1967), 23, 9322 (1969).

115. J. J. Fardy, The Adsorption of Beryllium on Ion-Exchange Resins from Inorgan1c Systems, AAEC/E-136 (1965); Nuc1. Sc1. Abstr. 19, 46230 (1965).

116. J. P. Faris, Adsorption of the Elements from Hydrofluoric. Ac1d by Anton Exchange, Ana1. Chem. 32, 520 (1960).

117. J. P. Faris, Ion Exchange - Spectroscopic Prosedures; Trace Element Analysis, The Encyclopedia of Spectroscopy, G. L. Clark, Ed., Reinhold, New York, p. 204 (1960).

118. J. P. Faris, Applications of the Copper Spark Method for Spectrographic Analysis, Sixth Conf. on Anal. Chem. In Nuclear Reactor Technology, Gatlinburg, Tenn., TID-7655, p. 193 (1962); Nucl. Sc. Abstr. 17, 40707 (1963).

119. J. P. Faris and J. K. Brody, Adsorption of Elements from HCl-HF Mixtures, P1ttsburgh Conf. on Anal. Chem. and App1. Spectroscopy, Paper No. 34 (1961).

120. N. L. Fedorova, V. I. Kurbatova, V. L. Zolotavin, M. I. Isupova, and v. K. Zharikova, Chromatographic Separation of Vanadium, Molybdenum, Tungsten, and Niobium from Fluoride Solutions, Tr. Vses. Nauchno.-Issled. Inst. Stand. Obraztsov Spectral. Etalonov 3, 82 (1967), (Russian); Chem. Abstr. 70, 16781 (1969); Nucl. Sc1. Abstr. 23, 49698 (1969).

121. T. A. Ferraro, Separation of Vanadium, Z1rcontum, T1tantum, Molybdenum, Tungsten and Columbium by Elution Development Ion Exchange Chromatography, AYRA-TR-67-10 (1967); Nucl. Sc1. Abstr. 21, 30490 (1967); Chem. Abstr. 68, 26507 (1968).

122. T. A. Rerraro, Analygis of Mtoblum Alloys, Talanta 15, 923 (1968); AD 653768 (1967); Chem. Abstr. 68, 119162 (1968); Nucl. Sc1. Abstr. 22, 48838 (1968). 
123. T. A. Ferraro, Ion-Exchange Separation of Vanadium, Z1rconium, T1tanium, Molybdenum, Tungsten and Niobium, Talanta 16, 669 (1969); Nuc1. Sc1. Abstr. 23, 28985 (1969).

124. T. M. Florence, Determination of Trace Metals in Nlobium-Iron Alloy (Abytr.), 1965 Prog. Rept., ORNL-3889, p. 29 (1966); Nucl. Sc1. Alstr. 20, 14428, 14455 (1966).

125. W. Forsling, Anion Exchange and Complex Formation of Hafnium In Hydrochlor 1c and Hydrofluorlc Acld Mixtures, Ark. Kemi 5; 489 (1953); Chem. Abstr. 47, 9199 (1953). .

126. W. Forsilng, On the Purification of Zircontum from Hafnium by Anion Exchange in Hydrochloric and Hydrofluoric Ac1d Mixtures, Ark. Kem1 $\underline{5}$, 503 (1953); Chem. Abstr. 47, 9199 (1953).

127. A. Fourcy, Contribution to the Studies on the Mineral Content of Plant Matertal Through Radioactivation Analysis, CEA-R 3386, p. 60 (1968), (French); Chem. Abstr. 70, 34837 (1969).

128. R. Frache and A. Dadone, Analys1s of Mixtures of Inorgantc Ions by Ion Exchange. V. Scheme of Quant1tative Separation of E1ghteen Cationic Species, Chromatographia 5, 581 (1972); Anal. Abstr. 24, 2680 (1973).

129. H. Freund and F. J. Miner, Determination of Aluminum in Zirconium: Based on Separation by Ion Exchange, Anal. Chem. 25, 564 (1953).

130. G. Friese and I. Wieczorek, Determination of Volume Distribution Coefficlents between Wofatits and Hydrofluoric Acld Metal Solutions, Silikattechnik 21, 175 (1970), (German); Chem. Abstr. 74, 16016 (1971).

131. W. Funasaka, M. Kawane, T. Roj1ma, and Y. Matsuda, The Separation of Fluoride Ions by Means of Ion Exchange Resing, DEG-Inf. Ser.-16, [Eng1. Trans1. of Bunsek1 Kagaku (Japan Analyst) 4, 514 (1955)]; Nuc1. Sc1. Abstr. 15, 14358 (1961).

132. E. V. Gangadharam and P. P. Parekh, Neutron Activation Analygis of Mcrogram and Submicrogram Quantitles of Thorium In Vitramafic Rocks, Radiochim. Acta 10, 65 (1968); Nuc1. Sc1. Abstr. 23, 9318 (1969); Chem. Abstr. 71, 9405 (1969).

133. M. Gastor, J. Mikulsk1, and I. Stronsk1, Radiochemical Separation of U-Th, Th-Rare Earth Elements, and Th-Pa-U on Small Ion-Exchange Columas, Nukleonlka 6, 757 (1961); Chem. Abstr. 56, 15106 (1962); ARC-Tr-5333, p. 811 (1967): Nuc1. Sc1. Abstr. 16, $8 \overline{818}$ (1962).

134. P. de Cells, Propertles and Use of Ion-Brchange Resine. II. Separation of Stcel Constituents by Ion-Brchange Besins. Anelytical Applications, Cent. Doc. 81der., Circ. Inf. Tech. 26(5), 1303 (1969), (French); Chem. Abstx. 71, 56251 (1969).

135. P. de Gelis, 8eparation by Ion Exchange Raging Applied to steel Analyale, Chen. Anal. 53, 673 (1971), (French); Anal. Abetr. 23, 270 (1972). 
136. F. Girardi and R. Pletra, Neutron Activation Analysis of Aluminum. Determination of Gamma-Emitting Impurities with Long Half Lives, Anal. Chem. 35, 173 (1963).

137. F. G1rard1, R. Pietra, and E. Sabbiont, Radiochemical Separations by Retention on Iontc Preclpitates. Adsorption Tests on 17 Materlals, Euratom Tech. Rept. EUR 4287 e, (1969); Nucl. Sc1. Abstr. 23, 47402 (1969); Chem. Abstr. 2, 73549 (1972).

138. F. Girard1, R. Pletra, and E. Sabbion1, Radiochemlcal Separations by Retention on Ionic Precipitates. Adsorption Tests on 11 Mater 1als, J. Radioanal. Chem. $\underline{5}, 141$ (1970), Chem. Abstr. $\underline{73}, 61720$ (1970).

139. 0. S. Glaso, Determfnation of Fluorine In Iron Ore and Apatite, Anal. Chim. Acta 28, 543 (1963); Chem. Abstr. 59, 4539 (1963).

140. M. M. Godneva and D. L. Motov, Separation of Fluorine from Z1rconium and Hafnlum Hydroxofluorides, J. Appl. Chem. USSR 46, 1537 (1973); [Trans1. of Zh. Prik1. KhIm. 46, 1438 (1973)]; Nucl. Sc1. Abstr. 29, 12534 (1974).

141. N. W. Golchert and J. Sedlet, Radiochemical Determination of Plutonium in Environmental Water Samples, Radiochem. Radioanal. Lett. 12, 215 (1972); Anal. Abstr. 25, 1293 (1973).

142. G. Goldstein, Equilibrium Distribution of Metal-Fluoride Complexes, Anal. Chem. 36, 243 (1964); ORNL-3620 (1964); Nucl. Sc1. Ab8tr. 19, 4104 (1965).

143. D. Gottfried, L. P. Greenland, and E. Y. Campbell, Varlation of Nb-Ta, Zr-Hf, Th-U and K-Cs in Two Diabase-Granophyre Sultes, GeochIm. Cosmochim. Acta 32, 925 (1968); Nuc1. Sc1. Abstr. 22, 48959 (1968).

144. L. P. Greenland, Simultaneous Determination of Tantalum and Hafnium in S1licates by Neutron Activation Analys18, Anal. Chim. Acta 42, 365 (1968); Nucl. Sc1. Abstr. 22, 44985 (1968).

145. L. P. Greenland and E. Y. Campbell, Determination of Nloblum In Rocks by an Isotope Dflution - Spectrophotometric Method, Anal. Chim. Acta 49, 109 (1970); Nucl. Sc1. Abstr. 24, 7413 (1970); Ane1. Abstr. 20, 972 (1971).

146. R. J. Greer, C. B. Pletr1, J. R. Weles, and A. W. Wenzel, The Bffect of Ion-Exchange Separation Procedures on Controlled-Potent1al Coulometric Analyelo, NBL-277 (1976); Nucl. Sc1. Abstr. 33, 28337 (1976).

147. H. J. Groh and C. S. Schlea, The Recovery of Neptunium-237 and Plutonium238, Progress in Nuclear Energy, Serles III, Process Chemfetry, Vo1. 4, C. B. Stevenson, B. A. Mason, and A. T. Gresky, Bds., Pergamon Press, p. 507 (1970).

148. T. N. Gudushaur1 and F. I. Brouchek, Chrometorraphle Separation of Ifloblum from Tttantum, Tungaten, Molybdenum, and Vanadium on the Fluoroform of the AB-16 Anion Exchanger, Soobohch. Akrd. Nauk Grue. SSR 70, 609 (1972), (livesian); Nucl. Sc1. Abstr. 28, 15256 (1973); Anal. Abstr. 28, 3B103 (1975). 
149. T8. N. Gudushaurf and F. I. Brouchek, Chromatographic Separation of Nloblum from Irou on the Fluortde Form of AV-16 Anionite, Soobshch. Akad. Nauk Gruz. SSR, Ser. KhIm. 1, 34 (1975), (Russian); Anal. Abstr. 31, 4B153 (1976); Chem. Abstr. 83, 157465 (1975).

150. P. Gul11ot, Preliminary Study of the Preparation of Urantum-232 by Irradiation of Protactinlum-231, CEA-R 2917 (1965), (French); Nuc1. Sci. Abstr. 20, 18802 (1966).

151. I. Hadzistelios and A. P. Grimanis, Simultaneous Determination of Arsentc, Antimony, and Mercury In Blological Materlals by Neutron Activation Analys18, Modern Trends in Activation Analysis, J. R. DeVoe and P. D. LaFleur, Bds., Natl. Bur. Stand. (U.S.) Spec. Publ. 312, Vol. I., p. 184 (1969); Chem. Abstr. 71, 12045 (1969).

152. J. L. Hague, E. D. Brown, and H. A. Bright, Separation of Titanium, Tungsten, Molybdenum, and Nloblum by Anton Exchange, J. Res. Nat1. Bur. Stand. 53, 261 (1954); Nucl. Sc1. Abstr. 9, 912 (1955).

153. J. L. Hague and L. A. Machlan, Determination of Titanium, Zirconium, and Tantalum in Steels: Separations by Anion-Exchange, J. Res. Nat1. Bur. Stand. 62, 11 (1959); Nuc1. Sc1. Abstr. 13, 7529 (1959).

154. J. I. Hague and L. A. Machlan, Determination of Mlobium and Tantalum in T1tanlum-Base Alloys, J. Res. Nat1. Bur. Stand. 62, 53 (1959); Nuc1. Sci. Abstr. 13, 9766 (1959).

155. H. Hamaguch1, K. Ish1da, and K. Ruroda, AnIon-Exchange Behavior of Rare Earths, Thorium, Protactinfum and Uranium In Thiocyanate - Chloride Media, Anal. Ch1m. Acta 33, 91 (1965); Nuc1. Sc1. Abstr. 19, 36548 (1965).

156. C. J. Hardy and C. E. Lyon, Rapld Methods for Determining the Relative Amounts of Zirconium-95 and N1oblum-95 Based on Anton-Exchange Paper Chromatography and Gamma-Spectrometry, AERE-R 4814 (1964); Nucl. Sc1. Abstr. 19, 9054 (1965).

157. C. J. Hardy, D. Scargill, and J. M. Fletcher, Studies on Protactinium(V) In Nitric Acld Solutions, J. Inorg. Nucl. Chem. 7,257 (1958); Nucl. Sc1. Abstr. 13, 2806 (1959).

158. H. Bashitan1 and T. Adach1, Spectrophotometric Determination of Traces of Nioblum in Tantalum with Chlorsulphophenol S after Separation by AnIon Bxchange, Bunsek1 Kagaku (Japan Analy8t) 24, 303 (1975), (Japanese); Anal. Abstr. 30, 1 B109 (1976).

159. J. B. Headridge, Applications of Bydrofluoric Acld and Hluorides in Analytical Chemistry, CRC Crit. Rov. Anal. Chem. 2(4), 461 (1972); Nuc1. Se1. Abetr. 20, 25177 (1972).

160. J. B. Hexdridge and B. J. Discon, The Analyels of Complex Allore, with Particular Raference to Nloblum, Tantalum and Tumaten. The Diotrubution

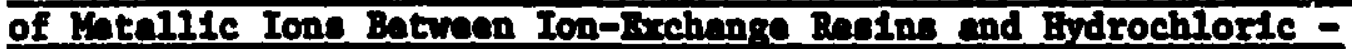
Bydroflucric Acid Solutions, Analyet 87, 32 (1962); Nucl, 8c1. Abatr. 16, $7503(1962)$. 
161. C. Heltner-Wirguin and M. Gantz, Uranyl Halide Specles Sorbed on Ion Exchanger8, Isr. J. Chem. 12, 723 (1974); Nuc1. Sc1. Abstr. 31, 13789 (1975).

162. J. 0. Hibbtts, S. Kallman, W. Giustett1, and H. K. Oberthin, A Specific Method for the Determination of Trace Amounts of Titanium, Talanta 11, 1464 (1964); Chem. Abstr. 61, 12628 (1964).

163. J. O. Hibbits, H. OberthIn, R. LIu, and S. Kallmann, The Analysis of Beryllium Oxide. VI. The Determination of Tantalum and Nlobium, Talanta 8,209 (1961); Nuc1. Sc1. Abstr. 15, 15582 (1961).

164. J. 0. Hibbits, A. F. Rosenberg, R. I. Williams, and S. Kallman, Simultaneous Determination of Trace Amounts of Zirconium and Titanium, Talanta 11, 1509 (1964); Nucl. Sc1. Abstr. 19, 4087 (1965).

165. L. E. Hibbs and D. H. Wilkins, The Determination of Aluminum, Titanium and Nickel in Their Alloys, Talanta 2, 16 (1959); Chem. Abstr. 53, 12937 (1959).

166. M. W. III1, Nuclear Decay Studies of Protactinium Isotopes, Thesis, UCRL-8432 (1958); Nucl. Sc1. Abstr. 13, 1663 (1959).

167. T. Hirao, M. Kotaka, and H. Kakihana, Separation of Boron Isotopes Us1ng a Strong Base Bxchange Resin in the Fluoride Form, Nippon Kagaku Ka1shi (8), 1477 (1973), (Japanese); Nuc1. Sc1. Abstr. 28, 29661 (1973); Ana1. Abstr. 27, 1255 (1974).

168. J. H. Holloway and F. Nelson, Ion Exchange Procedures II. Separation of Zirconfum, Neptunium and Niobium, J. Chromatogx. 14, 255 (1964); Nucl. Sci. Abstr. 18, 25294 (1964).

169. R. A. Horne, R. H. Holm, and M. D. Meyers, The Adsorption of Zinc (II) on Anlon-Exchange Resins. III. The Adsorption from Bromide, Fluoride, Cyan1de, Oxalate, Acetate, Nitrate, Phoophate, Sulfate, and Alkaline Med1a, J. Phys. Chem. 61, 1661 (1957); Nucl. Sc1. Abstr. 11, 3615 (1957).

170. J. Hoste, Determination of Trace Impurities in Semiconductors by Neutron Act Ivation Analys18, J. Radloanal. Chem. 19, 7 (1974), (French); (Proc. Int. Colloq. on Activation Anal. of Very Low Amounts of Elements, Saclay, France, 1972); Nuc1. Sc1. Abstr. 30, 2501 (1974).

171. B. A. Huff, Anton Exchange Study of a Number of Elementa in NitricBydrofluoric Acld MExtures. Analytical Applications of the System, Ana1. Chem. 36, 1921 (1964).

172. B. A. Huff, and S. J. Rulpa, Trace Impurity Analysts of Plutonium-UrantumZircontum Allors by Anton Exchange - Partition Chromatography, Anal. Chem. 38, 939 (1966).

173. B. H. Buffanan, G. M. IddInge, and R. C. HIly, Anion Exchange of Zircontum, Bafnium, Kloblum and Tantalum In Bydrochlortc Acid 8olutions, J. Am. Chem. Soc. 73, 4474 (1951). 
174. E. H. Huffman and R. C. L111y, The Anton-Exchange Separation of Zircontum and Hafinium, J. Am. Chem. Soc. 71, 4147 (1949).

175. E. H. Huffman and R. C. Lilly, Anton Exchange of Complex Ions of Hafntum and Zirconium In HCl-HF Mixtures, J. Am. Chem. Soc. 73, 2902 (1951).

176. Ionics, Inc., Technical Report for Third Quarter, 1949, NY00-1450, NYO01451 (1949); Nuc1. Sc1. Abstr. 11, 2353, 2354 (1957).

177. R. E. Isaacson and B. F. Judson, Neptunium Recovery and Purification at Hanford, Ind. Eng. Chem. Process Des. Dev. 3, 296 (1964); Chem. Abstr. 61, 12901 (1964); HW-SA-3283 (1963); Nuc1. Sc1. Abstr. 18, 13913 (1964).

178. M. M. Ivanova, I. P. Ogloblica, S. A. Cenel', V. V. Mitina, A. I. Kalinin, and V. G. Lambrev, Group Radiochemical Separation of Microimpurities in the Neutron-Activation Analysis of Special-Purity Sillca, J. Anal. Chen. USSR 32, 839 (1977); [Trans1. of Zh. Anal. Kh1m. 32, 1066 (1977)].

179. N. Jackson, F. J. G. Rogers, and J. F. Short, Final Separation and Purification of Gram Quantities of ProtactInium, AERE-R 3311 (1960); Nuc1. Sc1. Abstr. 15, 14294 (1961).

180. I. L. Jenk1ns, D. Scargill, and A. G. Wain, The Recovery and Purification of Protactinlum 231 by Anlon Exchange, J. Inorg. Nuc1. Chem. 37, 257 (1975); Nuc1. Sc1. Abstr. 31, 29180 (1975).

181. I. L. Jenkins, C. G. G. Shorthill, R. J. W. Streeton, and A. G. WaIn, The Separation of ${ }^{238} \mathrm{Pu}$ from Irradiated $237 \mathrm{~Np}$ and from F1ssion Products. Part 1 Preliminary Work. AERE-R 5074 (1966).

182. R. E. Jerv18, Present Status of Activation-Analysis Applications in Criminalistics, Isot. Radiat. Technol. 6, 57 (1968); Revised Reprint of Rad1olsotopes (Tokyo), 17, 18 (1968); Nucl. Sc1. Abstr. 22, 16453, 48835 (1968).

183. R. E. Jervis and K. Y. Wong, Chromatographic Group Separation Scheme Used with Gamma Spectrometry for Mult1-Blement Act1vation Anslys is Surveys, Proc. Symp. on Nuclear Activation Techniques in the Life Sciences, Amsterdam, May 1967, STI/PUB/155, IAEA, Vienna, p. 137 (1967); Nucl. Sc1. Abstr. 23, 4543 (1969); Chem. Abstr. 68, 75668 (1968).

184. L. U. Josht and A. K. Ganguly, Determination of ${ }^{231} \mathrm{~Pa}$ in Cosstal Sediments and Sea Vater and Disequilibrium Studies in $231 \mathrm{~Pa} / 235 \mathrm{U}$ Activity Rat 108 , J. Radloanal. Chem. 41, 15 (1977).

185. A. I. Kalinin and N. E. Kalinina, Chromatographic Separation and Complexometric Determination of Aluminum, Titantum, and Iron during Microanalysis of S1llcates, J. Anal. Chem. USSR 26, 777 (1971); [Trans1. of Zh. Anal. KhIm. 26, 881 (1971)]; Nucl. Sc1. Abstr. 25, 37294 (1971); Anal. Abstr. 23. $23 \overline{95}$ (1972). 
186. A. I. Kalinin and R. A. Kuznetsov, Radioactivation Method for the Determination of Impurities in Silicon and Sillcon Dioxide with the Use of Chromatography. Group Separation of Micro-Impurities Followed by Gamma-Spectrometric Determination, Analysis of High-Purity Materials, I. P. Alimarin, Ed. p. 99 (1968); (Transl. of 1965 Russian Publ. by Israel Prog. Sc1. Trans.)

187. A. I. Kalinin, R. A. Kuznetsov, and V. V. Molseev, Activation Analysis of Silicon Dioxide, Sov. Radiochem. 4, 505 (i962); [Trans1. of Radiokhim. 4, 575 (1962)]; Nucl. Sc1. Abstr. 17, 19979 (1963).

188. A. I. Kalinin, R. A. Kuznetsov, and V. V. Molseev, Radioactivation Analysis of Silicon Dioxide by Means of Ion Exchange Chromatography. Part 4. Separation of Elements on an Anfon Exchanger from Solution of Hydrofluoric AcId and a Mxture of Hydrofluoric and Hydrochloric AcId, Radiochemical Methods of Determining Trace Elements, Acad. Sc1. USSR, Moscow-Leningrad, p. 176 (1965), (Russian); Nucl. Sc1. Abstr. 20, 20635 (1966); Chem. Abstr. 63, 10659 (1965).

189. A. I. Kallnin, R. A. Kuznetsov, V. V. Molseev, and A. N. Murin, The Use of Ion-Exchange Chromatography in Radloactivation Analysis of Trace Impurities in Silica, Proc. Acad. Sci. USSR, Chem. Sec. 141, 1089 (1961); [Trans1. of Dok1. Akad. Nauk SSSR 141, 98 (1961)]; Nuc1. Sc1. Abstr. 16, 6377 (1962).

190. A. I. Kalinin, R. A. Kuznetsov, V. V. Molseev, and V. E. Tsepurnek, Radioactivation Analysis of Silicon Dioxide Using Ion Exchange Chromatography. I. Group Separation of Trace Impurities Followed by GammaSpectrometric Determination, Radiochemical Methods of Determining Trace Elements, Acad. Sci. USSR, Moscow-Leningrad, p. 161 (1965), (Russian); Nucl. Sc1. Abstr. 20, 20632 (1966); AD-662383. Eng1. Trans. FTD-MT-66-142 (1967); Nuc1. Sc1. Abstr. 22, 12227 (1968); Chem. Abstr. 63, 10658 (1965).

191. A. I. Kalinin, R. A. Kuznetsov, and I. L. Osipchuk, The Ion Exchange Behavior of Arsenic in the Solutions of Hydrofluorlc Acld, Vestn. Leningrad. UnIv., Fiz. Khim. 23, (16) 155 (1968), (Russian); Chem. Abstr. 70, 14704 (1969); Anal. Abstr. 17, 3409 (1969).

192. S. Kallman, Spectrophotometric Determination of Trace Amounts of Z1rconium, Titanium, and Molybdenum In Tungsten, Anal. Chem. 36, 2358 (1964).

193. S. Kallman, N1oblum and Tantalum, Treatise on Analytical Chemistry, Part II, Vol. 6, I. M. Kolthoff and P. J. Elving, Eds., Interscience, p. 177 (1964).

194. S. Kallman and H. K. Oberthin, Separation and Determination of Rhenlum by Anton Exchange Using the Fluortde-Chlortde System, Anal. Chem. 37, 280 (1965).

195. S. Kallman, H. Oberthin, and R. LIU, Determination of N1oblum and Tantalum In Minerals, Ores, and Concentrates Using Ion Exchange, Ana1. Chem. 34. 609 (1962). 
196. D. G. Karraker, Recovery of Reactor - Produced Pa-231, Radiochim. Acta 14, 158 (1970); Nuc1. Sc1. Abstr. 25, 13100 (1971).

197. L. I. Katzin, R. G. Larson, R. C. Thompson, and Q. Van Winkle, Recovery of Protactinfum from Ores, U.S. Pat. 2,887,355 (1959); Chem. Abstr. 53, 18678 (1959).

198. C. Keller, Comparative Investigations on the Behavior of Nioblum, Tantalum and Protact Inium on Ion Exchangers, Radioch1m. Acta 1, 147 (1963), (German); Chem. Abstr. 60, 9951 (1964).

199. C. Keller, The Chemistry of Protactinium, Angew. Chem. Int. Ed. In Engl. 5, 23 (1966); Nuc1. Sc1. Abstr. 20, 18638 (1966).

200. W. KiesI, Determination of Trace Elements in Meteorites by Activation Analysis. I. Selentum, Arsentc, Antimony, Tin and Mercury (Chromium and Cobalt), Z. Anal. Chem. 227, 13 (1967), (German); Nuc1. Sc1. Abstr. 21, 21893 (1967); Chem. Abstr. 67, 17514 (1967).

201. W. Kies1, Determination of Trace Elements in Meteorites by Neutron Activation Analysis, Modern Trends In Activation Analysis, J. R. Devoe and P. D. LaFleur, Eds., Nat1. Bur. Stand. (U.S.) Spec. Publ. 312, Vol. I, p. 302 (1969).

202. J. I. KIm and H. -J. Born, Anlon Exchange Behavior of Thorium, ProtactInium, Urantum and Other Elements in Hydrochloric Acid and Acet Ic AcId Mixtures, Radlochim. Acta 14, 35 (1970); Nuc1. Sc1. Abstr. 24, 48164 (1970).

203. J. I. KIm and H. -J. Born, Anion Exchange Behaviour and Separation of Thorium, Protactinium and Uranfum In Nitric and Acetic Acid Mixtures, Radlochim. Acta 14, 65 (1970); Nuc1. Sc1. Abstr. 24, 48154 (1970).

204. J. I. KIm and H. -J. Born, The Production of ${ }^{231} \mathrm{~Pa}$ and ${ }^{232} \mathrm{U}$ by Reactor Neutron Irradiation of $230 \mathrm{Th}$ and $232 \mathrm{Th}$ Mixture, Radioch1m. Acta 16, 160 (1971); Nuc1. Sc1. Abstr. 26, 25387 (1972).

205. J. I. KIm, H. Lagally, and H. -J. Born, Study on the Anton Exchange Behavior and Separation of Protactinium, Zirconfum, and Niobium in $\mathrm{HAc}_{\mathrm{HNO}}$-HF Mixtures, J. Inorg. Nucl. Chem. 33, 3547 (1971); Nuc1. Sc1. Abstr. 26, 9045 (1972); Anal. Abstr. 23, 2289 (1972).

206. J. I. KIm, H. Lagally, and H. -J. Born, Ion Exchange In Aqueous and in Aqueous-Organic Solvents. Part I. Anfon-Exchange Behavior of $\mathrm{Zr}$, Nb, Ta and $\mathrm{Pa}$ in Aqueous HCl-HF and in HCI-HF-Organic Solvent, Ana1. Chim. Acta 64, 29 (1973); Nuc1. Sc1. Abstr. 27, 19530 (1973); Anal. Abotr. 25, 2142 (1973).

207. H. W. Kirby, The Radiochemigtry of Protactinium, NAS-NS-3016 (1959), (Avallable from the Office of Technical Services, Dept. of Commerce, Washington, D.C.).

208. M. K1rk, E. G. Perry, and J. M. Arr1tt, Separation and Atomic-Absorption Neasurement of Trace Amounts of Lead, StIver, Zinc, Blsmuth and Cadmium In High-NIckel Alloys, Anal. ChIm. Acta 80, 163 (1975); Anal. Abstr. 30, 6B218 (1976). 
209. R. Ko, A. C. Leaf, W. Y. Matsumoto, H. A. Treibs, and M. R. Weiler, Analytical Methods for Alkaline Earth Determinations in Support of the Strontlum-90 Recovery Program, HW-69677 (1961); Nuc1. Sc1. Abstr. 16, 23601 (1962).

210. K. Komura and M. Sakanoue, ConsecutIve AnIon-Exchange Separation of Radium, Thorium, Protactintum and Uranium from an Iron Carrier, Bunsek1 Kagaku (Japan Analyst) 16, 114 (1967), (Japanese); Anal. Abstr. 15, 6492 (1968); Nucl. Sc1. Abstr. 21, 15987 (1967); Chem. Abstr. 67, 86853 (1967).

211. J. Kork1sh, Analytical Chemistry of Zirconium. VI. Method for the Determination of Zirconium In Alloys, Z. Anal. Chem. 176, 403 (1960), (German); Chem. Abstr. 55, 7146 (1961).

212. J. Kork1sch, The Determination of Titanlum in Natural Water, $Z$. Anal. Chen. 178, 39 (1960), (German); Chem. Abstr. 55, 12712 (1961).

213. J. Korkisch, Method for the Spectrophotometric Determination of Titanium In Steel, Mikrochim. Acta 262 (1961), (German); Chem. Abstr. 55, 16268 (1961).

214. J. Korkisch, Ion-Exchange Separation of Uranium and Thorium from Ore Pulps and Clear 'olutions, Atomic Energy Review, Vol. III, No. 3, IAEA, Vienna, p. 535 (1970); Nucl. Sci. Abstr. 25, 2330 (1971).

215. J. Kork1sch, G. Arrhenfus, and D. P. Kharkar, Spectrophotometric Determination of Titanium after Separation by Anion Exchange, Anal. Chim. Acta 28, 270 (1963); Chem. Abstr. 58, 13131 (1963).

216. J. Korkisch and A. Farag, Analytical Chemistry of Zirconium, III. A New Method for the Concentration of Zirconium with Strongly Basic Anion Exchangers: Application to the Determination of Z1rconium in S1licate Rocks, Z. Anal. Chem. 166, 170 (1959), (German); Chem. Abstr. 53, 16825 $\overline{(1959) .}$

217. K. A. Kraus and G. E. Moore, Separation of Zirconium and Hafnium with Anton Exchange Resins, J. Am. Chem. Soc. 71, 3263 (1949).

218. K. A. Kraus and G. E. Moore, Separation of Columblum and Tantalum with Anton Exchange Resins, J. Am. Chem. Soc. 71, 3855 (1949).

219. K. A. Kraus and G. E. Moore, Anton Exchange Studies. I. Separation of Zirconium and Niobium In HCI-HF Mxtures, J. Am. Chem. Soc. 73, 9 (1951); ORNL-661 (1950); Nucl. Sc1. Abstr. 4, 4241 (1950).

220. K. A. Kraus and G. E. Moore, Anfon Exchange Studies. II. Tantalum In Some HF-HC1 Mixtures, J. Am. Chem. Soc. 73, 1.3 (1951); ORNL-662 (1950); Nuc1. Sc1. Abstr. 4, 3713 (1950).

221. K. A. Kraus and G. E. Moore, Anfon Exchange Stud1es. III. Protactinfum In Some HCI-HF Mixtures: Separation of Niobium, Tantalum and Protactinium, J. Am. Chem. Soc. 73, 2900 (1951); 0RNL-673 (1950); Nucl. Sc1. Abstr. $\underline{5}$ 990 (1951). 
222. K. A. Kraus and G. E. Moore, Anton-Exchange Studies. XV. Separation of Protactinfum and Iron by Anion-Exchange in HCl-HF Solutions, J. Am. Chem. Soc. 77,1383 (1955).

223. K. A. Kraus, G. E. Moore, and F. Nelson, Anlon-Exchange Studies. XXI. Th(IV) and U(IV) in Hydrochloric Actd. Separation of Thorium, Protactinium and Uranium, J. Am. Chem. Soc. 78, 2692 (1956).

224. K. A. Kraus and F. Nelson, Anlon Exchange Studles of the Flssion Products, Proc. Int. Conf. on Peaceful Uses Atomic Energy, Geneva, 1955, 7, 113 (1956).

225. K. A. Kraus and F. Nelson, Metal Separations by Anton Exchange, Symposium on Ion Exchange and Chromatography In Analytical Chemistry, ASTM Spec. Tech. Pub. No. 195, p. 27 (1956).

226. K. A. Kraus, F. Nelson, and G. E. Moore, Anion-Exchange Studies. XVII. Molybdenum(VI), Tungsten(VI) and Uranium(VI) In HCI and HC1-HF Solutions, J. Am. Chem. Soc. 77, 3972 (1955).

227. K. A. Kraus, H. O. PhIllips, and F. Nelson, Some Applications of Radio1sotopes in Physical Chemistry--Two-Phase Equilibria and Packed-Colum Techniques, Proc. 1960 Copenhagen Conf., Radiolsotopes in the Physical Sclences and Industry, IAEA, VIenna, Vol. 2, p. 387 (1962).

228. W. J. Rrause, Method of Separating F1ssion Product Elements, Actinldes, and Aluminum by Means of Ion Exchange, KFK-2192 (1975), (German); Nuc1. Sc1. Abstr. 33, 6929 (1976); Ana1. Abstr. 31, 4B91 (1976).

229. I. K. Kressin and G. R. Waterbury, The Quantitative Separation of Plutonium from Various Ions by Anion Exchange, Anal. Chem. 34, 1598 (1962).

230. H. L. Krieger, J. E. Gilchrist, and S. Gold, Concentration of Radioactivity and Detection of Cobalt -60 and Zinc-65 in Ralnout, Talanta 6, 254 (1960); Chem. Abstr. 55, 6172 (1961).

231. M. Krivanek, F. Kukula, and V. V1ns, Determination of Some Impurtt1es In Gallium by Neutron Activation, J. Radioanal. Chem. 1, 219 (1968), (French); Nucl. Sc1. Abstr. 22, 42614 (1968).

232. J. Krt11, A. Moravec, and J. Mencl, Isolation of Zirconlum Isotopes from Flssion Products for Mass Spectrometric Determination, Radiochem. Radioanal. Lett. 10, 83 (1972); Nucl. Sc1. Abstr. 26, 35803 (1972); Anal. Abstr. 23, 4555 (1972).

233. S. M. Ku, Emisgion-Spectrographic Analysis for Trace Elements In Iron and Steel, Chemlstry, Talpel (3), 85 (1971), (Chinese); Anal. Abstr. 24, 3469 (1973).

234. V. I. Kurbatova, G. N. Emasheva, and N. D. Redorova, Chromatograph1c Separation of Yolybdenum, Tungsten, and Nioblum in Fluoride Media, Tr. Vees. Nauchno.-Issled. Inst. Stand, Obraztsov Spektr. Etalonov 7, 102 (1971), (Russtan); Nuc1. Sc1. Abstr. 28, 2193 (1973); Anal. Abstr. 24, 125 (1973). 
235. F. J. Langmyhr and S. Sveen, Decomposability in Hydrofluoric Acld of the Main and Some Minor and Trace Minerals of S1llcate Rocks, Anal. Chim. Acta 32, 1 (1965); Chem. Abstr. 62, 3642 (1965).

236. A. K. Lavrukhina and Z. K. Mil'nikova, Anion-Exchange Method for Separating Mixtures of Elements Composing Meteoritic Material, J. Anal. Chem. USSR 24, 1390 (1969); [Trans1. of Zh. Anal. KhIm. 24, 1714 (1969)]; Chem. Abstr. $\underline{72}, 62425$ (1970).

237. A. K. Lavrukhina and N. K. Sazhina, Separation of Iron, Nickel, and Cobalt in Acetone-Mineral Acid Media on Strongly Basic Anion-Exchange Resins, J. Anal. Chem. USSR 24, 694 (1969); [Trans1. of Zh. Anal. KhIm. 24, 870 (1969)]; Chem. Abstr. 71, 56390 (1969).

238. A. C. Leaf, The Determination of Z1rconium-95 Plus N1obium-95 by AnionExchange Separation from Other Fission Products, Talanta 6, 265 (1960); HW-62188 (1959); Ana1. Abstr. 7, 4722 (1960).

239. I. A. Lebedev, S. V. Pirozhkov, V. M. Senochkin, and G. N. Yakovlev, Ion-Exchange Isolation of Protactinium, and Properties of Some ProtactInfum Compounds, Sov. Radiochem. 3, (6), 258 (1961); [Trans1. of Rad1okhim. 3, 760 (1961)]; Nuc1. Sc1. Abstr. 16, 11662 (1962).

240. G. Leliaert, Determination of Trace Element Diffusion In Quartz and In Germantum, Pure App1. Chem. 1, 121 (1960); Nuc1. Sci. Abstr. 15, 8727 (1961).

241. A. Lesbats and M. R. Tardy, Activation Analysis at the Saint-Etienne School of Mines: Application to the Study of Very-High-Purity Iron, J. Radioanal. Chem. 17, 127 (1973), (French); (Proc. Int. Colloq. on Activation Analysis of Very Low Amounts of Elements, Saclay, France, 1972); Nuc1. Sc1. Abstr. 28, 2122 (1973); 29, 28979 (1974).

242. R. Lteberman and A. A. Mogh1ssi, Coprecipltation Technique for Alpha Spectroscopic Determination of Uranium, Thorfum, and P1utonium, Health Phys. 15, 359 (1968); Nuc1. Sc1. Abstr. 22, 48839 (1968).

243. J. P. Lopez and G. Dominguez, Radioanalytical Separation of Molybdenum-99 In F1ssion Products, An. Quim. 66, 669 (1970), (Spantsh): Anal. Abstr. 21, 115 (1971).

244. W. Maenhaut, Neutron-Activation Analysis of U1tra-Pure TIn, Verh. K. Acad. Wet., Lett. Schone Kunsten Belg., K1. Wet. $37(134), 109$ (1975), (Dutch); Anal. Abstr. 30, 5B124 (1976); Chem. Abstr. 83, 157465 (1975).

245. W. Maenhaut, F. Adams, and J. Hoste, Determination of Trace Impurities In TIn by Neutron Activation Analysis. II. Determination sf Indium and Manganese, J. Radioanal. Chem. 9, 27 (1971); Nuc1. Sc1. Abstr. 26, 17352 (1972); Anal. Abstr. 23, 197 (1972).

246. W. Maenhaut, F. Adams, and J. Hoste, Neutron Activation Analygis of 쁠-Purity Tin. Chemical Separations and Nuclear Interferences, Anal. ChIm. Acta. 59, 209 (1972); Nuc1. Sc1. Abstr. 26, 35767 (1972). 
247. E. A. Materova and T. I. Rozhanskaya, Potentiometric and Ion-Exchange Investigation of Boron in Fluoroboric Solutions, Russ. J. Inorg. Chem. 6, 87 (1961); [Trans1. of Zh. Neorg. KhIm. 6, 177 (1961)]; Chem. Abstr. 56, $9693(1962)$.

248. K. C. McBride, Ion-Exchange Separation and Mass-Spectrometric Analysis of Uranium for Solutions Containing Plutonium, U.S. ERDA Rept. ARH-SA-219 (1975); Ana1. Abstr. 31, 3B79 (1976).

249. J. J. McCormack, F. H. Cripps, and W. A. Wiblin, Determination of Protactinium-233 in Irradiated Thortum, Anal. Chim. Acta 22, 408 (1960); Nuc1. Sc1. Abstr. 14, 24083 (1960).

250. J. P. McKaveney, Evaluation of Some Analyt1cal Procedures for N1oblumBase Alloys, Anal. Chem. 35, 2139 (1963).

251. H. R. McLendon, Sol1 Monitoring for Plutonium at the Savannah River Plant, Health Phys. 28, 347 (1975); Nucl. Sci. Abstr. 32, 3493 (1975).

252. J. W. McMillan, J. W. Haynes, R. J. Bentham, and J. D. Cunningham, The Determination of T1tanium-44 in Bremsstrahlung-Irradiated Titanium Fo11, AERE-R 6203 (1969); Anal. Abstr. 20, 3820 (1970); Chem. Abstr. 72, 62493 (1970).

253. C. E. Mellish, The Separation of $\mathrm{Mn}-54, \mathrm{Co}-56, \mathrm{Co}-58, \mathrm{Zn}-65$, and $\mathrm{Nb}-95$ In a Carrier-Free State by the Use of Anion Exchange Resin, AERE-1/M-39 (1955); Nuc1. Sc1. Abstr. 10, 6590 (1956); Chem. Abstr. 50, 9169 (1956); (In Ref. 362, p. 48).

254. E. P. Mignonsin, Analysis of Selenium by Neutron Radioactivation. Application of Ion Exchange Chromatography on Resins to the Systematic Analysis of Selentum, J. Radioanal. Chem. 19, 33 (1974), (French);

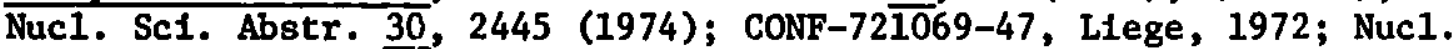
Sc1. Abstr. 27, $22 \overline{065}$ (1973).

255. E. P. Mignonsin, Analysis of Blological Samples by Radioactivation: Application of Ion Exchange Chromatography in the Systemat1c Analysis of Samples of Fish, Radiochim. Acta 22, 183 (1975), (French).

256. E. P. Mignonsin and P. Albert, Analysis of Pure Zircon by Neutron Radioactivation, Bull. Soc. Chim. Fr. (2) 553 (1965), (French); Chem. Abstr. 62, 15406 (1965); Nucl. Sc1. Abstr. 19, 24418 (1965).

257. E. P. Mignonsin and I. Roelandts, Application of Radiochemical NeutronActivation Analysis to the Determination of Selenium and Teliurium in Geological Mater1als, Chem. Geol. 16, 137 (1975); Anal. Abstr. 32, 2B144 (1977); Chem. Abstr. 84, 77152 (1976).

258. B. MInamt, M. Honda, and Y. Sasak1, Ion-Exchange Separatton of Fission Products, Bull. Chem. Soc. Jpn. 31, 372 (1958); Nucl. Sci. Abstr. 12, 13829 (1958). 
259. V. V. Molseev, R. A. Kuznetsov, and A. I. Kalinin, The Radioactivation Analysis of Silicon and Silicon Compounds with the Successive Use of Ion-Exchange Chromatography, Proc. Conf. on Modern Trends in Activation Analys1s, College Sta., Texas, 1965, p. 164, Nucl. Sc1. Abstr. 19, 46228 (1965).

260. V. V. Molseev, R. A. Kuznetsov, and A. I. Kalinin, Neutron-Act1vation Analysis of Sillca Using Ion-Ex:change Chromatography, Activation Analys1s, E. M. Lobanov, Ed., Engl. Trans1. of Proc. First Al1-Union Coord. Conf., Tashkent, October 1962, p. 36 (1964); U.S. Dept. Comm. AEC-TR-6639 (1966); ORNL-TM-1505, p. 3, (1966); Nucl. Sci. Abstr. 20, 33166 (1966).

261. G. E. Moore and K. A. Kraus, Anion Exchange Studies of Urantum(VI) in HCl-HF Mixtures. Separation of $\mathrm{Pa}(\mathrm{V})$ and $\mathrm{U}(\mathrm{VI})$, Prog. Rept. ORNL-795, p. 16 (1950); Nuc1. Sc1. Abstr. 11, 8283 (1957).

262. R. W. Moshter, Ion Exchange and Chromatography, Separations and Determinations, Analytical Chemistry of Niobium and Tantalum, Macmillan, N.Y., P. 93 (1964).

263. P. Muenchow, Determination of Nioblum and Tantalum In Stab1lized ChromeN1cke1 Stee1s, Chem.-Ztg. 88, 37 (1964); Chem. Abstr. 60, 9906 (1964).

264. A. K. Mukherj1, Separations Using Ion-Exchange, Analytical Chemistry of Zirconlum and Hafnium, Pergamon Press, Chap. 8 (1970); Nucl. Sc1. Abstr. 24, 46181 (1970).

265. B. I. Nabivanets and V. V. Lukachina, Tantalum(V) Complexes in Solution, Sov. Prog. Chem. 37, 60 (1971); [Trans1. of Ukr. Kh1m. Zh. 37, 581 (1971)]; Nucl. Scĭ. Abstr. 26, 6724 (1972).

266. T. NakanishI and M. Sakanoue, Ion-Exchange Separation of the Daughter of Protactintum-231, Radiochim. Acta 11, 119 (1969); Nuc1. Sc1. Abstr. 23, 38036 (1969).

267. H. Natsume, H. Unezawa, S. Okazak1, T. Suzuk1, T. Sonobe, and S. Usuda, Sequentlal Ion-Exchange Separation of Heavy Elements and Selected F1ssion Products for Burn-Up Measurement, J. Nucl. Sc1. Techno1. 9, 737 (1972); Anal. Abstr. 25, 3019 (1973).

268. R. Neirinckx, F. Adams, and J. Hoste, Determination of Impurities in Titanium and T1tanlum Dioxide by Neutron Activation Analysis. I. Simultane ous Determination of 16 Trace Elements in Titanium, Anal. Chim. Acta 43, 369 (1568); Chem. Abstr. 70, 8603 (1969); Anal. Abstr. 18, 2331 (1970).

269. R. Neirinckx, F. Adams, and J. Hoste, Determination of Impurities in Titantum and Titanium Dloxide by Neutron Activation Analysis. II. Determination of 27 Trace Constituents in Titania Powder, Anal. Ch1m. Acta 46, 165 (1969); Nucl. Sc1. Abstr. 23, 31318 (1969). 
270. R. Nelrinckx, F. Adams, and J. Hoste, Determination of Impurities in Titanium and Titanium Dioxide by Neutron Activation Analysis. III. Determination of Vanadium and Aluminum in Titanium and Titania by Preseparation, Anal. Chim. Acta 47, 173 (1969); Nucl. Sc1. Abstr. 25, 55 (1971).

271. R. Neirinckx, F. Adams, and J. Hoste, Determination of Impurities in TItanlum and TItanium Dioxide by Neutron Activation Analysis. IV. Determination of Trace Impurities in Titanium Dioxide Single Crystals, Anal. Ch1m. Acta 48, 1 (1969); Nuc1. Sc1. Abstr. 24, 22 (1970).

272. R. Neirinckx, F. Adams, and J. Hoste, Determination of Impurities In Titanium and Titanium Dloxide by Neutron Activation Analysis. V. Destructive and Non-Destructive Determination of Manganese, Indfum and Uranium, Anal. ChIm. Acta 50, 31 (1970); Nuc1. Sc1. Abstr. 24, 22616 (1970).

273. D. M. Ne1son, E. M. Yaguch1, B. J. Waller, and M. A. Wahlgren, Radiochemical Methods (Analysis of Ashed Bfological Samples for Plutonium), Radio1. Environ. Res. Div. Ann. Prog. Rept., Jan.-Dec. 1973, Argonne National Laboratory Report ANL-8060, (Pt. 3), (1973).

274. F. Nelson, K. A. Kraus, R. M. Rush, and D. C. Mtchelson, Chemlstry of Aqueous Systems. Anion Exchange Studies, Chem. Div. Annu. Prog. Rept. for Perlod Ending June 20, 1957, ORNL-2386, p. 92 (1957).

275. F. Nelson, D. C. Michelson, and J. H. Holloway, Ion Exchange Procedures III. Separation of Uranium, Neptunium and Plutonfum, J. Chromatogr. 14, 258 (1964); Nucl. Sc1. Abstr. 18, 25295 (1964).

276. F. Nelson, R. M. Rush, and K. A. Kraus, Anton Exchange Studies. XXVII. Adsorbibility of a Number of Elements in HCl-HF Solutions, J. Am. Chem. Soc. 82, $339(1960)$.

277. M. Neuburger and A. Fourcy, Determination of Molybdenum and Tungsten In Plants by Neutron ActIvation. Radiochemical Method Adapted to Plants RIch in Calctum, J. Radioanal. Chem. 1, 289 (1968), (French); Nuc1. Sc1. Abstr. 22, 47082 (1968).

278. S. Niese, Neutron Activation Analysis of Semiconductor Silicon, J. Radioana1. Chem. 38, 37 (1977).

279. M. K. Nikitin, Investigation of Ion Exchange in HF Solutions. Group Separation of Elements by Means of Ion-Exchange Resins, Dok1. Chem. 148, 74 (1963); [Trans1. of Dok1. Akad. Nauk SSSR. Ser. Kh1m. 148, 595 (1963)]; Nucl. Se1. Abstr. 17, 16152 (1963).

280. M. K. N1kitin and G. S. Katykhin, Study of Ion Exchange in Hydrofluoric Acld Solutions. Separation of Lead-210, Bismuth-210 and Polonium, Sov. At. Energy 14, 516 (1963); [Trans1. of At. Energ. 14, 493 (1963)]; Ana1. Abstr. 7, 2499 (1964); Nuc.1. Sc1. Abstr. 17, 34055 (1963).

281. N. S. N1kolaev and Yu. A. Buslaev, I. Solubility and Hydrolysis in the $\mathrm{HP}-\mathrm{MbF}_{5}-\mathrm{H}_{2} \mathrm{O}$ System, Russ. J. Inorg. Chem. 4, 84 (1959); [Trans1. of $\mathrm{Zh}$. Neorg. Kh1m. 4, 205 (1959)]; Chem. Abstr. 53, 11972 (1959). 
282. K. Nishimura and H. Irokawa, Spectrophotometric Method for Determination of Niobium in Tantalum and Tantalum Oxide, Bunsek1 Kagaku (Japan Analyst) 13, 304 (1964), (Japanese); Nuc1. Sc1. Abstr. 18, 25284 (1964); Chem. Abstr. 61, 1261 (1964).

283. Oak Ridge National Laboratory, Chemical Technology Division Annual Progress Reports:

a. Production of Urantum-232; Chem. Processing of Urantum, ORNL-3314, p. 162 (1962); Nuc1. Sc1. Abstr. 16, 32890 (1962).

b. Production of Urantum-232; Chem. Processing of Urantum, ORNL-3452, p. 239 (1963); Nuc1. Sc1. Abstr. 17, 39200 (1963).

c. Curlum Processing, ORNL-3452, p. 134 (1963); Nuc1. Sc1. Abstr. 17, 39182 (1963).

d. Chemistry of Protactinium, ORNL-3627, p. 216 (1964); Nuc1. Sc1. Abstr. 19, 4252 (1965).

e. Chemistry of Protactinlum, ORNL-4145, p. 260 (1!67); Nucl. Sc1. Abstr. 21, 45082 (1963).

284. R. S. Ondrejcin, Determination of Uranium Isotopes In Irradiated Thorium, DP-883 (1964); Nucl. Sc1. Abstr. 18, 33462 (1964).

285. Z. I. Otmakhova, 0. V. Chashchina, and G. A. Kataev, Use of Ion-Exchange Resins in Spectrochemical Analysis, Isv. S1b. Otd. Akad. Nauk SSSR, Ser. KhIm. Nauk (4) 87 (1967), (Russian); Chem. Abstr. 69, 15719 (1968).

286. V. S. Pakholkov, Anion Exchange in Hydrofluoric Acld Solutions and Its Application for the Separation of Elements, Abstracts, XXth. Int. Cong. Pure and App1. Chem., Moscow, USSR, 1965, CONF-650723 (Abstr.), p. 177 (1965); Nuc1. Sc1. Abstr. 20, 20552 (1966).

287. V. S. Pakholkov, The Problem of the Uranium Sorption Mechanism on AntonExchange Resins from Uranyl Fluoride Solutlons, AEC-Tr-6716, p. 183 (1967); [Trans1. of Coprecipitation and Adsorption of Radioactive Elements, V. M. Vdovenko, Ed., Moscow-LenIngrad, p. 162 (1965); Nuc1. Sc1. Abstr. 19, 32313 (1965); Chem. Abstr. 63, 6357 (1965).

288. V. S. Pakholkov, The Sorption of Uranium(VI) on the Strongly Basic AV-17 Anton-Exchange Resin from Hydrofluoric Ac1d Solutions, AEC-Tr-6716, p. 192 (1967); Ib1d., p. 169; Nucl. Sc1. Abstr. 19, 32315 (1965); Chem. Abstr. 63, 6356 (1965); CRA-Tr-R-1614 French Transi. (1966).

289. V. S. Pakholkov, The Sorption of Uranlum(VI) on Anion-Exchange Resins from $\mathrm{H}_{2} \mathrm{SO}_{4}$-HF Solutions, AEC-Tr-6716, p. 198 (1967); Ib1d., p. 174; Nucl. Sc1. Abstr. 19, 32316 (1965); Chem. Abstr. 63, 6357 (1965).

290. V. S. Pakholkov, The Sorption of Uranlum(VI) from HCl-HF Solutione on Anfon-Exchange Resins, AEC-Tr -6716 , p. 203 (1967); Ib1d., p. 178; Nuc1. Sc1. Abstr. 19, 32317 (1965); Chem. Abstr. 63, 14021 (1965). 
291. V. S. Pakholkov, Sorption of Quinquevalent Vanadium from Hydrofluoric Acid Solutions by Anion-Exchange Resins, Izv. Vyssh. Uchebn. Zaved., KhIm. Khim. Tekhnol. 8, 940 (1965), (Russian); Chem. Abstr. 64, 16676 (1968).

292. V. S. Pakholkov, Sorption of Vanadium(IV) from Hydrofluoric Actd Solutions by Anton-Exchange Resins, Izv. Vyssh. Uchebn. Zaved., KhIm. KhIm. Tekhno1. 9, 242 (1966), (Russian); Chem. Abstr. 65, 11425 (1966).

293. V. S. Pakholkov, Anton-Exchange Separation of Elements of the Chromfum Sub-Group in Fluorlne-Containing Medla, Tr. Nauchno.- Issled. Inst. Khromatograf. Voronezh. Univ. (2), 166 (1968), (Russian); Anal. Abstr. 18, 3943 (1970); Chem. Abstr. 74, 25354 (1971).

294. V. S. Pakholkov and G. V. Bazuev, Effect of Small Additions of Hydrofluoric Acid on the Sorption of Urantum from Uranyl Sulfate Solutions by Anton Exchangers, Tr. Ural. Politekh. Inst. No. 148, 75 (1966), (Russian); Chem. Abstr. 68, 99018 (1968).

295. V. S. Pakholkov, V. P. Ganvaev, and A. D. U1'yanova, Sorption of Aluminum and Gallium Subgroup Elements from Fluorine-Containing Solutions by AV-17, EDE-10P, and AN-2F Anion Exchangers, Izv. Vyssh. Uchebn. Zaved., Tsvetn. Metal1. 15, 79 (1972), (Russian); Chem. Abstr. 79, 35424 (1973).

296. V. S. Pakholkov and A. Ya. Korbut, Antonic Sorption of Vanadium from HF-HCl Solutions, Izv. Vyssh. Uchebn. Zaved., Tsvetn. Meta11. 5, 100 (1962), (Russian); Chem. Abstr. 58, 7398 (1963).

297. V. S. Pakholkov and A. Ya. Korbut, Separation of Vanadium and Uranium in Fluortde-Contalning Solutions by Means of Antonites, Izv. Vyssh. Uchebn. Zaved., Tsvetn. Metal1. 6, 116 (1963), (Russian); Anal. Abstr. 12,90 (1965); Chem. Abstr. 60, 1365 (1964); Nucl. Sc1. Abstr. 19, $\overline{15678}$ (1965).

298. V. S. P.ikholkov and I. B. Maksimov, Separation of Nioblum and Tantalum In Hydrofluoric Acid Solutions with the Ald of EDE-10P and AN-2F Anion Exchange Resins, J. Appl. Chem. USSR 39, 481 (1966); [Trans1. of Zh. Prikl. Kh1m. 39, 507 (1966)]; Chem. Abstr. 65, 11 (1966).

299. V. S. Pakholkov and I. E. Maksimov, Separation of N1ob1um and Tantalum in HCl-HF and $\mathrm{H}_{2} \mathrm{SO}_{4}$ Solutions by Strongly Basic AV-17 Anion-Exchanger, J. App1. Chem. USSR. 39, 1103 (1966); [Trans1. of Zh. Prikl. KhIm. 39, 1179 (1966) ]; Chem. Abstr. 65, 7983 (1966).

300. V. S. Pakholkov and V. D. $01^{\prime}$ kh1n, Sorption of Molybdenum from HC1-HF Solutions by AV-17, EDR-10P, and AN-2F Anion Exchange Resins, J. AppI. Chem. USSR 38, 981 (1965); [Trans1. of Zh. PrIk1. Khim. 38, 993 (1965)]; Chem. Abstr. 63, 3654 (1965).

301. V. S. Pakholkov and V. D. 01'kh1n, Separation of Tungaten and Yolybdenum in Fluorine-Contalzing. Solutions by Means of the Ion Exchangers AV-17, BDE-10P, and AN-2F, J. Appl. Chem. USSR 38, 1222 (1965); [Trans1. of 2h. Prikl. KhIm. 38, 1235 (1965)]; Chem. Abstr. 63, 6360 (1965). 
302. V. S. Pakholkov and V. D. 01 'khin, Sorption of Chromtum(III) from Fluoride-Containing Solutions by Anion Resins AV-17, EDE-10P, and AN-2F, Izv. Vyssh. Uchebn. Zaved., Khım. Khim. Tekhno1. 9, 401 (1966), (Russian); Chem. Abstr. 66, 14321 (1967).

303. V. S. Pakholkov and V. E. Pantkarovskikh, Sorption of Chromium(III) from Fluoride-Containing Solutions by Anion Resins AV-17, EDE-10P, and AN-2F, Izv: Vyssh. Uchebn. Zaved., KhIm. Khim. Tekhno1. 9, 401 (1966), (Russian); Chem. Abstr. 66, 14321 (1967).

304. V. S. Pakholkov and V. E. Panikarovskikh, Sorption of Sexavalent Chromium from Aqueous Solutions Containing Chromium Trioxide and Hydrofluoric Acid, or Chromium Trioxide, Hydrofluorlc Acld and Hydrochloric (or Sulphuric) Ac1d, Izv. Vyssh. Uchebn. Zaved., Kh1m. KhIm. Tekhno1. 9, 898 (1966), (Russian); Anal. Abstr. 15, 151 (1968); Chem. Abstr. 66, 108615 (1967).

305. V. S. Pakholkov and V. E. Panikarovskikh, Sorption of Molybdenum from Hydrofluoric Acld Solutions by Anion Exchangers, Izv. Vyssh. Uchebn. Zayed., Kh1m. Khim. Tekhno1. 10, 53 (1967), (Russian); Chem. Abstr. 67, 15246 (1967).

306. V. S. Pakholkov and V. E. Panikarovskikh, Anion Exchange Separation of Chromium and Molybdenum from Tungsten in Hydrofluoric Acld Solution, Izv. Vyssh. Uchebn. Zaved., Khim. Khim. Tekhno1. 10, 168 (1967), (Russian); Anal. Abstr. 15, 3309 (1968); Chem. Abstr. 67, 50044 (1967); Nuc1. Sc1. Abstr. 22, $48 \overline{902}$ (1968).

307. V. S. Pakholkov and V. V. Rylov, Sorption by Anton Exchangers AV-17, EDE-10P, and AN-2F of Iron(III) from Solution Containing Fluoride, Izv. Vyssh. Uchebn. Zaved., Tsvetn. Metall. 6, 114 (1963), (Russian); Chem. Abstr. 60, 15182 (1964).

308. V. S. Pakholkov and V. V. Rylov, Sorption of Beryllium and Aluminum from Hydrochlorlc Actd-Hydrofluorlc AcId and Sulfurlc Acld-Hydrofluorlc Acld Solutions by the AV-17, EDE-10P, and AN-1F Anton Exchangers, Tr. Ural. Polftekh. Inst. No. 148, 68 (1966), (Russ1an); Chem. Abstr. 68, 99020 (1966).

309. V. S. Pakholkov and S. E. Simakov, Separation of Vanadium and Urantum In $\mathrm{H}_{2} \mathrm{SO}_{4}$-HF Solutions by Neans of the Anton Exchangers AV-17, EDE-10P, and Al-2F, J. Appl. Chem. USSR 37, 2536 (1964); [Trans1. of Zh. Pr1k1. Kh1m. 37, 2565 (1964)]; Chem. Abstr. 62, 9757 (1965); Nucl. Sc1. Abstr. 19, $11 \overline{297}$ (1965).

310. V. S. Pakholkov and S. E. Sinakov, Sorption of Vanadium from the Solutions of $\mathrm{H}_{2} \mathrm{SO}_{4}-\mathrm{HF}$ wth the Anton Exchangers AV-17, $\mathrm{BDE}-10 \mathrm{P}$, and $\mathrm{AN}-2 \mathrm{~F}$, IzV. Vyseh. Uchebn. Zaved., Tovetn. Ketall. 7, 82 (1964), (Ruse1an); Anal. Abstr. 13, 1239 (1966); Chem. Abstr. 62, 7150 (1966).

311. V. S. Pakholkov and S. E. S1makov, Sorption of Mloblum and Tantalum from Sulfurlc Acid-Hydrofluortc Acld solutions by keans of the Anton Exchangers AV-17, GDE-10P, and AN-2E, Izv. Vyash. Uchabn. Zaved., Tevetn. Meta11. 8, 102 (1965), (Bussian); Ana1. Abstr. 13, 4796 (1966); Chem. Abstr. 63, 4980 (1965). 
312. V. S. Pakholkov and S. E. Simakov, Anfonic Separation of Niobium from Tantalum in HC1-HF Solutions with Ammonium Chloride, Izv. Vyssh. Uchebn. Zaved., Tsvetn. Metall. 9, 77 (1966), (Russian); Chem. Abstr. 65, 5084 (1966).

313. V. S. Pakholkov and V. F. Stikhin, The Sorption of Uranium(VI) from Hydrofluoric Acld Solutions on EDE-10P and AN-2F Weakly Basic AnionExchange Resins, AEC-Tr-6716, p. 183 (1967); [Trans1. of Cooprecipitation and Adsorption of Radioactive Elements, V. M. Vdovenko, Ed., MoscowLeningrad, p. 165 (1965)]; Nuc1. Sc1. Abstr. 19, 32314 (1965); Chem. Abstr. 63, 14088 (1965).

314. V. S. Pakholkov and A. S. Suntsov, Behavior of Ions of Certain Elements During Sorption from Hydrogen Fluoride, Ammontum Fluoride-Hydrogen Fluoride ( $\mathrm{HN}_{4} \mathrm{~F}-\mathrm{HF}$ ), and Ammonlum Fluoride Solutions by Anion Exchangers, Izv. Vyssh. Uchebn. Zaved., Tsvetn. Meta11. 18, 42 (1975), (Russian); Chem. Abstr. 83, 152860 (1975).

315. V. S. Pakholkov and A. S. Suntsov, Sorption of Niobium and Tantalum. from MF $5-\mathrm{HF}-\mathrm{H}_{2} \mathrm{O}$ on Anion-Exchange Resins and Amphoteric-Exchange Resins, J. Appl. Chem. USSR 49, 773 (1976); [Transl. of Zh. Prik1. Khim. 49, 737 (1976)]; Chem. Abstr. 85, 10651 (1976).

316. V. S. Pakholkov and A. S. Suntsov, Sorption of Elements of the Titanium Subgroup from $\mathrm{MF}_{4}-\mathrm{HF}-\mathrm{H}_{2} \mathrm{O}$ Solutions by Anion-Exchange Resins and AmphotericExchange Resins, J. App1. Chem. USSR 49, 776 (1976); [Transl. of Zh. Prik1. KhIm. 49, 740 (1976)]; Chem. Abstr. 85, 10652 (1976).

317. V. S. Pakholkov, A. S. Suntsov, and V. N. Rychkov, Ion Exchange on AnionExchange Resins and Ampholites in Solutions of $\mathrm{UO}_{2} \mathrm{~F}_{2}-\mathrm{HF}$ (NH4 $\mathrm{FHF}_{2} \mathrm{NH}_{4} \mathrm{~F}$ ) $-\mathrm{H}_{2} \mathrm{O}$ In Processes of Sorption and Desorption, Sov, Radiochem. 19, 655 (1977); Trans1. of Radiokhim. 19, 787 (1977).

318. V. S. Pakholkov, V. L. Zabolotnykh, and V. S. Ustinov, Sorption by an Anion Exchanger of Titanium and Zirconium from Fluoride Solutions, Izv. Vyssh. Uchebn. Zaved., Tsvetn. Meta11. 8, 93 (1965), (Russ1an); Chem. Abstr. 64, 1384 (1966).

319. E. S. Pal'shin, B. F. Myasoedov, and A. V. Davydov, Analytical Chemistry of Protactinium, (Trans1. from Russ. by J. Schmorak), Ann Arbor-Humphrey Pub., Ann Arbor, (1970).

320. N. A. Parpiev and I. A. Maslennikov, Ion-Exchange Study of Fluoride Complexes of Silicon and Germanlum, Uzb. Khim. Zh. 12, 6 (1968), (Russian); Chem. Abstr. 69, 80954 (1968).

321. G. Phillips and E. N. Jenkins, The Removal of Plutonlum Before the Analysis of Mlxed F1ssion Products, J. Inorg. Nucl. Chem. 4, 220 (1957); Nucl. Sc1. Abstr. 11, 8877 (1957).

322. G. PInte, Application of Neutron Activation Analysis to the Study of Impurities in Yolybdenum, Tungsten and Nuclear Graphite, CEA-R 3267 (1967), (French); Nuc1. Sc1. Abstr. 22, 16387 (1968). 
323. N. R. Piper, Radiochemical Purification of Microgram and Sub-Microgram Amounts of Beryllium, Anal. ChIm. Acta 42, 423 (1968); Nucl. Sc1. Abstr. $\underline{22}, 45153$ (1968).

324. P. M. Plaisance and R. Guillaumont, Fluoro- and Chlorofluoro-Complexes of Pentavalent Protactinium, Radiochim. Acta 12, 32 (1969), (French); Nucl. Sci. Abstr. 23, 40629 (1969).

325. E. Pluchet and R. Muxart, Chromatography of Tetravalent Protactinium in Hydrochloric Ac1d Solutions on Anton Exchange Resins, Bull. Soc. Chim. Fr., (2), 372 (1961), (French); Anal. Abstr. 8, 4589 (1961).

326. Pratt and Whitney Afrcraft Corp., Determination of Z1rconium in Columbium, Methods for the Analysis of Columbium and Its Alloys, PWAC-340, p. 43 (1961).

327. 0. E. Presnyakova, R. S. Prishchepo, and G. Ya. Avrutskaya, Separation of Beryllium and Aluminum in Fluoride - Containing Solutions on AV-17 Anion Exchange Resin In the Fluortde Form, S1b. Chem. J. (2), 209 (1969); [Trans1. of Izv. SIb. Otd. Akad. Nauk SSSR, Ser. Khim. Nauk (2), 102 (1969)]; Chem. Abstr. 71, 56250 (1969); Anal. Abstr. 19, 1057 (1970).

328. V. A. Pronin, E. N. Gilbert, and P. I. Artyukhin, Neutron-Activation Method for Determination of Impur1ties in Thallium, Izv, S1b. Otd. Akad. Nauk SSSR, Ser. KhIm. Nauk (1) 3, 88 (1966), (Russian); Chem. Abstr. 65, 9719 (1966); Nucl. Sc1. Abstr. 20, 45596 (1966).

329. S. Przeszlakowsk1, Chromatography of Some Ions on Paper Impregnated with Liquid Anton-Exchangers Using Hydrofluoric Acid Solutions as the Moblle Phase, Chem. Anal. 12, 321 (1967), (Pol1sh); Anal. Abstr. 15, 3761 (1968); Chem. Abstr. 68, $26 \overline{505}$ (1968).

330. K. S. Rajan and J. Gupta, Separation of Zircontum and Hafnium by Means of Anton-Exchange Resins. I. Qualitative Studies, J. Sc1. Ind. Res. (India) B 14, 453 (1955); Chem. Abstr. 50, 5439 (1956).

331. K. S. Rajan and J. Gupta, Separation of Z1rconium and Hafnium Using Anton-Exchange Resins. II. Influence of Physical Factors, J. Sc1. Ind. Res. (Ind1a) 16B, 459 (1957); Chem. Abstr. 52, 5189 (1958).

332. G. N. Razumova and I. D. Shuba, Determination of Rhenium in Germanium by the Neutron Activation Method, Sov. Radiochem. 11, 345 (1969); [Trans1. of Radiokh1m. 11, 354 (1969)]; Chem. Abstr. 71, $77 \overline{006}$ (1969); Anal. Abstr. 19, $216 \overline{7}$ (1970).

333. G. N. Razumova, I. D. Shuba, and I. Ya. Vasil' ev, Neutron Act1vation Determination of Trace Impurities in Speclal Purity Germanium, Sov. Radiochem. 12, 116 (1970); [Trans1. of Radiokh1m. 12, 133 (1970)]; Nuc1. Sc1. Abstr. 24,38789 (1970).

334. J. C. Rtcq, Use of Superimposed Ion Exchange Columns in Activation Analyg18, J. Radloanal. Chem. 1, 443 (1968), (French); Chem. Abstr. 70, 25382 (1969); Nucl. Sc1. Abstr. 23, 11472 (1969). 
335. G. RIzzardi and N. Bertazzi, Separation and Identification of Some Flssion Products of Thorium by Ion-Exchange Chromatography, Ric. Sci. Riv. 36(8), 699 (1966), (Italian); Anal. Abstr. 14, 7405 (1967).

336. E. Rona, L. Muse, and B. L. Brandau, Protactinium in Deep Sea Sediments, Physico-Chemle du Protactinium, Proc. Orsay Conf., 1965, Centre National de la Rech. Sci., Paris, p. 133 (1966).

337. J. C. Rouchaud, M. Fedoroff, and G. Revel, Determinarion of Silicon in Metals by Thermal Neutron Activation, J. Radioanal. Chem. 38, 185 (1977).

338. N. P. Rudenko and 0. M. Kalinkina, The Chromatographic Method of Separating Radioactive Hafnium and Tantalum, Russ. J. Inorg. Chem. 2, 389 (1957); [Transl. of Zh. Neorg. Khim. 2, 959 (1957)]; AEC-Tr-4054; Nucl. Sci. Abstr. 15, 14355 (1961); Chem. Abstr. 52, 915 (1958).

339. D. I. Ryabchikov and G. E. Kuril'chikova, Determination of Small Amounts of Boron in the Presence of Fluorlne and Silicon, J. Anal. Chem. USSR 19, 1389 (1964); [Transl. of Zh. Anal. Khim. 19, 1495 (1964)]; Chem. Abstr. 62, 9774 (1965).

340. J. L. Ryan and E. J. Wheelwright, The Recovery, Purification, and Concentration of Plutonium by Anion Exchange in Nitric Acid, U.S. AEC Rept. HW-55893 (1959); Nucl. Sc1. Abstr. 13, 22147 (1959).

341. E. Sabbion1, E. Marafante, L. Goetz, and C. Birattar1, Cyclotron Production of Carrier Free ${ }^{48} \mathrm{~V}$ and ${ }^{48} \mathrm{~V}$ Compounds for Metabolic Studies In Rats, Radiochem. Radioana1. Lett. 31, 39 (1977).

342. M. Salutsky, K. Shaver, A. Elmlinger, and M. L. Curt1s, Separation of Protactinium from Uranium Residues, J. Inorg. Nuc1. Chem. $\underline{3}, 289$ (1956).

343. 0. Samuelson, Ion Exchange Separations in Analytical Chemistry, W1ley, New York (1963).

344. T. Sawada and S. Kato, Method for the Determinations of N1oblum and Tantalum in Titanfum Alloys and Zirconium-Base Alloys, Nippon Kinzoku Gakka1shi 28, 180 (1964), (Japanese); Nuc1. Sc1. Abstr. 18, 35478 (1964).

345. U. Schindewolf and J. W. Irvine, Jr., Preparation of Carrier-Free Vanadium, Scandium, and Arsenic Activities from Cyclotron Targets by Ion Exchange, Anal. Chem. 20, 906 (1958).

346. H. Schmled and G. Ziffermayer, Activation Analysis as an Analytical Method In Manufacture of Valves (Electron Tubes), Mcrotecnic 22(4), 293 (1968); Chem. Abstr. 70, 63944 (1969).

347. W. W. Schulz, Macroreticular Ion Exchange Resin Cleanup of Purex Process TBP Solvent, ARH-SA-58-RD (1970); Nuc1. Sc1. Abstr. 25, 34679 (1971).

348. W. W. Schulz and G. E. Benedict, Neptuntum-237, Product Ion and Recovery, AEC Critical Review Sertes, USAEC, Pp. 45-51 (1972); Nucl. Sc1. Abstr. 27, 219 (1973) (TID-25955). 
349. H. Seltner, W. Kies1, F. Kluger, and F. Hecht, Wet-Chemical Analysis and Determination of Trace Elements by Neutron Activation In Meteorites, J. Radioanal. Chem. 7, 235 (1971).

350. M. P. Semov, Sorption of Boron from Hydrofluoric Acid Solutions by AV-17 Anion Exchange Resin and Its Use in Analysis of Pure Materials, J. Anal. Chem. USSR 23, 200 (1968); [Trans1. of Zh. Anal. Khim. 23, 245 (1968)]; Chem. Abstr. 68, 119051 (1968).

351. M. M. Senyavin, Ion Exchange and Ion-Exchange Chromatography in the Analytical Chemistry and Technology of Inorganic Substances, Ionny1 Obmen 1 Ego Primenenie, Akad. Nauk SSSR., Dtdel. KhIm. Nauk, K. V. Chmutov, Ed., Moscow, p. 84 (1959), (Russian); Chem. Abstr. 54, 12426c (1960).

352. M. K. Sherief and F. Grass, Sep ation of Plutonium-239(IV) from Zirconium-95, Niobium-95, Ruther .um-106 and Rhodium-106 by Anion-Exchange Resin from Mixed Methanol-Nitric Acid Solution, Atomkernenergie 17, 124 (1971); Anal. Abstr. 22, 2192 (1972).

353. B. I. Shramban and V. I. Ksenzenko, The Stability of the AV-17 $\times 8$ AnionExchange Resin in Hydrofluoric Ac1d, Russ. J. Phys. Chem. 51, 853 (1977); [Transl. of Zh. F1z. Khim. 51, 1454 (1977)].

354. W. B. Silker, The Radiochemical Determination of Plutonium in Soil, Hanford Radiological Sctentific Research and Development Annual Report for 1964, Radiological chem. BWNL-36, p. 3.37 (1965); Nuc1. Sc1. Abstr. 20, 8980 (1966).

355. W. B. Silker, A Rapid Method for Determination of Plutonium in Urine, Health Phys. 11, 965 (1965); Nucl. Sc1. Abstr. 19, 38555 (1965); BWNL-36 III, p. 3.39 (1965).

356. M. Span and J. Span, Direct Sequential Separation of Zircontum, Niobium, Cobalt, Iron, and Zinc by Anion Exchange, Vestn. Slov. Kem. Drus, p. 14 (Russian), P. 15 (English), (1968); Chem. Abstr. 71, 27114 (1969); Nuc1. Sc1. Abstr. 23, 11631 (1969).

357. J. D. Smith, Spectrophotometric Determination of Traces of Tin In Rocks, Sediments and Soils, Anal. Chim. Acta 57, 371 (1971).

358. M. Simkova and V. P1nkas, Determination of Impurities in Silicon by Act Ivation Analysis, Isotopenpraxis 3, 88 (1967), (German); Nuc1. Sc1. Abstr. 21, 30369 (1967); Chem. Abstr. 71, 9339 (1969).

359. S. Spauszus and M. HeImer, Direct Determination of Nloblum and Tantalum with the Antonic Exchanger Wofat1t SBW, Chem. Tech. (Ber11n) 13, 96 (1961), (German); Anal. Abstr. 8, 4140 (1961); Nucl. Sc1. Abstr. 15, 15568 (1961).

360. S. Specht, Concerning Some Research Work on Transuranium Elements at the Institute of Radiochentstry in Munich, Proc. Symp. on Transuranium Element8, Llege, 1969, p. 213 (German). 
361. G. L. Starobinets and I. F. Gleim, Ion Exchange of Weak Organic Electrolytes, Russ. J. Phys. Chem. 39, 1166 (1965); [Trans1. of Zh. Fiz. KhIm. 39, 2188 (1965)].

362. L. Stein, Protactinium Fluorides, Physico-Chemie du Protactinium, Proc. Orsay Conf. 1965, Centre National de la Rech. Sci., Par1s, p. 101 (1966).

363. E. P. Steinberg, The Radiochemistry of Niobium and Tantalum, NAS-NS-3039 (1961), Available from the Office of Technical Services, Dept. of Commerce, Wash., D.C.

364. E. Stelnnes, Determination of S1xteen Trace Elements in H1gh-Purity Iron by Neutron Activation and Gamma-Ray Spectrometry with a Lithium-Doped Germantum Detector, Scand. J. Metall. 1, 137 (1972); Chem. Abstr. 77, 56140 (1972); Anal. Abstr. 23, 3862 (1972).

365. M. Stoeppler and K. May, Plutonium Decontamination During Uranium Separatiol rom Fission Products, Radiochem. Radioanal. Iett. 33, 413 (1978).

366. D. P. Stricos, A. Procedure for the Quantitative Recovery of Pa-233 from Irradlated Thorla, KAPL-M-6554 (1966); Anal. Abstr. 14, 4614 (1967); Nuc1. Sci. Abstr. 20, 29331 (1966).

367. T. S. Studenskaya, N. D. Fedorova, V. V. Stepin, and V. L. Zolotavin, Chromatographic Separation of Vanadium, Molybdenum and Tungsten from Fluoride Solution, Tr. Vses. Nauchno-Issled. Inst. Stand. Obraztsov Spektr. Etalonov I, 22 (1964), (Russian) Anal. Abstr. 13, 3393 (1966).

368. K. F. Sugawara, Spectrophotometric Determination of Trace Amounts of Zirconlum, Titanium, and Molybdenum In Tungsten Using Arion Exchange Separations, Anal. Chem. 36, 1373 (1964); ASD-TDR-63-358 (1963).

369. K. F. Sugawara, Determination of Trace Amounts of Molybdenum and Niobium in Tungsten Using a Small Anlon Exchange Column, ML-TDR-64-268 (AD-608074) (1964); Nucl. Sc1. Abstr. 19, 28217 (1965).

370. K. F. Sugawara, Quantitative Chemical Analysis of Sma11 Surface Areas of Tungeten Metal for Zirconlum and Titanlum, Abstr. of Papers, 152nd. ACS MeetIng, New York, Sept. 1966, p. B-93.

371. D. C. Sutton, Determination of Beryllium in Urine by Ion Exchange Separation and Fluorimetric Measurement, HASL-134 (1963); Nuc1. Sc1. Abstr. 17, 25114 (1963).

372. S. Suzuk1 and Y. Inque, The Chemistry of Protactinium. I. The Preparation of Protactinium-233 and the Purification of Protactinium-231, Bul1. Chem. Soc. Jpn. 39, 490 (1966); Nucl. Sc1. Abstr. 20, 29335 (1966).

373. T. Suzuk1, K. Toutsum1, and N. Touj1, Determination of Sma11-Amounts of Stlicon in Zr-Nb Alloys by Anton Exchange Separation - Molybdenum Blue Photometry, Power Reactor and Nucl. Fuel Dev. Corp. Tokal Works, Japan, Prog. Rept. PNCT-831-74-01, p. 59 (1974); ERDA Energy Res. Abstr. 1 , 26191 (1976). 
374. M. Szlaurova, v. Koprda, and M. Fojtik, Arion-Exchange in Mixed Solvent Systems. V. Separation Scheme for Fifteen Radionuclides by Ion-Exchange, J. Radioanal. Chem. 21, 211 (1974); Nucl. Sc1. Abstr. 30, 31553 (1974).

375. K. Tada and H. Horiguch1, Determination of Cobalt, Titanium and Tantalum in Cemented Carbides; Separations by Anion-Exchange, Bunseki Kagaku (Japan Analyst) 12, 799 (1963), (Japanese); Anal. Abstr. 12, 595 (1965); Nuc1. Sci. Abstr. 18, 39090 (1964); Chem. Abstr. 60, 4758 (1964).

376. G. M. Thompson, R. L. Walker, J. A. Carter, and D. N. Lumsden, Procedures for Thorium and Uranium Extraction from Calcite, Radiochem. Radioanal. Lett. 16, 53 (1973); Anal. Abstr. 27, 655 (1974).

377. R. C. Thompson, Q. Van Winkie, and J. G. Malm, Extraction of Protactinium from Carbonate-Insoluble Residues of Uranium Ores. III. Manganese Dloxide Procedure, Production and Separation of U-233. Collected Papers, L. I. Katzin, Ed., TID-5223 (Pt. 1), Paper 6.4, p. 276 (1952).

378. 0. K. Tikhonova, Z. I. Otmakhova, and 0. V. Chashchina, Chemicospectrographic Analysis of High-Purity Gallium and Gallium Arsentde, J. Anal. Chem. USSR 28, 1145 (1972); [Trans1. of Zh. Anal. Khim. 28, 1288 (1972)]; Chem. Abstr. 79, 111388 (1973).

379. C. H. Toy and R. T. Van Santen, Ion Exchange Separation and Determination of Silicon in Silver-Infiltrated Tungsten, Anal. Chem. 36, 151 (1964).

380. U.S. Atomic Energy Comm., Determination of Plutonium in Various Matrices, Quick Methods for Radiochemical Analysis, Tech. Rept. Series No. 95, IAEA, Vlenna, p. 41 (196?); STI/DOC/10/95.

381. P. Van den Winke1, A. Speecke, and J. J. Hoste, Separation Scheme for the Determination of Nine Elements in Biological Materlal, Proc. Symp. on Nuclear Activation Techniques in the Life Sciences, Amsterdam, May 1967, STI/PUB/55, IAEA, Vienna, p. 159 (1967); Nucl. Sc1. Abstr. 23, 4544 (1969).

382. L. P. Varga and H. Freund, The Formation Constants of the Tantalum Fluoride System. I. Potentiometric and Anton Exchange Studies - Evidence for Species of Coordination Number Nine, J. Phys. Chem. 66, 21 (1962); Chem. Abstr. 56, 9484 (1962).

383. J. A. Velandia and A. K. Perkons, An Ion-Exchange Group-Separation Scheme for Rapid Analysis of the Components of Neutron-Activated Biological Tissues, J. Radioanal. Chem. 20, 473 (1974); Nuc1. Sc1. Abstr. 30, 20601 (1974).

384. I. V. Vinarov and N. S. Shulgina, Sorption and Separation of Zirconium and Hafnium on Native Antonttes, Ukr. Khim. Zh. 31, 1219 (1965), (Russian); Nuc1. Sc1. Abstr. 20, 29352 (1966).

385. 0. A. Vita, C. R. Walker, C. F. Trivisonno, and R. W. Sparks, AnionExchange Separation for the Determination of Uranium in Complex Solutions, Anal. Chem. 42, 465 (1970); Anal. Abstr. 20, 2376 (1971). 
386. A. F. Voigt, G. I. Jewett, E. C. Jacobson, K. L. Malaby, and J. D. Woods, The Determination of Trace Amounts of Tantalum and Tungsten in Metals, Proc. Conf. on Modern Trends In Activation Analysis, College Sta. Texas, 1965, p. 26.

387. A. I. Vulikh, M. K. Zagorskaya, and V. I. Ksenzenko, Absorptton of Hydrogen Fluoride from the Gas Phase by Anion Exchangers, Dok1. Akad. Nauk SSSR 175, 1059 (1967), (Rus8ian); Chem. Abstr. 68, 53616 (1968).

388. R. I. Walter, Anton Exchange Studies of Sc(III) and V(IV). Separation of Scandium, Titanium and Vanadium, J. Inorg. Nucl. Chem. 6, 58 (1958); Nucl. Sc1. Abstr. 12, 9752 (1958).

389. J. H. Watkinson, Spectrometric Determination of Molybdenum, Tungsten and Vanadium in Solls and Plants. I. In Solls, N.Z. J. Sci. 1, 201 (1958); Ana1. Abstr. 6, 1134 (1959).

390. M. R. Weiler and W. Y. Matsumoto, Determination of U-232 and U-233 in Irradlated Thorlum, BNWL-159 (1965); Nucl. Sc1. Abstr. 20, 8818 (1966).

391. H. V. Weiss and W. H. Shipman, Radiochemical Determination of Plutonium in Urine, Anal. Chem. 33, 37 (1961); USNRDL-TR-450 (1960).

392. G. A. Welford, W. R. Collins, Jr., R. A. Morse, and D. C. Sutton, The Sequentlal Analysis of Long Range Fallout Debris, Talanta 5,168 (1960); Chem. Abstr. 55, 8076 (1961).

393. C. U. Wetlesen, Analysis of Alloys of Titanium, Nlobium and Tantalum by Ion Exchange, Anal. Chim. Acta 22, 189 (1960); Nucl. Sci. Abstr. 14, 24076 (1960).

394. R. M. Wheaton and W. C. Bauman, Properties of Strongly Basic Anion Exchange Resins, Ind: and Eng. Chem. 43, 1088 (1951); Chem. Abstr. 45, 7726 (1951).

395. R. Wickbold, The Enrtchment of Very Small Amounts of Silica by Ion Exchange, Z. Anal. Chem. 171, 81 (1959), (German); Anal. Abstr. 7, 2662 (1960).

396. D. H. W11k1ns, The Separation and Determination of N1ckel, Chromium, Cobalt, Iron, Titantum, Tungsten, Molybdenum, Niobium and Tantalum In a High Temperature Alloy by Anlon-Exchange, Talanta 2, 355 (1959); Chem. Abstr. 54, 7429 (1960).

397. A. I. Williams, Determination of Niobium In Metals and Alloys, Analyst 92, 43 (1967); Nuc1. Sc1. Abstr. 22, 22971 (1968); Anal. Abstr. 15, 2592 (1968).

398. L. W1sh, Quantitative Ion-Exchange Separations for Sequential Radiochemical Analys18, U.S. Naval Radiological Defense Lab. Rept. USNRIL-TR-185 (1957); Nuc1. Sc1. Abstr. 12, 2767 (1958). 
399. L. Wish, Quantitative Radiochemical Analysis by Ion Exchange. IV. Uranium and Tellurium, U.S. Naval Radiological Defense Lab. Rept. USNRDL-TR-312 (1959); Nucl. Sc1. Abstr. 13, 12479 (1959).

400. L. Wish, Anion Exchange Behavior in Mixed Acid Solutions and Development of a Sequential Separation Scheme, Anal. Chem. 31, 326 (1959).

401. L. Wish and M. Rowe11, Sequential Analysis of Tracer Amounts of Np, U and Pu in Fission-Product Mixtures by Anion Exchange, U.S. Naval Radlological Defense Lab. Rept. USNRDL-TR-117 (1956); Nucl. Sc1. Abstr. 11, 3702 (1957).

402. J. D. Wolszen, J. R. Hayes, and W. H. Hill, Applications of Anion Exchange Resins to Determination of Boron, Anal. Chem. 29, 829 (1957).

403. P. H. Woods and L. D. Cockere11, Anion Exchange of Titanium (IV) in Hydrofluoric Acid, J. Am. Chem. Soc. 80, 1534 (1958).

404. Jeng-Tsong Yang, The Separation of Protactinium from Tantalum by Ion Exchange, Compt. Rend. 231, 1059 (1950); Chem. Abstr. 45, 3730 (1951); Nucl. Sci. Abstr. $\underline{5}, 18 \overline{26}$ (1951). 